\title{
The Graham-Knuth-Patashnik recurrence: Symmetries and continued fractions
}

\author{
Jesús Salas \\ Departamento de Matemáticas \\ Universidad Carlos III de Madrid \\ Avda. de la Universidad, 30 \\ 28911 Leganés, Spain \\ Grupo de Teorías de Campos y \\ Física Estadística \\ Associate Unit UC3M-IEM (CSIC), Spain \\ jsalas@math.uc3m.es \\ Alan D. Sokal \\ Department of Mathematics \\ University College London \\ Gower Street \\ London WC1E 6BT \\ United Kingdom \\ sokal@math.ucl.ac.uk \\ Department of Physics \\ New York University \\ 726 Broadway \\ New York, NY 10003, U.S.A. \\ sokal@nyu.edu
}

Submitted: Aug 7, 2020; Accepted: Apr 1, 2021; Published: May 7, 2021

(C) The authors. Released under the CC BY-ND license (International 4.0).

\begin{abstract}
We study the triangular array defined by the Graham-Knuth-Patashnik recurrence

$$
T(n, k)=(\alpha n+\beta k+\gamma) T(n-1, k)+\left(\alpha^{\prime} n+\beta^{\prime} k+\gamma^{\prime}\right) T(n-1, k-1)
$$

with initial condition $T(0, k)=\delta_{k 0}$ and parameters $\boldsymbol{\mu}=\left(\alpha, \beta, \gamma, \alpha^{\prime}, \beta^{\prime}, \gamma^{\prime}\right)$. We show that the family of arrays $T(\boldsymbol{\mu})$ is invariant under a 48-element discrete group isomorphic to $S_{3} \times D_{4}$. Our main result is to determine all parameter sets $\boldsymbol{\mu} \in \mathbb{C}^{6}$ for which the ordinary generating function $f(x, t)=\sum_{n, k=0}^{\infty} T(n, k) x^{k} t^{n}$ is given by a Stieltjes-type continued fraction in $t$ with coefficients that are polynomials in $x$. We also exhibit some special cases in which $f(x, t)$ is given by a Thron-type or Jacobi-type continued fraction in $t$ with coefficients that are polynomials in $x$.
\end{abstract}

Mathematics Subject Classifications: 05A10 (Primary); 05A15, 05A19, 30B70 (Secondary) 


\section{Contents}

1 Introduction 3

2 Symmetries of the GKP recurrence $\quad 6$

2.1 Some special symmetries . . . . . . . . . . . . . . . . . 6

2.2 Determination of the symmetry group . . . . . . . . . . . . . . . . . 9

2.3 Further scaling properties of the GKP recurrence . . . . . . . . . . . 12

3 Characterization of the GKP recurrences with an S-fraction representation 14

3.1 Computer-assisted search . . . . . . . . . . . . . . . . . . 15

3.2 A "master S-fraction" for permutations . . . . . . . . . . . . . 20

3.3 Proof that the candidate families have an S-fraction representation . . . . . 26

3.3.1 Families $1 \mathrm{a}$ and $1 \mathrm{~b} \ldots \ldots \ldots . \ldots . \ldots 26$

3.3 .2 Families $2 \mathrm{a}$ and $2 \mathrm{~b} \ldots \ldots \ldots \ldots . \ldots \ldots 27$

3.3.3 Families $3 \mathrm{a}$ and $3 \mathrm{~b} \ldots \ldots \ldots \ldots$

3.3.4 Families $4 \mathrm{a}$ and $4 \mathrm{~b} \ldots \ldots \ldots \ldots . \ldots . \ldots . \ldots . \ldots 31$

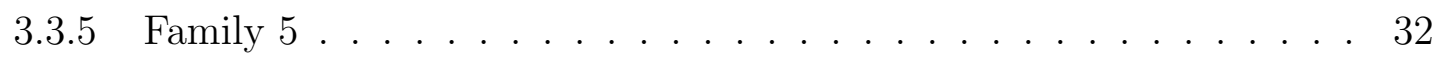

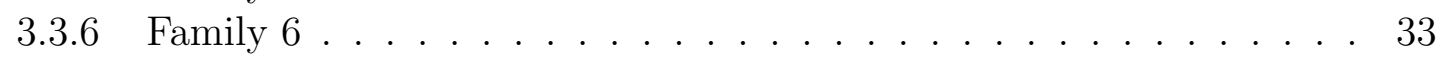

4 Preliminaries for T-fractions and J-fractions $\quad 34$

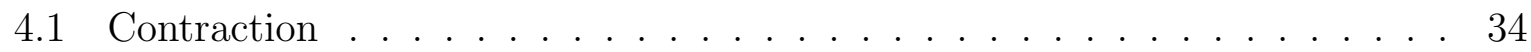

4.2 Binomial transform . . . . . . . . . . . . . . . . . . . . . 35

5 Some GKP recurrences with a T-fraction or J-fraction representation 39

5.1 Examples of GKP recurrences with T-fraction or J-fraction arising from the binomial transform . . . . . . . . . . . . . . . . . . 39

5.1 .1 Families $7 \mathrm{a}$ and $7 \mathrm{~b} \ldots \ldots \ldots . \ldots . \ldots 39$

5.2 Examples of GKP recurrences with T-fraction not arising from the binomial transform . . . . . . . . . . . . . . . . . . . . 41

5.2.1 Families 8a and 8b: Generalized Ward polynomials and their dual . 41

5.2.2 Families 9a and 9b: Conjectured T- and J-fractions . . . . . . . . . 43

5.3 Examples of GKP recurrences with J-fraction not arising from the binomial

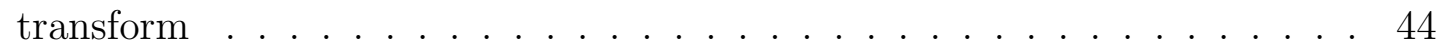

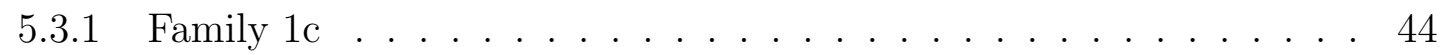

6 Open questions $\quad 44$

6.1 T-fractions, J-fractions, and binomial transforms . . . . . . . . . . . . . . 44

6.2 Coefficientwise Hankel-total positivity . . . . . . . . . . . . . . . 46

6.3 A generalization: The Graham-Knuth-Patashnik-Zhu recurrence . . . . . 50 
A Matrix product of two GKP arrays

A.1 General results . . . . . . . . . . . . . . . . . . . 52

A.2 Special matrix on the left . . . . . . . . . . . . . 57

A.3 Special matrix on the right . . . . . . . . . . . . . . . . . 62

B Inverse pairs of lower-triangular arrays $\quad 66$

\section{Introduction}

Graham, Knuth and Patashnik (GKP), in their book Concrete Mathematics [34], posed the following "research problem" [34, Problem 6.94, pp. 319 and 564]:

Problem 1.1. Develop a general theory of the solutions to the recurrence

$$
T(n, k)=(\alpha n+\beta k+\gamma) T(n-1, k)+\left(\alpha^{\prime} n+\beta^{\prime} k+\gamma^{\prime}\right) T(n-1, k-1)
$$

for $n \geqslant 1$ and $k \in \mathbb{Z}$, with initial condition $T(0, k)=\delta_{k 0}$. (Here and in the following, $\delta_{a b}$ denotes the Kronecker delta.)

By induction on $n$ we clearly have $T(n, k)=0$ if $k<0$ or $k>n$. Therefore, for each choice of the parameters $\boldsymbol{\mu}=\left(\alpha, \beta, \gamma, \alpha^{\prime}, \beta^{\prime}, \gamma^{\prime}\right)$, we obtain a unique solution $T(n, k)=$ $T(n, k ; \boldsymbol{\mu})$, forming a triangular array $\boldsymbol{T}(\boldsymbol{\mu})=(T(n, k ; \boldsymbol{\mu}))_{0 \leqslant k \leqslant n}$. Here the parameters $\boldsymbol{\mu}$ can be considered to be indeterminates, in which case the matrix elements $T(n, k ; \boldsymbol{\mu})$ belong to the polynomial ring $\mathbb{Z}[\boldsymbol{\mu}]$; or they can be real or complex numbers, in which case the matrix elements $T(n, k ; \boldsymbol{\mu})$ are likewise real or complex numbers. We shall take each of these two points of view at appropriate places in this paper.

Given a triangular array $\boldsymbol{T}=(T(n, k))_{0 \leqslant k \leqslant n}$, we define its row-generating polynomials

$$
P_{n}(x) \stackrel{\text { def }}{=} \sum_{k=0}^{n} T(n, k) x^{k},
$$

its ordinary generating function (ogf)

$$
f(x, t) \stackrel{\text { def }}{=} \sum_{n \geqslant 0} P_{n}(x) t^{n}=\sum_{n, k \geqslant 0} T(n, k) x^{k} t^{n},
$$

and its exponential generating function (egf)

$$
F(x, t) \stackrel{\text { def }}{=} \sum_{n \geqslant 0} P_{n}(x) \frac{t^{n}}{n !}=\sum_{n, k \geqslant 0} T(n, k) x^{k} \frac{t^{n}}{n !} .
$$

It is straightforward to check that the row-generating polynomials $P_{n}(x)=P_{n}(x ; \boldsymbol{\mu})$ of the GKP recurrence satisfy the linear differential recurrence

$$
P_{n}(x)=\left[n\left(\alpha+\alpha^{\prime} x\right)+\gamma+\left(\beta^{\prime}+\gamma^{\prime}\right) x\right] P_{n-1}(x)+x\left(\beta+\beta^{\prime} x\right) \frac{d P_{n-1}(x)}{d x}
$$


for $n \geqslant 1$, with initial condition $P_{0}(x)=1$. Similarly, the exponential generating function $F(x, t)=F(x, t ; \boldsymbol{\mu})$ satisfies the first-order linear partial differential equation

$$
\left(1-\alpha t-\alpha^{\prime} x t\right) F_{t}=\left(\beta x+\beta^{\prime} x^{2}\right) F_{x}+\left(\alpha+\gamma+\left(\alpha^{\prime}+\beta^{\prime}+\gamma^{\prime}\right) x\right) F
$$

with initial condition $F(x, 0)=1$.

Explicit solutions for the matrix elements $T(n, k)$ or the egf $F(x, t)$ were found for some special cases of the parameters $\boldsymbol{\mu}$ by Théorêt [57-59], Neuwirth [40], Spivey [52], and Mansour and Shattuck [39]. In particular, Neuwirth [40] solved the case $\alpha^{\prime}=0$, while Spivey [52] solved three additional cases: (S1) $\beta=-\alpha$, (S2) $\beta=\beta^{\prime}=0$, and (S3) $\alpha / \beta=\alpha^{\prime} / \beta^{\prime}+1$. Wilf [64] pointed out that the PDE (1.6) can in principle be solved by the method of characteristics, and he showed how to obtain the solution (albeit in an unwieldy form) by using the MAPLE function pdsolve. Finally, Barbero, Salas and Villaseñor [2] explicitly solved the PDE (1.6) by the method of characteristics. In general this solution contains inverse functions [2, Theorems 2.1-2.4], but in many combinatorially interesting cases there exist closed-form expressions in terms of elementary functions [2, Appendix A]. It is worthy of note that the function $\boldsymbol{\mu} \mapsto \boldsymbol{T}(\boldsymbol{\mu})$ is not injective: there are some families of parameters $\boldsymbol{\mu}$ that produce the same triangular array $\boldsymbol{T}$ [57, section 2.4] [2, section 3]. Finally, some additional properties of the triangular arrays $\boldsymbol{T}=(T(n, k))_{0 \leqslant k \leqslant n}$ have recently been obtained by Spivey [54].

Our goal in the present paper is twofold: to study the symmetry group of the GKP recurrence, and to study some continued-fraction expansions of the ordinary generating function (1.3). Let us now discuss these two goals in turn.

By a "symmetry" of the GKP recurrence (1.1), we mean a map $M: \boldsymbol{\mu} \mapsto \boldsymbol{\mu}^{\prime}$ for which the array $\boldsymbol{T}\left(\boldsymbol{\mu}^{\prime}\right)$ can be written in a "simple" way in terms of $\boldsymbol{T}(\boldsymbol{\mu})$. (See Section 2 for more details of what we mean by "simple".) Here we will show that the symmetry group of the GKP recurrence is surprisingly large, and includes a 48-element discrete group that is isomorphic to $S_{3} \times D_{4}$.

It is well known that many combinatorial sequences $\boldsymbol{a}=\left(a_{n}\right)_{n \geqslant 0}$ with $a_{0}=1$ lead to an ogf that can be expressed as a continued fraction of Stieltjes type (or S-fraction for short),

$$
\sum_{n \geqslant 0} a_{n} t^{n}=\frac{1}{1-\frac{c_{1} t}{1-\frac{c_{2} t}{1-\cdots}}},
$$

for some coefficients $\boldsymbol{c}=\left(c_{i}\right)_{i \geqslant 1}$. (Both sides of this expressions are to be interpreted as formal power series in the indeterminate $t$.) This line of investigation goes back at least to Euler [23, 24], but it gained impetus following Flajolet's [27] seminal discovery that the S-fraction (1.7) can be interpreted combinatorially as a generating function for Dyck paths with a weight $c_{i}$ for each fall from height $i$. There are now literally dozens of sequences $\boldsymbol{a}=\left(a_{n}\right)_{n \geqslant 0}$ of combinatorial numbers or polynomials for which a continuedfraction expansion of the type (1.7) is explicitly known. 
In the generic S-fraction (1.7), the Taylor coefficients $a_{n}$ are polynomials in the Stieltjes coefficients $\boldsymbol{c}$ : these are the Stieltjes-Rogers polynomials $S_{n}\left(c_{1}, \ldots, c_{n}\right)$ [27]. If, however, one seeks conversely to express the Stieltjes coefficients $\boldsymbol{c}$ in terms of the Taylor coefficients $\boldsymbol{a}$, one obtains in general rational functions, not polynomials:

$$
\begin{aligned}
c_{1} & =a_{1} \\
c_{2} & =\frac{a_{2}-a_{1}^{2}}{a_{1}} \\
c_{3} & =\frac{a_{1} a_{3}-a_{2}^{2}}{a_{1}\left(a_{2}-a_{1}^{2}\right)} \\
& \vdots
\end{aligned}
$$

In particular, if one applies these formulae to the row-generating polynomials of the GKP recurrence, $a_{n}=P_{n}(x ; \boldsymbol{\mu})$, one obtains Stieltjes coefficients $c_{i}$ that, for $i \geqslant 2$, are rational functions of $x$ and the parameters $\boldsymbol{\mu}$. Nevertheless, for many specific cases of the GKP recurrence - including the binomial coefficients, the Stirling cycle and Stirling subset numbers, and the Eulerian numbers, among others - it is known that the Stieltjes coefficients $c_{i}$ are polynomials in $x$ (and fairly simple ones at that). Consequently, the second (and principal) goal of this paper is to obtain a complete determination of the submanifolds of $\boldsymbol{\mu} \in \mathbb{C}^{6}$ where the Stieltjes coefficients $\boldsymbol{c}$ are polynomials in $x$. These coefficients will always be of the form $c_{i}=c_{i 0}+c_{i 1} x$; and while a priori we allow $c_{i 0}$ and $c_{i 1}$ to be rational functions of the parameters $\boldsymbol{\mu}$, we will find a posteriori that they are in fact always polynomials in $\boldsymbol{\mu}$ (or more precisely, in suitable parameters coordinatizing the given submanifold). Our results are contained in Theorem 3.1 and Propositions 3.6-3.11.

More generally, we shall briefly consider some continued fractions of the Thron type (or T-fractions) [60],

$$
\sum_{n \geqslant 0} a_{n} t^{n}=\frac{1}{1-d_{1} t-\frac{c_{1} t}{1-d_{2} t-\frac{c_{2} t}{1-\cdots}}},
$$

and of the Jacobi type (or J-fractions)

$$
\sum_{n \geqslant 0} a_{n} t^{n}=\frac{1}{1-e_{0} t-\frac{f_{1} t^{2}}{1-e_{1} t-\frac{f_{2} t^{2}}{1-\cdots}}},
$$

once again interpreted as formal power series in $t$. We will find some recurrences of the GKP type (1.1) whose ogf can be expressed as a T-fraction (or J-fraction) in which the coefficients $\boldsymbol{c}=\left(c_{i}\right)_{i \geqslant 1}$ and $\boldsymbol{d}=\left(d_{i}\right)_{i \geqslant 1}$ (or $\boldsymbol{e}=\left(e_{i}\right)_{i \geqslant 0}$ and $\boldsymbol{f}=\left(f_{i}\right)_{i \geqslant 1}$ ) are polynomials in the indeterminates $x$ and $\boldsymbol{\mu}$. However, this list is almost certainly incomplete; we have not attempted to make a complete determination, for reasons that will be explained later. 
This paper is organized as follows: In Section 2 we analyze the symmetry group of the GKP recurrence. In Section 3 we completely characterize those GKP recurrences whose ogf has an S-fraction representation in which the coefficients are polynomials in $x$. In Section 4 we review some transformation formulae that will be needed for our discussion of T-fractions and J-fractions. In Section 5 we show some examples (but not a complete characterization) of GKP recurrences whose ogf has a T-fraction or J-fraction representation in which the coefficients are polynomials in $x$.

Finally, in Section 6 we propose some problems for future work. In particular, one motivation for the continued-fraction expansions studied here is a conjecture [50] concerning the coefficientwise Hankel-total positivity $[48,49]$ of the row-generating polynomials $P_{n}(x ; \boldsymbol{\mu})$ of the GKP recurrence. Section 6.2 is devoted to introducing and discussing this conjecture.

Appendix A provides some more details concerning the matrix product of two GKP arrays (as well as some more general arrays), while Appendix B gives a general treatment of inverse pairs of lower-triangular arrays. Finally, in the Supplementary Material we provide details of the computer-assisted search that constitutes the first stage of the proof of Theorem 3.1.

\section{Symmetries of the GKP recurrence}

In this section we will treat some symmetries of the GKP recurrence: by this we mean maps $M: \boldsymbol{\mu} \mapsto \boldsymbol{\mu}^{\prime}$ for which the array $\boldsymbol{T}\left(\boldsymbol{\mu}^{\prime}\right)$ can be written in a simple way in terms of $\boldsymbol{T}(\boldsymbol{\mu})$. By "simple" we mean that the row-generating polynomials $P_{n}\left(x ; \boldsymbol{\mu}^{\prime}\right)$ can be written as a Möbius transformation of $P_{n}(x ; \boldsymbol{\mu})$ :

$$
P_{n}\left(x ; \boldsymbol{\mu}^{\prime}\right)=[c(\boldsymbol{\mu}) x+d(\boldsymbol{\mu})]^{n} P_{n}\left(\frac{a(\boldsymbol{\mu}) x+b(\boldsymbol{\mu})}{c(\boldsymbol{\mu}) x+d(\boldsymbol{\mu})}\right)
$$

for some functions $a(\boldsymbol{\mu}), b(\boldsymbol{\mu}), c(\boldsymbol{\mu}), d(\boldsymbol{\mu})$. We do not purport to find all such transformations, but we will find a fairly large (48-element) discrete group of them. Some (but not all) of the transformations we will consider are involutions, i.e. satisfy $M(M(\boldsymbol{\mu}))=\boldsymbol{\mu}$.

\subsection{Some special symmetries}

The most obvious symmetry is:

Scaling. For parameters $\kappa$ and $\lambda$, define the map $S_{\kappa, \lambda}$ by

$$
S_{\kappa, \lambda}\left(\alpha, \beta, \gamma, \alpha^{\prime}, \beta^{\prime}, \gamma^{\prime}\right)=\left(\kappa \alpha, \kappa \beta, \kappa \gamma, \lambda \alpha^{\prime}, \lambda \beta^{\prime}, \lambda \gamma^{\prime}\right) .
$$

Then we have the obvious relation

$$
T\left(n, k ; S_{\kappa, \lambda} \boldsymbol{\mu}\right)=\kappa^{n-k} \lambda^{k} T(n, k ; \boldsymbol{\mu}) .
$$

Here the parameters $\kappa$ and $\lambda$ can be indeterminates, or they can be real or complex numbers. All the maps $S_{\kappa, \lambda}$ trivially commute: we have $S_{\kappa, \lambda} S_{\kappa^{\prime}, \lambda^{\prime}}=S_{\kappa \kappa^{\prime}, \lambda \lambda^{\prime}}$. Thus, if 
we consider $\kappa$ and $\lambda$ to be nonzero complex numbers, then the maps $S_{\kappa, \lambda}$ form a group isomorphic to $\mathbb{C}^{*} \times \mathbb{C}^{*}$, where $\mathbb{C}^{*} \stackrel{\text { def }}{=} \mathbb{C} \backslash\{0\}$.

Later, we shall use in particular the scaling maps $S=S_{-1,1}, S^{\prime}=S_{1,-1}$ and $S S^{\prime}=$ $S_{-1,-1}$. Of course these are commuting involutions, which generate a group $\left\{1, S, S^{\prime}, S S^{\prime}\right\}$ isomorphic to $\mathbb{Z}_{2} \times \mathbb{Z}_{2}$.

Let us now turn to some discrete symmetries of the GKP recurrence. The most important of these is:

Duality $[2,57-59]$. Given a triangular array $\boldsymbol{T}=(T(n, k))_{0 \leqslant k \leqslant n}$, let us define the dual (or reversed) array $\boldsymbol{T}^{*}=\left(T^{*}(n, k)\right)_{0 \leqslant k \leqslant n}$ by

$$
T^{*}(n, k) \stackrel{\text { def }}{=} T(n, n-k) .
$$

The map $\boldsymbol{T} \mapsto \boldsymbol{T}^{*}$ is obviously an involution, i.e. $\left(\boldsymbol{T}^{*}\right)^{*}=\boldsymbol{T}$. In the GKP recurrence, the duality map $\boldsymbol{T} \mapsto \boldsymbol{T}^{*}$ can be implemented by the transformation of parameters [57, p. 67 , Proposition 3.1.1]

$$
\boldsymbol{\mu} \mapsto D \boldsymbol{\mu} \stackrel{\text { def }}{=}\left(\alpha^{\prime}+\beta^{\prime},-\beta^{\prime}, \gamma^{\prime}, \alpha+\beta,-\beta, \gamma\right)
$$

That is, we have

$$
T(n, k ; D \boldsymbol{\mu})=T(n, n-k ; \boldsymbol{\mu}) .
$$

Of course, the map $D$ is also an involution, i.e. $D(D(\boldsymbol{\mu}))=\boldsymbol{\mu}$. The corresponding generating functions transform as

$$
\begin{aligned}
P_{n}(x ; D \boldsymbol{\mu}) & =x^{n} P_{n}\left(\frac{1}{x} ; \boldsymbol{\mu}\right) \\
f(x, t ; D \boldsymbol{\mu}) & =f\left(\frac{1}{x}, x t ; \boldsymbol{\mu}\right) \\
F(x, t ; D \boldsymbol{\mu}) & =F\left(\frac{1}{x}, x t ; \boldsymbol{\mu}\right)
\end{aligned}
$$

Remark. Neuwirth's [40] case $\alpha^{\prime}=0$ is dual to Spivey's [52] case (S1) $\beta=-\alpha$, while Spivey's [52] cases (S2) $\beta=\beta^{\prime}=0$ and (S3) $\alpha / \beta=\alpha^{\prime} / \beta^{\prime}+1$ are self-dual.

Another important involution is the following:

Zhu involution [67]. Define the map $Z: \boldsymbol{\mu} \mapsto Z \boldsymbol{\mu}$ by

$$
Z\left(\alpha, \beta, \gamma, \alpha^{\prime}, \beta^{\prime}, \gamma^{\prime}\right) \stackrel{\text { def }}{=}\left(\alpha-\frac{\beta}{\beta^{\prime}} \alpha^{\prime},-\beta,-\beta+\gamma-\frac{\beta}{\beta^{\prime}} \gamma^{\prime}, \alpha^{\prime}, \beta^{\prime}, \gamma^{\prime}\right)
$$

A simple computation shows that $Z$ is an involution, i.e. $Z(Z(\boldsymbol{\mu}))=\boldsymbol{\mu}$. Here we should either consider $\boldsymbol{\mu}$ to be indeterminates and work in the polynomial ring $\mathbb{Z}\left[\boldsymbol{\mu},\left(\beta^{\prime}\right)^{-1}\right]$, or 
else consider $\boldsymbol{\mu}$ to be real or complex numbers subject to the condition $\beta^{\prime} \neq 0$. The triangular array $\boldsymbol{T}$ transforms as

$$
\begin{aligned}
T(n, k ; Z \boldsymbol{\mu}) & =\sum_{j=k}^{n} T(n, j ; \boldsymbol{\mu})\left(\begin{array}{l}
j \\
k
\end{array}\right)\left(-\frac{\beta}{\beta^{\prime}}\right)^{j-k} \\
T(n, k ; \boldsymbol{\mu}) & =\sum_{j=k}^{n} T(n, j ; Z \boldsymbol{\mu})\left(\begin{array}{l}
j \\
k
\end{array}\right)\left(\frac{\beta}{\beta^{\prime}}\right)^{j-k}
\end{aligned}
$$

This has a simple expression in terms of the row-generating polynomials:

$$
P_{n}(x ; Z \boldsymbol{\mu})=P_{n}\left(x-\frac{\beta}{\beta^{\prime}} ; \boldsymbol{\mu}\right) .
$$

Equivalently, the generating functions transform as

$$
\begin{aligned}
& f(x, t ; Z \boldsymbol{\mu})=f\left(x-\frac{\beta}{\beta^{\prime}}, t ; \boldsymbol{\mu}\right) \\
& F(x, t ; Z \boldsymbol{\mu})=F\left(x-\frac{\beta}{\beta^{\prime}}, t ; \boldsymbol{\mu}\right)
\end{aligned}
$$

The identity (2.9a) is a special case of Corollary A.15 in Appendix A.

Let us observe that that $(2.9 \mathrm{a})$ can be written as a matrix product

$$
\boldsymbol{T}(Z \boldsymbol{\mu})=\boldsymbol{T}(\boldsymbol{\mu}) B_{-\beta / \beta^{\prime}}
$$

where $B_{\xi}$ denotes the $\xi$-binomial matrix

$$
B_{\xi}(n, k)=\left(\begin{array}{l}
n \\
k
\end{array}\right) \xi^{n-k}
$$

Equivalently, $(2.9 \mathrm{~b})$ can be written as $\boldsymbol{T}(\boldsymbol{\mu})=\boldsymbol{T}(Z \boldsymbol{\mu}) B_{\beta / \beta^{\prime}}$. Please note that the $\xi$ binomial matrix satisfies $B_{\xi} B_{\xi^{\prime}}=B_{\xi^{\prime}} B_{\xi}=B_{\xi+\xi^{\prime}}$ and hence in particular $\left(B_{\xi}\right)^{-1}=B_{-\xi}$.

A brute-force computation using $n=0,1,2,3$ shows that the only solutions to the equations $P_{n}\left(x ; \boldsymbol{\mu}^{\prime}\right)=P_{n}(x+\xi ; \boldsymbol{\mu})$ valid for generic parameters $\boldsymbol{\mu}$ are the identity map $\left(\xi=0, \boldsymbol{\mu}^{\prime}=\boldsymbol{\mu}\right)$ and the map $(2.8) /(2.10)$.

The dual of the Zhu involution is:

"Riordan" involution $\boldsymbol{R}=\boldsymbol{D} \boldsymbol{Z} \boldsymbol{D}$ [3, Proposition 17]. We have

$$
R\left(\alpha, \beta, \gamma, \alpha^{\prime}, \beta^{\prime}, \gamma^{\prime}\right) \stackrel{\text { def }}{=}\left(\alpha, \beta, \gamma, \alpha^{\prime}+\beta^{\prime}-\frac{\beta^{\prime}}{\beta} \alpha,-\beta^{\prime}, \gamma^{\prime}+\beta^{\prime}-\frac{\beta^{\prime}}{\beta} \gamma\right) .
$$


Here we should either consider $\boldsymbol{\mu}$ to be indeterminates and work in the polynomial ring $\mathbb{Z}\left[\boldsymbol{\mu}, \beta^{-1}\right]$, or else consider $\boldsymbol{\mu}$ to be real or complex numbers subject to the condition $\beta \neq 0$. The triangular array $\boldsymbol{T}$ transforms as

$$
\begin{aligned}
T(n, k ; R \boldsymbol{\mu}) & =\sum_{j=0}^{k} T(n, j ; \boldsymbol{\mu})\left(\begin{array}{l}
n-j \\
n-k
\end{array}\right)\left(-\frac{\beta^{\prime}}{\beta}\right)^{k-j} \\
T(n, k ; \boldsymbol{\mu}) & =\sum_{j=0}^{k} T(n, j ; R \boldsymbol{\mu})\left(\begin{array}{l}
n-j \\
n-k
\end{array}\right)\left(\frac{\beta^{\prime}}{\beta}\right)^{k-j}
\end{aligned}
$$

This has a simple expression in terms of the row-generating polynomials:

$$
P_{n}(x ; R \boldsymbol{\mu})=\left(\frac{\beta-\beta^{\prime} x}{\beta}\right)^{n} P_{n}\left(\frac{\beta x}{\beta-\beta^{\prime} x} ; \boldsymbol{\mu}\right) .
$$

We say that $\boldsymbol{T}(\boldsymbol{\mu})$ and $\boldsymbol{T}(R \boldsymbol{\mu})$ form an inverse pair of lower-triangular arrays: see Appendix B.

\subsection{Determination of the symmetry group}

Let us now consider the discrete group $\mathcal{G}$ generated by the three involutions $S, D$ and $Z$. (This group also includes $S^{\prime}=D S D$ and $R=D Z D$.) Of course $S^{2}=D^{2}=Z^{2}=$ $\left(S^{\prime}\right)^{2}=1$. It is easy to see that

$$
\begin{aligned}
& S Z=Z S \\
& S^{\prime} Z=Z S^{\prime} \\
& S S^{\prime}=S^{\prime} S \\
& (D S)^{2}=(S D)^{2}=S S^{\prime}
\end{aligned}
$$

and hence $(D S)^{4}=(S D)^{4}=1$. A slightly more involved computation shows that

$$
(D Z)^{6}=(Z D)^{6}=S S^{\prime}
$$

and hence $(D Z)^{12}=(Z D)^{12}=1$. This motivates defining the group element

$$
X \stackrel{\text { def }}{=} D Z,
$$

which acts on $\boldsymbol{\mu}$ as

$$
X\left(\alpha, \beta, \gamma, \alpha^{\prime}, \beta^{\prime}, \gamma^{\prime}\right)=\left(\alpha^{\prime}+\beta^{\prime},-\beta^{\prime}, \gamma^{\prime}, \alpha-\beta-\frac{\beta}{\beta^{\prime}} \alpha^{\prime}, \beta, \gamma-\beta-\frac{\beta}{\beta^{\prime}} \gamma^{\prime}\right)
$$

and on the row-generating polynomials as

$$
P_{n}(x ; X \boldsymbol{\mu})=x^{n} P_{n}\left(\frac{1}{x}-\frac{\beta}{\beta^{\prime}} ; \boldsymbol{\mu}\right) .
$$


We therefore consider $\mathcal{G}$ as generated by $X$ together with the two commuting involutions $S$ and $Z$. These generators satisfy the relations

$$
\begin{aligned}
X^{12} & =1 \\
S^{2} & =1 \\
Z^{2} & =1 \\
S Z & =Z S \\
S X S & =X^{7} \\
Z X Z & =X^{11}=X^{-1}
\end{aligned}
$$

and we shall see shortly that there are no other independent relations. Brute-force computation shows that the group $\mathcal{G}$ has 48 elements, each of which corresponds to a distinct transformation $\boldsymbol{\mu} \mapsto \boldsymbol{\mu}^{\prime}$. There are two central elements: the identity element 1 , and the nontrivial central element $X^{6}=S S^{\prime}$. The conjugacy classes of $\mathcal{G}$ are as follows (here the integer labeling a conjugacy class denotes the order of its elements):

- Class 1 (central, order 1 , size 1$):\{1\}$

- Class 2a (central, order 2, size 1): $\left\{X^{6}\right\}$

- Class 2b (order 2, size 2): $\left\{S, S X^{6}\right\}$

- Class 2c (order 2, size 2): $\left\{S X^{3}, S X^{9}\right\}$

- Class 2d (order 2, size 3): $\left\{S Z, S Z X^{4}, S Z X^{8}\right\}$

- Class 2e (order 2, size 3): $\left\{S Z X^{2}, S Z X^{6}, S Z X^{10}\right\}$

- Class $2 \mathrm{f}$ (order 2, size 6): $\left\{Z, Z X^{2}, Z X^{4}, Z X^{6}, Z X^{8}, Z X^{10}\right\}$

- Class $2 \mathrm{~g}$ (order 2, size 6): $\left\{Z X, Z X^{3}, Z X^{5}, Z X^{7}, Z X^{9}, Z X^{11}\right\}$

- Class 3 (order 3 , size 2): $\left\{X^{4}, X^{8}\right\}$

- Class 4a (order 4 , size 2): $\left\{X^{3}, X^{9}\right\}$

- Class 4b (order 4, size 6): $\left\{S Z X, S Z X^{3}, S Z X^{5}, S Z X^{7}, S Z X^{9}, S Z X^{11}\right\}$

- Class 6a (order 6, size 2): $\left\{X^{2}, X^{10}\right\}$

- Class 6b (order 6, size 4): $\left\{S X, S X^{5}, S X^{7}, S X^{11}\right\}$

- Class 6c (order 6, size 4): $\left\{S X^{2}, S X^{4}, S X^{8}, S X^{10}\right\}$

- Class 12 (order 12, size 4): $\left\{X, X^{5}, X^{7}, X^{11}\right\}$ 
Let us now show that the group $\mathcal{G}$ can be identified as the direct product $S_{3} \times D_{4}$, where $S_{3}$ is the permutation group on three letters (or equivalently, the dihedral group $D_{3}$ of symmetries of an equilateral triangle) and $D_{4}$ is the dihedral group of symmetries of the square:

$$
\begin{aligned}
& S_{3}=\left\langle a, b: a^{3}=b^{2}=1, b a b=a^{-1}\right\rangle \\
& D_{4}=\left\langle c, d: c^{4}=d^{2}=1, d c d=c^{-1}\right\rangle
\end{aligned}
$$

and hence

$$
\begin{gathered}
S_{3} \times D_{4}=\left\langle a, b, c, d: a^{3}=b^{2}=c^{4}=d^{2}=1, b a b=a^{-1}, d c d=c^{-1},\right. \\
a c=c a, a d=d a, b c=c b, b d=d b\rangle .
\end{gathered}
$$

Assuming temporarily that $\mathcal{G} \simeq S_{3} \times D_{4}$, let us find the presentation. Since $a$ is an element of order 3 , we must have $a=X^{4}$ or its inverse $X^{8}$; the two choices are equivalent, so let us choose $a=X^{4}$. Since $c$ is an element of order 4 that commutes with $a$, we must have $c=X^{3}$ or its inverse $X^{9}$ (the other order- 4 elements are ruled out because $S Z$ does not commute with $X^{4}$ : from $(2.22 \mathrm{e}, \mathrm{f})$ we see that $\left.S Z X^{4} S Z=X^{-4}\right)$; the two choices are equivalent, so let us choose $c=X^{3}$. Note that $c^{2}=X^{6}$ is a central element, as it should be, and that $a$ commutes with $c$. Then $b$ has to be a non-central order-2 element that commutes with $X^{3}$, and $d$ has to be a non-central order-2 element that commutes with $X^{4}$. Looking at classes $2 \mathrm{~b}-2 \mathrm{~g}$ and using $(2.22 \mathrm{~d}, \mathrm{e}, \mathrm{f})$, we see that the only choices are $b=S Z X^{2 m}$ for $m \in\{0,1,2,3,4,5\}$, and $d=S X^{3 n}$ for $n \in\{0,1,2,3\}$. It is easily checked that all of these choices satisfy $b d=d b, b a b=a^{-1}$ and $d c d=c^{-1}$. For $b$ there are two inequivalent choices:

- Choosing $m$ even means that the group $S_{3}$ generated by $a$ and $b$ is $\left\{1, X^{4}, X^{8}\right.$, $\left.S Z, S Z X^{4}, S Z X^{8}\right\}$. We might as well choose $m=0$, hence $b=S Z$.

- Choosing $m$ odd means that the group $S_{3}$ generated by $a$ and $b$ is $\left\{1, X^{4}, X^{8}\right.$, $\left.S Z X^{2}, S Z X^{6}, S Z X^{10}\right\}=\left\{1, X^{4}, X^{8}, S^{\prime} Z, S^{\prime} Z X^{4}, S^{\prime} Z X^{8}\right\}$ We might as well choose $m=3$, hence $b=S Z X^{6}=S^{\prime} Z$.

The two inequivalent choices for $b$ are related by duality $S^{\prime}=D S D$, so there is no loss of generality in taking $b=S Z$. On the other hand, all four choices of $d$ give rise to the same group $D_{4}$ generated by $c$ and $d$, namely $\left\{1, X^{3}, X^{6}, X^{9}, S, S X^{3}, S X^{6}, S X^{9}\right\}$. So we might as well choose $n=0$, hence $d=S$. We are therefore led to conjecture that $\mathcal{G} \simeq S_{3} \times D_{4}$ with the identifications

$$
a=X^{4}, \quad b=S Z, \quad c=X^{3}, \quad d=S .
$$

Since we have already checked all the relations (2.24), and we have verified that $\mathcal{G}$ has 48 elements - the same as $S_{3} \times D_{4}$ — the conjecture is proven.

Within the group $\mathcal{G}$, a special role is played by the subgroup $\mathcal{G}_{0}$ generated by $S$ and $D$ (or equivalently by $S, S^{\prime}$ and $D$ ):

$$
\mathcal{G}_{0}=\left\{1, S, S^{\prime}, S S^{\prime}, D, S D, S^{\prime} D, S S^{\prime} D\right\} .
$$


This is a dihedral group $D_{4}$ : in the presentation (2.23b) we can take $c=S D$ and $d=S$. Please note, however, that this subgroup $D_{4}$ is not the "canonical" subgroup $D_{4}$ that arises in the direct product $\mathcal{G} \simeq S_{3} \times D_{4}$ with the identifications (2.25). It is also useful to consider the conjugate subgroups

$$
\begin{aligned}
\mathcal{G}_{k} \stackrel{\text { def }}{=} X^{-k} \mathcal{G}_{0} X^{k} & =\left\{1, S, S^{\prime}, S S^{\prime}, D X^{2 k}, S D X^{2 k}, S^{\prime} D X^{2 k}, S S^{\prime} D X^{2 k}\right\} \\
& =\left\{1, S, S^{\prime}, S S^{\prime}, Z X^{2 k-1}, S Z X^{2 k-1}, S^{\prime} Z X^{2 k-1}, S S^{\prime} Z X^{2 k-1}\right\}
\end{aligned}
$$

for $k \in \mathbb{Z}$. Since $\mathcal{G}_{k}$ involves $X^{2 k}$, and since the central element $X^{6}=S S^{\prime}$ belongs to $\mathcal{G}_{0}$, the subgroup $\mathcal{G}_{k}$ depends only on $k \bmod 3$, so it suffices to consider $k=0,1,2$. Note that all three subgroups $\mathcal{G}_{k}$ contain the abelian subgroup $\left\{1, S, S^{\prime}, S S^{\prime}\right\}$ and in particular the central element $S S^{\prime}=X^{6}$.

The special role played by the subgroup $\mathcal{G}_{0}$ is the following: If we examine the formulae $M \boldsymbol{\mu}$ for all elements $M \in \mathcal{G}$, we find that $M \boldsymbol{\mu}$ is a polynomial in $\boldsymbol{\mu}$ if and only if $M \in \mathcal{G}_{0}$. In the other cases:

- $M \boldsymbol{\mu}$ is a polynomial in $\boldsymbol{\mu}$ and $\left(\beta^{\prime}\right)^{-1}$ when $M \in\left\{X, X^{7}, S X, S X^{7}, Z, Z X^{6}, S Z\right.$, $\left.S Z X^{6}\right\}$. This is the right coset $\mathcal{G}_{0} X$, or equivalently the left coset $X \mathcal{G}_{1}$.

- $M \boldsymbol{\mu}$ is a polynomial in $\boldsymbol{\mu}$ and $\beta^{-1}$ when $M \in\left\{X^{5}, X^{11}, S X^{5}, S X^{11}, Z X^{4}, Z X^{10}\right.$, $\left.S Z X^{4}, S Z X^{10}\right\}=\left\{X, X^{7}, S X, S X^{7}, R, R X^{6}, S R, S R X^{6}\right\}$. This is the right coset $\mathcal{G}_{0} X^{5}$, or equivalently the left $\operatorname{coset} X^{5} \mathcal{G}_{5}=X^{5} \mathcal{G}_{2}$.

- $M \boldsymbol{\mu}$ is a polynomial in $\boldsymbol{\mu}, \beta^{-1}$ and $\left(\beta^{\prime}\right)^{-1}$ when $M$ is any of the other 24 elements of $\mathcal{G}$. These are the right cosets $\mathcal{G}_{0} X^{k}$, or equivalently the left cosets $X^{k} \mathcal{G}_{k}$, for $k=2,3,4$.

Remark. The group $S_{3} \times D_{4}$ has many other aliases: see e.g. [14].

\subsection{Further scaling properties of the GKP recurrence}

In Section 2.1 we studied several symmetries of the GKP recurrence, and in particular the behavior of the triangular-array entries $T(n, k)$ under the trivial scaling map $S_{\kappa, \lambda}$ defined in $(2.2) /(2.3)$. We would now like to point out some less obvious scaling properties. These properties are most naturally formulated in the first instance for the more general "binomial-like" recurrence

$$
T(n, k)=a_{n, k} T(n-1, k)+a_{n, k}^{\prime} T(n-1, k-1)
$$

for $n \geqslant 1$, again with initial condition $T(0, k)=\delta_{k 0} \cdot{ }^{1}$ Spivey [52, Theorem 1] showed the following:

\footnotetext{
${ }^{1}$ A detailed study of this type of recurrence can be found in the thesis of Théorêt [57]. He uses the term "hyperbinomial" to denote the matrix $\boldsymbol{T}=(T(n, k))_{n, k \geqslant 0}$ satisfying such a recurrence.
} 
Lemma 2.1 (Rescaling of a binomial-like recurrence). Let $\left(a_{n, k}\right),\left(a_{n, k}^{\prime}\right),\left(c_{n}\right),\left(d_{k}\right)$ and $\left(e_{n}\right)$ be indeterminates, and define triangular arrays $(T(n, k))_{0 \leqslant k \leqslant n}$ and $\left(T^{\prime}(n, k)\right)_{0 \leqslant k \leqslant n}$ by the recurrences

$$
\begin{aligned}
T(n, k) & =a_{n, k} T(n-1, k)+a_{n, k}^{\prime} T(n-1, k-1) \\
T^{\prime}(n, k) & =c_{n-k} e_{n} a_{n, k} T^{\prime}(n-1, k)+d_{k} e_{n} a_{n, k}^{\prime} T^{\prime}(n-1, k-1)
\end{aligned}
$$

for $n \geqslant 1$, with initial conditions $T(0, k)=T^{\prime}(0, k)=\delta_{k 0}$. Then

$$
T^{\prime}(n, k)=c_{1} \cdots c_{n-k} d_{1} \cdots d_{k} e_{1} \cdots e_{n} T(n, k) .
$$

Sketch of proof. Represent $T(N, K)$ as a sum over walks in $\mathbb{N} \times \mathbb{N}$ from $(0,0)$ to $(N, K)$, where a "level step" $(n-1, k) \rightarrow(n, k)$ gets a weight $a_{n, k}$ and a "rise" $(n-1, k-1) \rightarrow$ $(n, k)$ gets a weight $a_{n, k}^{\prime}$, and the weight of a walk is the product of the weights of its steps; and analogously for $T^{\prime}(N, K)$. The result (2.31) then follows by simple arguments [49]. Alternatively $[52,53]$ one can show that the quantities (2.31) satisfy the recurrence (2.30).

Let us now specialize Lemma 2.1 to the GKP recurrence

$$
a_{n, k}=\alpha n+\beta k+\gamma, \quad a_{n, k}^{\prime}=\alpha^{\prime} n+\beta^{\prime} k+\gamma^{\prime} .
$$

It is easy to see that there are precisely three cases in which the recurrence (2.30) for $T^{\prime}(n, k)$ is also of the GKP form:

(a) $\alpha=\beta=0$ and $c_{n}=\kappa n+\lambda, d_{k}=1, e_{n}=1$

(b) $\alpha^{\prime}=\beta^{\prime}=0$ and $d_{k}=\kappa k+\lambda, c_{n}=e_{n}=1$

(c) $\alpha=\beta=\alpha^{\prime}=\beta^{\prime}=0$ and $e_{n}=\kappa n+\lambda, c_{n}=1, d_{k}=1$

We thus obtain:

Corollary 2.2 (Rescaling of a GKP recurrence). Let $\alpha, \beta, \gamma, \alpha^{\prime}, \beta^{\prime}, \gamma^{\prime}$ and $\kappa, \lambda$ be indeterminates. Then:

(a) When $\alpha=\beta=0$ we have

$$
\begin{aligned}
& T\left(n, k ; \kappa \gamma, 0, \lambda \gamma, \alpha^{\prime}, \beta^{\prime}, \gamma^{\prime}\right)= \\
& (\kappa+\lambda)(2 \kappa+\lambda) \cdots[(n-k) \kappa+\lambda] T\left(n, k ; 0,0, \gamma, \alpha^{\prime}, \beta^{\prime}, \gamma^{\prime}\right) .
\end{aligned}
$$

(b) When $\alpha^{\prime}=\beta^{\prime}=0$ we have

$$
\begin{aligned}
& T\left(n, k ; \alpha, \beta, \gamma, 0, \kappa \gamma^{\prime}, \lambda \gamma^{\prime}\right)= \\
& (\kappa+\lambda)(2 \kappa+\lambda) \cdots(k \kappa+\lambda) T\left(n, k ; \alpha, \beta, \gamma, 0,0, \gamma^{\prime}\right) .
\end{aligned}
$$

(c) When $\alpha=\beta=\alpha^{\prime}=\beta^{\prime}=0$ we have

$$
\begin{aligned}
& T\left(n, k ; \kappa \gamma, 0, \lambda \gamma, \kappa \gamma^{\prime}, 0, \lambda \gamma^{\prime}\right)= \\
& (\kappa+\lambda)(2 \kappa+\lambda) \cdots(n \kappa+\lambda) T\left(n, k ; 0,0, \gamma, 0,0, \gamma^{\prime}\right) .
\end{aligned}
$$




\section{Characterization of the GKP recurrences with an S-fraction representation}

Our main goal in this paper is to determine all families of parameters $\boldsymbol{\mu} \in \mathbb{C}^{6}$ such that the corresponding ogf (1.3) has an S-type continued fraction (1.7) with coefficients $c_{1}, c_{2}, \ldots$ that are polynomials in $x$ (rather than rational functions). Our findings can be summarized in the following:

Theorem 3.1. Let $\boldsymbol{\mu}=\left(\alpha, \beta, \gamma, \alpha^{\prime}, \beta^{\prime}, \gamma^{\prime}\right) \in \mathbb{C}^{6}$, and let $\boldsymbol{T}(\boldsymbol{\mu})=(T(n, k ; \boldsymbol{\mu}))_{0 \leqslant k \leqslant n}$ be the triangular array determined via the recurrence (1.1). Then the corresponding ogf $f(x, t ; \boldsymbol{\mu})$ has a nonterminating $S$-type continued fraction representation (in the sense of formal power series in the indeterminate $t$ )

$$
f(x, t ; \boldsymbol{\mu})=\sum_{n=0}^{\infty} P_{n}(x ; \boldsymbol{\mu}) t^{n}=\frac{1}{1-\frac{c_{1} t}{1-\frac{c_{2} t}{1-\cdots}}}
$$

with coefficients $c_{1}, c_{2}, \ldots$ that are polynomials in $x$ only if the parameter $\boldsymbol{\mu}$ belongs to one or more of the following families:

F1a. $\boldsymbol{\mu}=\left(0, \beta, 0, \alpha^{\prime},-\alpha^{\prime}, \gamma^{\prime}\right)$

F1b. $\boldsymbol{\mu}=\left(0, \beta, \gamma, \alpha^{\prime},-\alpha^{\prime}, 0\right)$

F2a. $\boldsymbol{\mu}=\left(\alpha,-\alpha,-\alpha, \alpha^{\prime}, \beta^{\prime}, \gamma^{\prime}\right)$

F2b. $\boldsymbol{\mu}=\left(\alpha, \beta, \gamma, 0, \beta^{\prime},-\beta^{\prime}\right)$

F3a. $\boldsymbol{\mu}=\left(0, \beta, 0,0, \beta^{\prime}, \gamma^{\prime}\right)$

F3b. $\boldsymbol{\mu}=\left(\alpha,-\alpha, \gamma, \alpha^{\prime},-\alpha^{\prime}, 0\right)$

F4a. $\boldsymbol{\mu}=\left(0, \kappa \beta^{\prime}, \kappa\left(\beta^{\prime}+\gamma^{\prime}\right), 0, \beta^{\prime}, \gamma^{\prime}\right)$

F4b. $\boldsymbol{\mu}=(\alpha,-\alpha, \gamma, \kappa \alpha,-\kappa \alpha, \kappa(\alpha+\gamma))$

F5. $\boldsymbol{\mu}=\left(\alpha, 0, \gamma, \alpha^{\prime}, 0, \gamma^{\prime}\right)$

F6. $\boldsymbol{\mu}=\left(\kappa\left(\alpha^{\prime}+\beta^{\prime}\right), \kappa \beta^{\prime}, \kappa \gamma^{\prime}, \alpha^{\prime}, \beta^{\prime}, \gamma^{\prime}\right)$

Moreover, in all these cases $f(x, t ; \boldsymbol{\mu})$ does have a representation (3.1) with coefficients $c_{1}, c_{2}, \ldots$ that are polynomials in $x$ (though in some degenerate cases this continued fraction might be terminating).

Here families 1a and $1 \mathrm{~b}$ are duals of each other, and likewise for the pairs (2a,2b), $(3 \mathrm{a}, 3 \mathrm{~b})$ and $(4 \mathrm{a}, 4 \mathrm{~b})$; families 5 and 6 are self-dual. Note that families 1a, 1b, 3a, 3b, $4 \mathrm{a}, 4 \mathrm{~b}$ have three free parameters, while families $2 \mathrm{a}, 2 \mathrm{~b}, 5,6$ have four free parameters; that is, the submanifolds in $\boldsymbol{\mu}$-space are of (complex) dimension 3 and 4 , respectively. However, we shall see later that in families $2 \mathrm{a}, 2 \mathrm{~b}$ and 6 some of the parameters are redundant in the sense that they have no effect on the matrix $\boldsymbol{T}(\boldsymbol{\mu})$ [or therefore on the 
coefficients $c_{i}$. In these cases the manifold of matrices $\boldsymbol{T}(\boldsymbol{\mu})$ is of lower dimension than the manifold of parameters $\boldsymbol{\mu}$ : it is of (complex) dimension 2 for families $2 \mathrm{a}$ and $2 \mathrm{~b}$, and (complex) dimension 3 for family 6 . Note, finally, that these families have some overlaps: for instance, family 1 a at $\alpha^{\prime}=0$ coincides with family 3 a at $\beta^{\prime}=0$; and family 2 a at $\alpha=\alpha^{\prime}=0$ coincides with family 3 a at $\beta=0$.

Having already observed how duality $(D)$ acts on our ten families, we can also work out how the rest of the group $\mathcal{G}$ acts on them. Obviously $S$ and $S^{\prime}$ map each family into itself. The Zhu involution $Z$ maps $1 \mathrm{a} \leftrightarrow 4 \mathrm{~b}, 1 \mathrm{~b} \leftrightarrow 3 \mathrm{~b}, 2 \mathrm{a} \leftrightarrow 6$, $3 \mathrm{a} \leftrightarrow 4 \mathrm{a}$, and $2 \mathrm{~b}$ into itself; $Z$ cannot be defined on family 5 because it requires $\beta^{\prime} \neq 0$. Equivalently, the Riordan involution $R=D Z D$ maps $1 \mathrm{a} \leftrightarrow 3 \mathrm{a}, 1 \mathrm{~b} \leftrightarrow 4 \mathrm{a}, 2 \mathrm{~b} \leftrightarrow 6,3 \mathrm{~b} \leftrightarrow 4 \mathrm{~b}$, and $2 \mathrm{a}$ into itself; $R$ cannot be defined on family 5 because it requires $\beta \neq 0$. Finally, the map $X$ (which is not an involution) maps cyclically $1 \mathrm{a} \rightarrow 4 \mathrm{a} \rightarrow 3 \mathrm{~b} \rightarrow 1 \mathrm{a}, 1 \mathrm{~b} \rightarrow 3 \mathrm{a} \rightarrow 4 \mathrm{~b} \rightarrow 1 \mathrm{~b}$, $2 \mathrm{a} \rightarrow 6 \rightarrow 2 \mathrm{~b} \rightarrow 2 \mathrm{a} ; X$ cannot be defined on family 5 because it requires $\beta^{\prime} \neq 0$. It follows from this that the group $\mathcal{G}$ has one orbit $\{1 \mathrm{a}, 1 \mathrm{~b}, 3 \mathrm{a}, 3 \mathrm{~b}, 4 \mathrm{a}, 4 \mathrm{~b}\}$ and another orbit $\{2 \mathrm{a}, 2 \mathrm{~b}, 6\}$. On family 5 only the subgroup $\mathcal{G}_{0} \subset \mathcal{G}$ generated by $S, S^{\prime}, D$ acts, and it leaves family 5 invariant.

The details of the continued fractions for each of the above families will be presented in Propositions 3.6-3.11 below. We will find that the coefficients $\boldsymbol{c}=\left(c_{i}\right)_{i \geqslant 1}$ in each Sfraction are not only polynomials in $x$ (with coefficients that might be rational functions of the parameters $\boldsymbol{\mu}$ ); in fact, they are polynomials (with nonnegative integer coefficients) simultaneously in all the relevant variables (in each case some subset of $\alpha, \beta, \gamma, \alpha^{\prime}, \beta^{\prime}, \gamma^{\prime}$ and $\kappa$ as well as $x$ ) when those variables are treated as indeterminates. The restriction to nonterminating S-fractions will be explained in Section 3.1.

The strategy for our proof is the following:

1. Make a computer-assisted search to find all viable candidates to have such an Sfraction representation (see Section 3.1). See the Supplementary Material for a full account of this procedure.

2. Prove (by specializing a continued fraction to be explained in Section 3.2) that the ogf of each candidate found in step 1 indeed has the conjectured S-fraction representation (see Section 3.3).

\subsection{Computer-assisted search}

Let $\boldsymbol{a}=\left(a_{n}\right)_{n \geqslant 0}$ be a sequence in which $a_{0}=1$ and $a_{1}, a_{2}, \ldots$ are indeterminates. Then the ogf $\sum_{n \geqslant 0} a_{n} t^{n}$ can be expressed as an S-fraction (1.7) with coefficients $c_{1}, c_{2}, \ldots$ that are rational functions of $a_{1}, a_{2}, \ldots$ : that is, $c_{n} \in \mathbb{Q}\left(a_{1}, \ldots, a_{n}\right)$. The first few $c_{i}$ are given in (1.8).

If we then substitute $a_{n}=P_{n}(x)$ where the $P_{n}$ are polynomials with complex coefficients in $x$ (and $P_{0}=1$ ), then the coefficients $c_{i}$ are generically rational functions of $x$. We say "generically" because it is possible that some coefficient $c_{i}$ might vanish, in which case the coefficients $c_{j}$ for $j>i$ are ill-defined because their denominator vanishes. 
Here we restrict attention to nonterminating S-fractions, i.e. we insist that no $c_{i}$ vanishes identically. Under this assumption, all the coefficients $c_{i}$ are well-defined and are rational functions of $x$ (with complex coefficients): that is, $c_{i} \in \mathbb{C}(x)$.

We now specialize to the case in which the polynomials $P_{n}$ are those coming from the GKP recurrence $(1.1)$, i.e. $a_{n}=P_{n}(x ; \boldsymbol{\mu})$ for some $\boldsymbol{\mu} \in \mathbb{C}^{6}$. Our goal is to determine the submanifolds in $\mathbb{C}^{6}$ where the coefficients $c_{i}$ are polynomials - and not just rational functions - in $x$. We do this by making a computer-assisted search using MATHEMATICA to find a finite set of viable candidates for such an S-fraction; then we prove, case by case, that all of these candidates work. It is convenient to explain this computer-assisted search by showing explicitly its first few steps.

The row-generating polynomial $P_{n}(x ; \boldsymbol{\mu})$ is a polynomial of degree $n$ in $x$ with coefficients that are (fairly complicated) polynomials with integer coefficients in the parameters $\boldsymbol{\mu}$. For instance, the first few $P_{n}$ are

$$
\begin{aligned}
P_{0}(x)= & 1 \\
P_{1}(x)= & (\alpha+\gamma)+\left(\alpha^{\prime}+\beta^{\prime}+\gamma^{\prime}\right) x \\
P_{2}(x)= & (\alpha+\gamma)(2 \alpha+\gamma) \\
& +\left(4 \alpha \alpha^{\prime}+\beta \alpha^{\prime}+3 \alpha \beta^{\prime}+\beta \beta^{\prime}+3 \gamma \alpha^{\prime}+2 \gamma \beta^{\prime}+3 \alpha \gamma^{\prime}+\beta \gamma^{\prime}+2 \gamma \gamma^{\prime}\right) x \\
& +\left[\left(\alpha^{\prime}+\beta^{\prime}+\gamma^{\prime}\right)\left(2 \alpha^{\prime}+2 \beta^{\prime}+\gamma^{\prime}\right)\right] x^{2}
\end{aligned}
$$

We now insert these $a_{n}=P_{n}(x ; \boldsymbol{\mu})$ into the equations $(1.8)$ giving the coefficients $c_{i}$.

Coefficient $\boldsymbol{c}_{\mathbf{1}}$. The first coefficient $c_{1}=P_{1}(x)$ is of course a polynomial in $x$, which is of degree at most 1. More precisely, it is of degree 1 if $\alpha^{\prime}+\beta^{\prime}+\gamma^{\prime} \neq 0$, of degree 0 if $\alpha^{\prime}+\beta^{\prime}+\gamma^{\prime}=0$ and $\alpha+\gamma \neq 0$, and identically zero if $\alpha^{\prime}+\beta^{\prime}+\gamma^{\prime}=\alpha+\gamma=0$. This third case is now discarded because of our restriction to nonterminating S-fractions; and as will be seen shortly, we will need to consider the first two cases separately.

Coefficient $\boldsymbol{c}_{\mathbf{2}}$. The next coefficient $c_{2}$ is

$$
c_{2}(x)=\frac{P_{2}(x)-P_{1}(x)^{2}}{P_{1}(x)} \stackrel{\text { def }}{=} \frac{Q(x)}{R(x)},
$$

where the denominator $R(x)$ is a polynomial of degree $\leqslant 1$ in $x$ and the numerator $Q(x)$ is a polynomial of degree $\leqslant 2$ in $x$, and the coefficients in these polynomials are polynomial expressions in $\boldsymbol{\mu}$. We need to determine the conditions under which such a rational function $Q(x) / R(x)$ is in fact a polynomial in $x$. Let us explain the idea in general.

Let $Q(x)=\sum_{k=0}^{m} a_{k}(\boldsymbol{\mu}) x^{k}$ and $R(x)=\sum_{k=0}^{n} b_{k}(\boldsymbol{\mu}) x^{k}$ be polynomials in $x$ in which the coefficients $a_{k}$ and $b_{k}$ are polynomial expressions in some set of indeterminates $\boldsymbol{\mu}$. The polynomial remainder $\operatorname{rem}(Q(x), R(x))$ is then well-defined whenever the leading coefficient $b_{n}$ of the denominator polynomial is nonzero, and in this case it is a polynomial in $x$ of degree $<n$. On the other hand, if $b_{n}=0$ and $b_{n-1} \neq 0$, then we must consider 
$R$ as a polynomial of degree $n-1$, yielding a remainder that is of degree $<n-1$; or if $b_{n}=b_{n-1}=0$ and $b_{n-2} \neq 0$, then we must consider $R$ as a polynomial of degree $n-2$, yielding a remainder that is of degree $<n-2$; and so on. The last nontrivial case is $b_{n}=\ldots=b_{1}=0$ and $b_{0} \neq 0$ : then $R$ is a nonzero constant function and $Q / R$ is automatically a polynomial. The completely degenerate case $b_{n}=\ldots=b_{0}=0$ (i.e. $R=0$ ) is excluded by our restriction to nonterminating $\mathrm{S}$-fractions. There are therefore $n+1$ nontrivial cases, corresponding to the order of the highest nonvanishing coefficient $b_{i}(0 \leqslant i \leqslant n)$. For each of these cases, we obtain a set of polynomial equations and inequations in the indeterminates $\boldsymbol{\mu}$ : namely, the equations $b_{n}=\ldots=b_{i+1}=0$, the inequation $b_{i} \neq 0$, and then the equations asserting that all the coefficients of the remainder polynomial are zero (since this remainder polynomial has degree at most $i-1$, there are $i$ such equations). ${ }^{2}$ This system of equations and inequations must then be solved, yielding some algebraic variety in the space of parameters $\boldsymbol{\mu}$ (in our case $\mathbb{C}^{6}$ ).

Let us examine from this point of view the coefficient $c_{2}$ given by (3.3). The numerator and denominator polynomials are

$$
\begin{aligned}
Q(x)=P_{2}(x)-P_{1}(x)^{2}= & \alpha(\alpha+\gamma) \\
& +\left(2 \alpha \alpha^{\prime}+\alpha^{\prime} \beta+\alpha \beta^{\prime}+\beta \beta^{\prime}+\alpha^{\prime} \gamma+\alpha \gamma^{\prime}+\beta \gamma^{\prime}\right) x \\
& +\left(\alpha^{\prime}+\beta^{\prime}\right)\left(\alpha^{\prime}+\beta^{\prime}+\gamma^{\prime}\right) x^{2} \\
R(x)=P_{1}(x)= & (\alpha+\gamma)+\left(\alpha^{\prime}+\beta^{\prime}+\gamma^{\prime}\right) x
\end{aligned}
$$

Note that the polynomial $R(x)$ cannot be identically zero, because $R(x)=c_{1}(x)$ and we have already assumed that $c_{1}(x)$ is not identically zero: that is, $\alpha+\gamma \neq 0$ or $\alpha^{\prime}+\beta^{\prime}+\gamma^{\prime} \neq 0$ or both. So we have either $\operatorname{deg} R=1$ or $\operatorname{deg} R=0$. Then $c_{2}(x)=Q(x) / R(x)$ is a polynomial in $x$ if and only if either

(a) $\operatorname{deg} \boldsymbol{R}=1$ : The leading coefficient $\alpha^{\prime}+\beta^{\prime}+\gamma^{\prime}$ of the denominator polynomial $R(x)$ is nonzero, and the remainder

$$
\operatorname{rem}(Q(x), R(x))=\frac{\alpha+\gamma}{\alpha^{\prime}+\beta^{\prime}+\gamma^{\prime}}\left[(\alpha+\gamma) \beta^{\prime}-\left(\alpha^{\prime}+\beta^{\prime}+\gamma^{\prime}\right) \beta\right]
$$

is zero; or else

(b) $\operatorname{deg} \boldsymbol{R}=0$ : The leading coefficient $\alpha^{\prime}+\beta^{\prime}+\gamma^{\prime}$ of the denominator polynomial $R(x)$ is zero, and the constant term $\alpha+\gamma$ is nonzero. In this case no constraint is imposed on the numerator polynomial $Q(x)$.

Therefore, we should consider three distinct cases:

$\left(\mathrm{a}_{1}\right) \alpha^{\prime}+\beta^{\prime}+\gamma^{\prime} \neq 0$ and $\alpha+\gamma=0$ : Then $\boldsymbol{\mu}=\left(\alpha, \beta,-\alpha, \alpha^{\prime}, \beta^{\prime}, \gamma^{\prime}\right)$ with $\alpha^{\prime}+\beta^{\prime}+\gamma^{\prime} \neq 0$, and $c_{2}(x)=(\alpha+\beta)+\left(\alpha^{\prime}+\beta^{\prime}\right) x$.

\footnotetext{
${ }^{2}$ To compute this remainder polynomial, we work in the $\operatorname{ring} \mathbb{C}(\boldsymbol{\mu})[x]$ of polynomials in $x$ whose coefficients are rational functions (with complex coefficients) in the indeterminates $\boldsymbol{\mu}$.
} 
$\left(\mathrm{a}_{2}\right) \alpha^{\prime}+\beta^{\prime}+\gamma^{\prime} \neq 0$ and $(\alpha+\gamma) \beta^{\prime}=\left(\alpha^{\prime}+\beta^{\prime}+\gamma^{\prime}\right) \beta$ : Then $\boldsymbol{\mu}=\left(\alpha, \frac{(\alpha+\gamma) \beta^{\prime}}{\alpha^{\prime}+\beta^{\prime}+\gamma^{\prime}}, \gamma, \alpha^{\prime}, \beta^{\prime}, \gamma^{\prime}\right)$ with $\alpha^{\prime}+\beta^{\prime}+\gamma^{\prime} \neq 0$, and $c_{2}(x)=\alpha+\left(\alpha^{\prime}+\beta^{\prime}\right) x$.

(b) $\alpha^{\prime}+\beta^{\prime}+\gamma^{\prime}=0$ and $\alpha+\gamma \neq 0$. Then $\boldsymbol{\mu}=\left(\alpha, \beta, \gamma, \alpha^{\prime}, \beta^{\prime},-\left(\alpha^{\prime}+\beta^{\prime}\right)\right)$, and $c_{2}(x)=$ $\alpha+\alpha^{\prime} x$.

In each family, the number of independent parameters in $\boldsymbol{\mu}$ is five. And in each case we will impose, going forward, a disjunction of inequations to guarantee that the polynomial $c_{2}(x)$ is not identically zero: for instance, in case $\left(\mathrm{a}_{1}\right)$ we will impose $\alpha+\beta \neq 0$ or $\alpha^{\prime}+\beta^{\prime} \neq 0$.

Coefficient $\boldsymbol{c}_{\mathbf{3}}$. For each of the three cases encountered in the analysis of $c_{2}$, we consider separately the formula (1.8c) for $c_{3}$, which becomes

$$
c_{3}(x)=\frac{P_{1}(x) P_{3}(x)-P_{2}(x)^{2}}{P_{1}(x)\left[P_{1}(x)^{2}-P_{2}(x)\right]} \stackrel{\text { def }}{=} \frac{Q_{3}(x)}{R_{3}(x)}
$$

for some polynomials $Q_{3}(x)$ and $R_{3}(x)$. If $P_{1}, P_{2}, P_{3}$ were arbitrary polynomials of degree $1,2,3$, respectively, one would expect generically that the numerator polynomial $Q_{3}$ has degree 4 and the denominator polynomial $R_{3}$ has degree 3 . And indeed this is what happens if one inserts the general expressions for $P_{n}(x ; \boldsymbol{\mu})$. However, in the three special cases $\left(\mathrm{a}_{1}\right),\left(\mathrm{a}_{2}\right)$ and $(\mathrm{b})$, the equality conditions cause the degrees of $Q_{3}$ and $R_{3}$ to be reduced to $\leqslant 2$ and $\leqslant 1$, respectively. Furthermore, in all cases the denominator $R_{3}(x)$ turns out to be a nonzero multiple of $c_{2}(x)$; so as a consequence of the inequations imposed at the preceding stage, the polynomial $R_{3}(x)$ cannot be the zero polynomial. Therefore, in each of these three cases the remainder $\operatorname{rem}\left(Q_{3}(x), R_{3}(x)\right)$ is simply a constant, just as it was for $c_{2}$. One thus repeats, for each of these three cases, an analysis along the same lines as was done for $c_{2}$ : firstly distinguishing whether the leading coefficient of $R_{3}$ is nonzero or zero (i.e. whether $\operatorname{deg} R_{3}=1$ or 0 ), and then dividing the first case according to the different factors in the remainder. In each sub-case it turns out that we must impose one additional equality beyond those imposed at the preceding stage; the equation to be solved is always of degree 1 in at least one of the variables, so we can solve for this variable as a (possibly rational) function of the others. At the end one finds that case $\left(a_{1}\right)$ divides into four sub-cases, case $\left(a_{2}\right)$ divides into four sub-cases, and case (b) divides into three sub-cases. In this way we find 11 subfamilies in which $c_{i}(x)$ is a polynomial in $x$ for all $i \leqslant 3$. In ten of the 11 subfamilies there are now four independent parameters in $\boldsymbol{\mu}$, while in one there are only three.

Coefficients $\boldsymbol{c}_{4}, \boldsymbol{c}_{5}, \ldots$. The procedure should by now be clear: at each stage we divide each previously found case into sub-cases, thus forming a decision tree. Some branches of this tree terminate, i.e. the solution set of the system of equations is empty. It was necessary to go up to level $c_{7}$ in order to find all the terminating branches. For each of the branches that survived to level $c_{7}$, we continued this procedure through level $c_{10}$, just to convince ourselves that the candidate was indeed viable. Thus, we declared a 
branch to be viable if, for the corresponding family $\boldsymbol{\mu}$, the coefficients $c_{i}$ for $1 \leqslant i \leqslant 10$ are polynomials in $x$ (always of degree $\leqslant 1$ ) that are not identically vanishing. For each such branch, the coefficients $c_{i}$ turned out to have very simple forms (namely, affine in $i$ separately odd and even $i$ ), from which we were able to conjecture a precise formula for all the coefficients $c_{i}(x ; \boldsymbol{\mu})$.

We also streamlined this procedure as follows: whenever we obtained a branch of the decision tree that is either identical to or a subset of a previously found branch, then we dropped that branch as redundant.

Along the way, we sometimes obtained branches in which the most recent coefficient $c_{i}(x)$ is identically vanishing. These branches correspond to terminating S-fractions and were therefore dropped. Some of these terminating S-fractions are simply special cases of the families $1 \mathrm{a}-6$ of Theorem 3.1, in which one of the coefficients $c_{i}(x)$ happens to vanish. (Indeed, there are infinitely many such special cases, corresponding to the choice of the index $i$ of the vanishing coefficient.) On the other hand, a few of the terminating S-fractions are non-trivial in the sense that they are not simply specializations of the families 1a-6. Since these may be of some interest in their own right, we compile them in Section 4 of the Supplementary Material.

It is also worthy of note that, at every stage in this procedure, the simplification noted above for $c_{3}(x)$ [cf. (3.6)] occurred: namely, the polynomials $Q_{i}(x)$ and $R_{i}(x)$, which one would expect generically to be of degrees $\left(\begin{array}{c}i \\ 2\end{array}\right)+1$ and $\left(\begin{array}{l}i \\ 2\end{array}\right)$, respectively, turned out to have degrees 2 and 1 (or in a few cases even less), as a result of the equality conditions imposed at earlier stages. We leave it as an open problem to find a general explanation of this remarkable simplification.

The details of this computer-assisted search are given in the Supplementary Material. Let us stress that our search was "computer-assisted" only in the sense that it used MATHEMATICA to perform elementary algebraic operations such as manipulation of polynomials and rational functions, series expansions, and PolynomialRemainder. We did not need to use any more advanced algebraic functions such as Solve or Reduce. If one assumes the correctness of MATHEMATICA's algebraic manipulations, the rest of the proof is easily human-verifiable (though tedious) and is explained in detail in the Supplementary Material.

The final list of candidates contains 10 members. Seven of them are families 1a, 1b, 2a, $2 \mathrm{~b}, 3 \mathrm{a}, 3 \mathrm{~b}$ and 5 of Theorem 3.1. In these cases, the entries of $\boldsymbol{\mu}$ are linear combinations of a subset of the parameters $\left(\alpha, \beta, \gamma, \alpha^{\prime}, \beta^{\prime}, \gamma^{\prime}\right)$, and the conjectured coefficients $c_{i}(x ; \boldsymbol{\mu})$ are polynomials (with nonnegative integer coefficients) jointly in $x$ and the parameters. Their expressions are:

F1a. $c_{2 k-1}=\left[\gamma^{\prime}+(k-1) \alpha^{\prime}\right] x$ and $c_{2 k}=k \beta$.

F1b. $c_{2 k-1}=\gamma+(k-1) \beta$ and $c_{2 k}=k \alpha^{\prime} x$.

F2a. $c_{2 k-1}=\left[\gamma^{\prime}+k\left(\alpha^{\prime}+\beta^{\prime}\right)\right] x$ and $c_{2 k}=k\left(\alpha^{\prime}+\beta^{\prime}\right) x$.

F2b. $c_{2 k-1}=k \alpha+\gamma$ and $c_{2 k}=k \alpha$.

F3a. $c_{2 k-1}=\left(\gamma^{\prime}+k \beta^{\prime}\right) x$ and $c_{2 k}=k\left(\beta+\beta^{\prime} x\right)$. 
F3b. $c_{2 k-1}=k \alpha+\gamma$ and $c_{2 k}=k\left(\alpha+\alpha^{\prime} x\right)$.

F5. $c_{2 k-1}=\left(\gamma+\gamma^{\prime} x\right)+k\left(\alpha+\alpha^{\prime} x\right)$ and $c_{2 k}=k\left(\alpha+\alpha^{\prime} x\right)$.

For these families we therefore find a posteriori that the S-fraction (3.1) might also be valid when the $\boldsymbol{\mu}$ (or rather, some subset of them) are treated as indeterminates.

The other three cases require additional discussion. For instance, family 6 comes originally from

$$
\boldsymbol{\mu}=\left(\alpha, \frac{\alpha \beta^{\prime}}{\alpha^{\prime}+\beta^{\prime}}, \frac{\alpha \gamma^{\prime}}{\alpha^{\prime}+\beta^{\prime}}, \alpha^{\prime}, \beta^{\prime}, \gamma^{\prime}\right)
$$

leading to the coefficients

$$
c_{2 k-1}=\left[\gamma^{\prime}+k\left(\alpha^{\prime}+\beta^{\prime}\right)\right]\left(\frac{\alpha}{\alpha^{\prime}+\beta^{\prime}}+x\right), \quad c_{2 k}=k\left(\alpha+\left(\alpha^{\prime}+\beta^{\prime}\right) x\right) .
$$

These coefficients are clearly not polynomials in the parameters $\alpha^{\prime}, \beta^{\prime}$. However, we can remedy this problem by making the change of parameters $\alpha \stackrel{\text { def }}{=} \kappa\left(\alpha^{\prime}+\beta^{\prime}\right)$. Then (3.7) reduces to the form given in Theorem 3.1 for family 6 , and the coefficients (3.8) become polynomials (with nonnegative integer coefficients) jointly in $x$ and $\alpha^{\prime}, \beta^{\prime}, \gamma^{\prime}, \kappa$. A similar procedure handles families $4 \mathrm{a}$ and $4 \mathrm{~b}$. Once these changes of parameters have been performed, the coefficients $c_{i}$ are polynomials in $x$ and the chosen parameters:

F4a. $c_{2 k-1}=\left(\gamma^{\prime}+k \beta^{\prime}\right)(\kappa+x)$ and $c_{2 k}=k \beta^{\prime} x$.

F4b. $c_{2 k-1}=(\gamma+k \alpha)(1+\kappa x)$ and $c_{2 k}=k \alpha$.

F6. $c_{2 k-1}=\left[\gamma^{\prime}+k\left(\alpha^{\prime}+\beta^{\prime}\right)\right](\kappa+x)$ and $c_{2 k}=k\left(\alpha^{\prime}+\beta^{\prime}\right)(\kappa+x)$.

We thus again find a posteriori that the S-fraction (3.1) might also be valid when the chosen parameters (now including $\kappa$ ) are treated as indeterminates.

Of course, all the candidate families and their S-fractions are still at this point merely conjectures. We will prove them in Section 3.3, using some general results to be presented in Section 3.2.

\subsection{A "master S-fraction" for permutations}

Euler $[23 \text {, section } 21]^{3}$ showed that the generating function of the factorials can be represented as a beautiful S-fraction,

$$
\sum_{n=0}^{\infty} n ! t^{n}=\frac{1}{1-\frac{1 t}{1-\frac{1 t}{1-\frac{2 t}{1-\frac{2 t}{1-\cdots}}}}}
$$

\footnotetext{
${ }^{3}$ The paper [23], which is E247 in Eneström's [20] catalogue, was probably written circa 1746; it was presented to the St. Petersburg Academy in 1753, and published in 1760.
} 
with coefficients $c_{2 k-1}=c_{2 k}=k$. Inspired by (3.9), Sokal and Zeng [51] introduced the polynomials $\mathcal{P}_{n}(w, y, u, v)$ defined by the continued fraction

$$
\sum_{n=0}^{\infty} \mathcal{P}_{n}(w, y, u, v) t^{n}=\frac{1}{1-\frac{w t}{1-\frac{y t}{1-\frac{(w+u) t}{1-v) t}}}}
$$

with coefficients

$$
\begin{aligned}
c_{2 k-1} & =w+(k-1) u \\
c_{2 k} & =y+(k-1) v
\end{aligned}
$$

Clearly $\mathcal{P}_{n}(w, y, u, v)$ is a homogeneous polynomial of degree $n$; it therefore has three "truly independent" variables. Since $\mathcal{P}_{n}(1,1,1,1)=n$ !, which enumerates permutations of an $n$-element set, it is natural to expect that $\mathcal{P}_{n}(w, y, u, v)$ enumerates permutations of $[n]$ according to some natural trivariate statistic. Sokal and Zeng [51] gave two alternative versions of this trivariate statistic; here is one of them:

Theorem 3.2 (Sokal-Zeng). The polynomials $\mathcal{P}_{n}(w, y, u, v)$ defined by (3.10)/(3.11) have the combinatorial interpretation

$$
\mathcal{P}_{n}(w, y, u, v)=\sum_{\sigma \in \mathfrak{S}_{n}} w^{\operatorname{cyc}(\sigma)} y^{\operatorname{erec}(\sigma)} u^{n-\operatorname{exc}(\sigma)-\operatorname{cyc}(\sigma)} v^{\operatorname{exc}(\sigma)-\operatorname{erec}(\sigma)},
$$

where the sum runs over the set $\mathfrak{S}_{n}$ of permutations of $[n]$.

The permutation statistics appearing in (3.12) are defined as follows. Given a permutation $\sigma \in \mathfrak{S}_{n}$, an index $i \in[n]$ is called a

- record (rec) (or left-to-right maximum) if $\sigma(j)<\sigma(i)$ for all $j<i$ [note that index 1 is always a record];

- antirecord (arec) (or right-to-left minimum) if $\sigma(j)>\sigma(i)$ for all $j>i$ [note that index $n$ is always an antirecord];

- exclusive record (erec) if it is a record and not also an antirecord;

- exclusive antirecord (earec) if it is an antirecord and not also a record.

Also, an index $i \in[n]$ is called an excedance if $\sigma(i)>i$. The number of exclusive records, excedances and cycles of $\sigma$ are denoted by $\operatorname{erec}(\sigma), \operatorname{exc}(\sigma)$ and $\operatorname{cyc}(\sigma)$, respectively.

Remark. The foregoing quantities are a proper subset of a more elaborate classification of the permutations $\sigma \in \mathfrak{S}_{n}$ - the "record-and-cycle classification" into ten disjoint categories [51] — but we do not need this refinement here. 
For all the candidate families in Theorem 3.1, their conjectured S-fraction coefficients (see Section 3.1) belong to a very special subclass of Theorem 3.2, namely $c_{2 k} \propto k$, so that $v=y$ :

$$
\mathcal{P}_{n}(w, y, u, y)=\sum_{\sigma \in \mathfrak{S}_{n}} w^{\operatorname{cyc}(\sigma)} y^{\operatorname{exc}(\sigma)} u^{n-\operatorname{exc}(\sigma)-\operatorname{cyc}(\sigma)} .
$$

These latter polynomials have a nice explicit formula, as follows:

Proposition 3.3. The polynomial $\mathcal{P}_{n}(w, y, u, y)$ defined by (3.13) can be written as

$$
\begin{aligned}
\mathcal{P}_{n}(w, y, u, y) & =\sum_{r=0}^{n}\left\{\begin{array}{l}
n \\
r
\end{array}\right\}(y-u)^{n-r} \sum_{i=0}^{r}\left[\begin{array}{l}
r \\
i
\end{array}\right] w^{i} u^{r-i} \\
& =\sum_{r=0}^{n}\left\{\begin{array}{l}
n \\
r
\end{array}\right\}(y-u)^{n-r} \prod_{k=0}^{r-1}(w+k u),
\end{aligned}
$$

where $\left\{\begin{array}{l}n \\ k\end{array}\right\}$ denotes the number of partitions of an n-element set into $k$ nonempty blocks, and $\left[\begin{array}{l}n \\ k\end{array}\right]$ denotes the number of permutations of an n-element set with $k$ cycles.

Furthermore, the polynomials $\mathcal{P}_{n}(w, y, u, y)$ have the exponential generating function

$$
\sum_{n=0}^{\infty} \mathcal{P}_{n}(w, y, u, y) \frac{t^{n}}{n !}=\left(\frac{y-u}{y-u e^{(y-u) t}}\right)^{w / u}
$$

We shall prove the formulae $(3.14 \mathrm{a}, \mathrm{b})$ by making use of a result due to Ma [38, Corollary 2.3]:

Lemma 3.4 (Ma). For $n, i, \ell \geqslant 0$ with $i+\ell \leqslant n$, define

$$
A_{n}(i, \ell) \stackrel{\text { def }}{=} \mid\left\{\sigma \in \mathfrak{S}_{n}: \operatorname{cyc}(\sigma)=i \text { and } \operatorname{exc}(\sigma)=\ell\right\} \mid .
$$

Then

$$
A_{n}(i, \ell)=\sum_{r=i}^{n-\ell}(-1)^{n-r-\ell}\left\{\begin{array}{l}
n \\
r
\end{array}\right\}\left[\begin{array}{l}
r \\
i
\end{array}\right]\left(\begin{array}{c}
n-r \\
\ell
\end{array}\right) .
$$

And to get the exponential generating function (3.15), we shall use the following general result:

Lemma 3.5 (egf of a Stirling subset transform). Let $R$ be a commutative ring containing the rationals, let $\boldsymbol{a}=\left(a_{n}\right)_{n \geqslant 0}$ be a sequence in $R$, and let $x$ be an indeterminate; and define the sequence $\boldsymbol{b}=\left(b_{n}\right)_{n \geqslant 0}$ in $R[x]$ by

$$
b_{n}=\sum_{k=0}^{n}\left\{\begin{array}{l}
n \\
k
\end{array}\right\} a_{k} x^{n-k} .
$$

(We call this the " $x$-Stirling subset transform".) Then the exponential generating functions $A(t)=\sum_{n=0}^{\infty} a_{n} t^{n} / n$ ! and $B(t)=\sum_{n=0}^{\infty} b_{n} t^{n} / n$ ! are related by

$$
B(t)=A\left(\frac{e^{x t}-1}{x}\right) .
$$


Proof. We have

$$
\begin{aligned}
\sum_{n=0}^{\infty} a_{n} \frac{t^{n}}{n !} & =\sum_{n=0}^{\infty} \sum_{k=0}^{n}\left\{\begin{array}{l}
n \\
k
\end{array}\right\} a_{k} x^{n-k} \frac{t^{n}}{n !} \\
& =\sum_{k=0}^{\infty} a_{k} x^{-k} \sum_{n=k}^{\infty}\left\{\begin{array}{l}
n \\
k
\end{array}\right\} \frac{(x t)^{n}}{n !} \\
& =\sum_{k=0}^{\infty} a_{k} x^{-k} \frac{\left(e^{x t}-1\right)^{k}}{k !} \quad \text { by }[34, \text { eq. }(7.49)] \\
& =A\left(\frac{e^{x t}-1}{x}\right) .
\end{aligned}
$$

Proof of Proposition 3.3. Multiply (3.17) by $w^{i} y^{\ell} u^{n-i-\ell}$ and sum over $i$ and $\ell$. Using the well-known identities [34, eqs. (7.48)/(5.12)]

$$
\begin{aligned}
\sum_{i}\left[\begin{array}{l}
r \\
i
\end{array}\right] w^{i} u^{r-i} & =\prod_{k=0}^{r-1}(w+k u) \\
\sum_{\ell}\left(\begin{array}{c}
n-r \\
\ell
\end{array}\right) y^{\ell}(-u)^{n-r-\ell} & =(y-u)^{n-r}
\end{aligned}
$$

gives $(3.14 \mathrm{a}, \mathrm{b})$. Now use Lemma 3.5 with

$$
\sum_{r=0}^{\infty} \sum_{i=0}^{r}\left[\begin{array}{l}
r \\
i
\end{array}\right] w^{i} u^{r-i} \frac{t^{r}}{r !}=(1-u t)^{-w / u} \stackrel{\text { def }}{=} A(t)
$$

[34, eq. (7.55)] and $x=y-u$ to obtain (3.15).

Remarks. 1. Lemma 3.5 is a special case of the fundamental theorem of exponential Riordan arrays (FTERA) $[5,6]$.

2. Let us stress that this proof of Proposition 3.3 is based on the combinatorial definition (3.13) of the polynomials $\mathcal{P}_{n}(w, y, u, y)$; it makes no use of continued fractions or the Sokal-Zeng Theorem 3.2. On the other hand, Theorem 3.2 then tells us that the ogf of these polynomials has an S-fraction representation of the form (3.10) with $v=y$. Zeng [65] has shown that the J-fraction associated by contraction to this S-fraction (see Proposition 4.1 below) can alternatively be obtained directly from the egf (3.15) by the Stieltjes-Rogers addition-formula method.

Two particular cases of $(3.14) /(3.15)$ are of especial interest: 
1) When $u=y$, we obtain the homogenized Stirling cycle polynomials: $(3.13) /(3.14)$ reduce to

$$
\mathcal{P}_{n}(w, y, y, y)=\sum_{r=0}^{n}\left[\begin{array}{l}
n \\
r
\end{array}\right] w^{r} y^{n-r}=\prod_{k=0}^{n-1}(w+k y),
$$

the limit $u \rightarrow y$ of (3.15) gives the egf [34, eq. (7.55)]

$$
\sum_{n=0}^{\infty} \mathcal{P}_{n}(w, y, y, y) \frac{t^{n}}{n !}=(1-y t)^{-w / y}
$$

[cf. (3.23)], and the ogf has the S-fraction

$$
\sum_{n=0}^{\infty} \mathcal{P}_{n}(w, y, y, y) t^{n}=\frac{1}{1-\frac{w t}{1-\frac{y t}{1-\frac{(w+y) t}{1-\frac{2 y t}{1-\cdots}}}}}
$$

with coefficients $c_{2 k-1}=w+(k-1) y, c_{2 k}=k y$. This S-fraction was found by Euler $[23$, section 26] [24]. ${ }^{4}$

2) When $u=w$, we obtain the homogenized Eulerian polynomials: (3.13)/(3.14) reduce to

$$
\mathcal{P}_{n}(w, y, w, y)=\sum_{k=0}^{n}\left\langle\begin{array}{l}
n \\
k
\end{array}\right\rangle y^{k} w^{n-k}=\sum_{r=0}^{n} r !\left\{\begin{array}{l}
n \\
r
\end{array}\right\}(y-w)^{n-r} w^{r}
$$

where $\left\langle\begin{array}{l}n \\ k\end{array}\right\rangle$ denotes the number of permutations of $[n]$ with $k$ excedances, (3.15) gives the egf $[34$, eq. (7.56)]

$$
\sum_{n=0}^{\infty} \mathcal{P}_{n}(w, y, w, y) \frac{t^{n}}{n !}=\frac{y-w}{y-w e^{(y-w) t}},
$$

and the ogf has the S-fraction

$$
\sum_{n=0}^{\infty} \mathcal{P}_{n}(w, y, y, y) t^{n}=\frac{1}{1-\frac{w t}{1-\frac{y t}{1-\frac{2 w t}{1-\frac{2 y t}{1-\cdots}}}}}
$$

\footnotetext{
${ }^{4}$ The paper [24], which is E616 in Eneström's [20] catalogue, was apparently presented to the St. Petersburg Academy in 1776, and published posthumously in 1788.
} 
with coefficients $c_{2 k-1}=k w, c_{2 k}=k y$. This S-fraction was found by Stieltjes [56, section 79]. ${ }^{5}$ The equality of the last two terms in (3.27) is a well-known identity [34, eqns. $(6.39) /(6.40)]$ that relates the Eulerian polynomials to the ordered Bell polynomials. $^{6}$

Remarks. The polynomials (3.14) apparently first appeared in the work of Carlitz [9]. (He considered the case $u=1$, but this is equivalent by homogenization to the general case.) More specifically, Carlitz [9, p. 422] (see also Dillon and Roselle [13]) showed that the generalized Eulerian polynomials $A_{n}(y, w)$ defined by the exponential generating function

$$
\left(\frac{1-y}{e^{(y-1) t}-y}\right)^{w}=\sum_{n=0}^{\infty} A_{n}(y, w) \frac{t^{n}}{n !}
$$

have the explicit expression

$$
A_{n}(y, w)=\sum_{r=0}^{n}\left\{\begin{array}{l}
n \\
r
\end{array}\right\}(y-1)^{n-r} \prod_{k=0}^{r-1}(w+k) .
$$

(We proved this in reverse by using Lemma 3.5.) The formula (3.17) for the coefficients of the polynomials (3.31) can also be found in Dillon and Roselle [13, eqns. $(1.3) /(3.3) /(3.5)]$.

Furthermore, Dillon and Roselle [13, section 5] showed that $A_{n}(y, w)$ enumerates permutations of $[n]$ with a weight $y$ for each descent [i.e. each index $i \in[n-1]$ such that $\sigma(i)>\sigma(i+1)]$ and a weight $w$ for each record. And by Foata's fundamental transformation, this is equivalent to enumerating permutations of $[n]$ with a weight $y$ for each excedance and $w$ for each cycle. ${ }^{7}$ So this chain of reasoning gives an alternate proof of the equivalences between (3.13), (3.14) and (3.15).

\footnotetext{
${ }^{5}$ Stieltjes does not specifically mention the Eulerian polynomials, but he does state that the continued fraction is the formal Laplace transform of $(1-y) /\left(e^{t(y-1)}-y\right)$, which is well known to be the exponential generating function of the Eulerian polynomials [cf. (3.28) with $w=1$ ]. Stieltjes also refrains from showing the proof: "Pour abréger, je supprime toujours les artifices qu'il faut employer pour obtenir la transformation de l'intégrale définie en fraction continue" (!). But a proof is sketched, albeit also without much explanation, in the book of Wall [63, pp. 207-208]. The J-fraction corresponding to the contraction (see Proposition 4.1 below) of this S-fraction was proven, by combinatorial methods, by Flajolet [27, Theorem 3B(ii) with a slight typographical error]. Also, Dumont [15, Propositions 2 and 7] gave a direct combinatorial proof of the S-fraction, based on an interpretation of the Eulerian polynomials in terms of "bipartite involutions of $[2 n]$ " and a bijection of these onto Dyck paths.

${ }^{6}$ The ordered Bell polynomials appear already (albeit without the combinatorial interpretation) in Euler's book Foundations of Differential Calculus, with Applications to Finite Analysis and Series, first published in 1755 [22, paragraph 172]. This book is E212 in Eneström's [20] catalogue. Furthermore, the identity (3.27) appears already there [22, paragraphs 172 and 173]; it was rediscovered a century-and-ahalf later by Frobenius [30]. See also [28, pp. 150-151] for a simple bijective proof.

${ }^{7}$ We use Foata's fundamental transformation [29, section I.3] in the following form [55, pp. 17-18]: Given a permutation $\sigma \in \mathfrak{S}_{n}$, we write $\sigma$ in disjoint cycle notation with the convention that (a) each cycle is written with its largest element (the cycle maximum) first, and (b) the cycles are written in increasing order of their largest element; we then erase the parentheses and call the resulting word $\widehat{\sigma}$. The map $\sigma \mapsto \widehat{\sigma}$ is a bijection, because the permutation $\sigma$ can be uniquely recovered from $\widehat{\sigma}$ by inserting a left parenthesis preceding each record and a right parenthesis where appropriate (i.e. before every internal left parenthesis and at the end). There is now a one-to-one correspondence between cycle maxima in $\sigma$
} 
On the other hand, Stieltjes [56, section 81] observed that the continued fraction (3.10) with $v=y$ and $u=1$ is the formal Laplace transform of the exponential generating function (3.30). ${ }^{8}$ Combining this result with the just-mentioned combinatorial interpretation of $A_{n}(y, w)$ in terms of excedances and cycles [29, section IV.3] proves the $v=y$ special case of the Sokal-Zeng Theorem 3.2. (By contrast, the proof of Sokal and Zeng [51] is purely combinatorial, and makes no use of exponential generating functions.)

See also $[8,10,36,38,47]$ for some later work on these polynomials.

\subsection{Proof that the candidate families have an S-fraction representation}

We will now complete the proof of Theorem 3.1 by showing that each of the ten families indeed has an S-fraction representation with the conjectured coefficients $\boldsymbol{c}$. This section is organized as follows: For each family, we will state the main result and give its proof; then we will describe some additional properties of the given family and mention some special cases of combinatorial interest. The families are grouped by duality; two families related by duality will have essentially identical proofs, with $c_{i}(x) \rightarrow x c_{i}(1 / x)$. Each proof will be based on identifying the exponential generating function (1.4) as a special case of (3.15); it will then follow from Theorem 3.2 and Proposition 3.3 that the corresponding ordinary generating function (1.3) is given by the S-fraction (3.12) with $v=y$ and suitable values for $w, u, y$.

\subsubsection{Families $1 \mathrm{a}$ and $1 \mathrm{~b}$}

Proposition 3.6 (S-fraction for families $1 \mathrm{a}$ and $1 \mathrm{~b})$. The ogf $f(x, t ; \boldsymbol{\mu})$ for the recurrence (1.1) with $\boldsymbol{\mu}=\left(0, \beta, 0, \alpha^{\prime},-\alpha^{\prime}, \gamma^{\prime}\right)$ has an $S$-type continued fraction representation in the ring $\mathbb{Z}\left[x, \beta, \alpha^{\prime}, \gamma^{\prime}\right][[t]]$ with coefficients

$$
c_{2 k-1}=\left[\gamma^{\prime}+(k-1) \alpha^{\prime}\right] x, \quad c_{2 k}=k \beta .
$$

Similarly, the ogf $f(x, t ; \boldsymbol{\mu})$ for the recurrence $(1.1)$ with $\boldsymbol{\mu}=\left(0, \beta, \gamma, \alpha^{\prime},-\alpha^{\prime}, 0\right)$ has an $S$-type continued fraction representation in the ring $\mathbb{Z}\left[x, \beta, \gamma, \alpha^{\prime}\right][[t]]$ with coefficients

$$
c_{2 k-1}=\gamma+(k-1) \beta, \quad c_{2 k}=k \alpha^{\prime} x .
$$

Family 1a: $\mu=\left(0, \beta, 0 ; \alpha^{\prime},-\alpha^{\prime}, \gamma^{\prime}\right)$

This is Spivey's [52] case (S3) $\alpha / \beta=\alpha^{\prime} / \beta^{\prime}+1$ specialized to $\alpha=\gamma=0$. Its egf can be computed from $[2$, eq. (A2)]:

$$
F(x, t)=\left[\frac{\beta-\alpha^{\prime} x e^{\left(\beta-\alpha^{\prime} x\right) t}}{\beta-\alpha^{\prime} x}\right]^{-\gamma^{\prime} / \alpha^{\prime}} .
$$

and records in $\widehat{\sigma}$, and between anti-excedances in $\sigma$ and descents in $\widehat{\sigma}$. Finally, the bijection $\sigma \mapsto R \circ \sigma \circ R$ where $R(i)=n+1-i$ interchanges anti-excedances and excedances while preserving the number of cycles. This proves the equivalence asserted in the text.

${ }^{8}$ Here too, Stieltjes refrained from showing the proof: see footnote 5 . 
This is the special case of (3.15) with $w=\gamma^{\prime} x, u=\alpha^{\prime} x, y=\beta$, so the ogf is given by the S-fraction (3.11) with $w=\gamma^{\prime} x, u=\alpha^{\prime} x, y=v=\beta$, exactly as stated in (3.32). This completes the proof for family 1a.

Family 1b: $\mu=\left(0, \beta, \gamma, \alpha^{\prime},-\alpha^{\prime}, 0\right)$

This is Spivey's [52] case (S3) $\alpha / \beta=\alpha^{\prime} / \beta^{\prime}+1$ specialized now to $\alpha=\gamma^{\prime}=0$. Its egf is $[2$, eq. (A2)]

$$
F(x, t)=\left[\frac{\alpha^{\prime} x-\beta e^{\left(\alpha^{\prime} x-\beta\right) t}}{\alpha^{\prime} x-\beta}\right]^{\gamma / \beta} .
$$

This is the special case of (3.15) with $w=\gamma, u=\beta, y=\alpha^{\prime} x$, so the ogf is given by the S-fraction (3.11) with $w=\gamma, u=\beta, y=v=\alpha^{\prime} x$, exactly as stated in (3.33). This completes the proof for family $1 \mathrm{~b}$.

Of course, this result can also be obtained by applying duality to family 1a. Since the dual of $\boldsymbol{\mu}=\left(0, \beta, 0, \alpha^{\prime},-\alpha^{\prime}, \gamma^{\prime}\right)$ is $D \boldsymbol{\mu}=\left(0, \alpha^{\prime}, \gamma^{\prime}, \beta,-\beta, 0\right)$, we obtain family $1 \mathrm{~b}$ from the dual of family 1a by applying the map $\left(\alpha^{\prime}, \gamma^{\prime}, \beta\right) \mapsto\left(\beta, \gamma, \alpha^{\prime}\right)$.

\section{Particular cases}

These two families contain several specific cases of combinatorial interest:

- The Stirling subset numbers $\left\{\begin{array}{l}n \\ k\end{array}\right\}$ have $\boldsymbol{\mu}=(0,1,0,0,0,1)$ and belong to family 1 a. The S-fraction for the ogf of the Bell polynomials $B_{n}(x)=\sum_{k=0}^{n}\left\{\begin{array}{l}n \\ k\end{array}\right\} x^{k}$, with coefficients $c_{2 k-1}=x$ and $c_{2 k}=k$, is well known. ${ }^{9}$

- The generalized $(s, 0)$-Eulerian numbers $[3]\left\langle\begin{array}{l}n \\ k\end{array}\right\rangle_{(s, 0)}$ have $\boldsymbol{\mu}=(0,1, s, 1,-1,0)$ with $s \in \mathbb{N}$ and belong to family $1 \mathrm{~b}$. The case $s=1$ corresponds to the Eulerian numbers $\left\langle\begin{array}{l}n \\ k\end{array}\right\rangle$ with the Graham-Knuth-Patashnik indexing [34, section 6.2] [41, A173018].

- The generalized $(0, t)$-Eulerian numbers $[3]\left\langle\begin{array}{l}n \\ k\end{array}\right\rangle_{(0, t)}$ have $\boldsymbol{\mu}=(0,1,0,1,-1, t)$ with $t \in \mathbb{N}$ and belong to family 1a. The case $t=1$ corresponds to the Eulerian numbers with the traditional indexing $\left\langle\begin{array}{l}n \\ k\end{array}\right\rangle_{(0,1)}=\left\langle\begin{array}{c}n \\ k-1\end{array}\right\rangle$ for $n \geqslant 1$ [41, A008292].

\subsubsection{Families $2 \mathrm{a}$ and $2 \mathrm{~b}$}

Proposition 3.7 (S-fraction for families $2 \mathrm{a}$ and $2 \mathrm{~b}$ ). The ogf $f(x, t ; \boldsymbol{\mu})$ for the recurrence (1.1) with $\boldsymbol{\mu}=\left(\alpha,-\alpha,-\alpha, \alpha^{\prime}, \beta^{\prime}, \gamma^{\prime}\right)$ has an S-type continued fraction representation in the ring $\mathbb{Z}\left[x ; \alpha, \alpha^{\prime}, \beta^{\prime}, \gamma^{\prime}\right][[t]]$ with coefficients

$$
c_{2 k-1}=\left[\gamma^{\prime}+k\left(\alpha^{\prime}+\beta^{\prime}\right)\right] x, \quad c_{2 k}=k\left(\alpha^{\prime}+\beta^{\prime}\right) x .
$$

\footnotetext{
${ }^{9}$ Flajolet [27, Theorem 2(ib)] found a J-type continued fraction that is equivalent by contraction (see Proposition 4.1 below) to this S-fraction. Later, Dumont [16] found the S-fraction directly by a functionalequation method, and Zeng [66, Lemma 3] used this same method to find two $q$-generalizations of the S-fraction.
} 
Similarly, the ogf $f(x, t ; \boldsymbol{\mu})$ for the recurrence (1.1) with $\boldsymbol{\mu}=\left(\alpha, \beta, \gamma, 0, \beta^{\prime},-\beta^{\prime}\right)$ has an $S$-type continued fraction representation in the ring $\mathbb{Z}\left[x ; \alpha, \beta, \gamma, \beta^{\prime}\right][[t]]$ with coefficients

$$
c_{2 k-1}=\gamma+k \alpha, \quad c_{2 k}=k \alpha .
$$

Before proceeding further, let us observe that the coefficients (3.36) for family 2a do not depend on $\alpha$, and moreover they depend on $\alpha^{\prime}$ and $\beta^{\prime}$ only via their sum. Similarly, the coefficients (3.37) for family $2 \mathrm{~b}$ do not depend on $\beta$ or $\beta^{\prime}$. These cases illustrate the parametric ambiguities discussed in [2, Section 3], in which the map $\boldsymbol{\mu} \mapsto \boldsymbol{T}(\boldsymbol{\mu})$ fails to be injective. So families $2 \mathrm{a}$ and $2 \mathrm{~b}$, which appear to be four-dimensional, are in fact only two-dimensional.

This degeneracy also implies that family $2 \mathrm{a}$, which is defined by $\boldsymbol{\mu}=(\alpha,-\alpha,-\alpha$, $\left.\alpha^{\prime}, \beta^{\prime}, \gamma^{\prime}\right)$, in fact has the same matrix $\boldsymbol{T}(\boldsymbol{\mu})$ as $\boldsymbol{\mu}^{\prime}=\left(0,0,0,0, \alpha^{\prime}+\beta^{\prime}, \gamma^{\prime}\right)$, which is a special case of family 3a. The S-fraction (3.36) for family $2 \mathrm{a}$ is thus obtained from the S-fraction (3.45) for family 3a by making the replacements $\beta \rightarrow 0$ and $\beta^{\prime} \rightarrow \alpha^{\prime}+\beta^{\prime}$. Similarly, family $2 \mathrm{~b}$, which is defined by $\boldsymbol{\mu}=\left(\alpha, \beta, \gamma, 0, \beta^{\prime},-\beta^{\prime}\right)$, has the same matrix $\boldsymbol{T}(\boldsymbol{\mu})$ as $\boldsymbol{\mu}^{\prime}=(\alpha,-\alpha, \gamma, 0,0,0)$, which is a special case of family $3 \mathrm{~b}$. So the S-fraction (3.37) for family $2 \mathrm{~b}$ is obtained from the S-fraction (3.46) for family $3 \mathrm{~b}$ by making the specialization $\alpha^{\prime}=0$. Consequently, families $2 \mathrm{a}$ and $2 \mathrm{~b}$ are redundant if we work at the level of the matrices $\boldsymbol{T}(\boldsymbol{\mu})$ rather than the parameters $\boldsymbol{\mu}$.

In fact, both of these families are also degenerate in a further sense: in family 2 a we have $T(n, k)=0$ whenever $k \neq n$, and in family $2 \mathrm{~b}$ we have $T(n, k)=0$ whenever $k \neq 0$. From the recurrence (1.1) one easily gets for family $2 \mathrm{a}$

$$
T(n, k)=\delta_{k n} \prod_{j=1}^{n}\left[\gamma^{\prime}+j\left(\alpha^{\prime}+\beta^{\prime}\right)\right]
$$

and hence

$$
P_{n}(x)=x^{n} \prod_{j=1}^{n}\left[\gamma^{\prime}+j\left(\alpha^{\prime}+\beta^{\prime}\right)\right]
$$

and for family $2 b$

$$
T(n, k)=\delta_{k 0} \prod_{j=1}^{n}(\gamma+j \alpha)
$$

and hence

$$
P_{n}(x)=\prod_{j=1}^{n}(\gamma+j \alpha) .
$$

In these formulae we see explicitly the parametric ambiguities mentioned above. The S-fractions (3.36) and (3.37) are then simply the S-fraction (3.26) for the homogenized Stirling cycle polynomials with the variables $w$ and $y$ in that formula replaced by suitable linear combinations of the parameters $\boldsymbol{\mu}$. 
Alternatively, we can deduce the S-fractions directly from the exponential generating functions. Family 2a is Spivey's [52] case (S1) $\beta=-\alpha$ specialized to $\gamma=-\alpha$, and its egf is $[2$, eq. (A4)

$$
F(x, t)=\left[1-\left(\alpha^{\prime}+\beta^{\prime}\right) x t\right]^{-\left(\alpha^{\prime}+\beta^{\prime}+\gamma^{\prime}\right) /\left(\alpha^{\prime}+\beta^{\prime}\right)} .
$$

This is the special case of (3.25) with $w=\left(\alpha^{\prime}+\beta^{\prime}+\gamma^{\prime}\right) x, y=\left(\alpha^{\prime}+\beta^{\prime}\right) x$, and inserting these parameters into (3.26) gives (3.36). Family $2 \mathrm{~b}$ is Neuwirth's [40] case $\alpha^{\prime}=0$ specialized to $\gamma^{\prime}=-\beta^{\prime}$, and its egf is $[2$, eq. (A8)]

$$
F(x, t)=(1-\alpha t)^{-(\alpha+\gamma) / \alpha} .
$$

This is the special case of (3.25) with $w=\alpha+\gamma, y=\alpha$, and inserting these parameters into (3.26) gives (3.37).

\section{Particular cases}

These two families contain several specific cases of combinatorial interest:

- Multifactorials: They appear in both families. To make the story short, let us consider family $2 \mathrm{~b}$. It is obvious that $\boldsymbol{\mu}=(0,0,1,0,0,0)$ leads to $T(n, k)=\delta_{k 0}$. If we apply Corollary 2.2(c) with $\kappa=\nu$ and $\lambda=-\rho$, we get that the triangular-array entries

$$
T^{\prime}(n, k)=\left(\prod_{j=0}^{n-1}(n \nu-\rho-j \nu)\right) \delta_{k 0}
$$

satisfy the GKP recurrence with $\boldsymbol{\mu}^{\prime}=(\nu, 0,-\rho, 0,0,0)$. For instance:

○ If $\nu=1$ and $\rho=0$, then $\boldsymbol{\mu}^{\prime}=(1,0,0,0,0,0)$ leads to the factorials $T^{\prime}(n, k)=$ $n ! \delta_{k 0}$. In this case, we recover Euler's S-fraction (3.9).

○ If $\nu=2$ and $\rho=-1$, then $\boldsymbol{\mu}^{\prime}=(2,0,-1,0,0,0)$ leads to the semi-factorials $T^{\prime}(n, k)=(2 n-1) ! ! \delta_{k 0}$, or the double factorial of odd numbers [41, A001147]. The corresponding S-fraction was also found by Euler [23, section 29].

○ If $\nu=3$ and $\rho=-1$, then $\boldsymbol{\mu}^{\prime}=(3,0,-1,0,0,0)$ leads to the triple factorials $T^{\prime}(n, k)=(3 n-1) ! ! ! \delta_{k 0}[41, \mathrm{~A} 007661]$.

o If $\nu=4$ and $\rho=-1$, then $\boldsymbol{\mu}^{\prime}=(4,0,-1,0,0,0)$ leads to the quadruple factorials $T^{\prime}(n, k)=(4 n-1) ! ! ! ! \delta_{k 0}[41, \mathrm{~A} 007662]$.

○ In general, if $\nu \geqslant 2$ is an integer, then $\boldsymbol{\mu}^{\prime}=(\nu, 0,-1,0,0,0)$ leads to the $\nu$-th factorials $T^{\prime}(n, k)=\prod_{j=0}^{n-1}(n \nu-j \nu-1) \delta_{k 0}$.

- $\boldsymbol{\mu}=(0,1, s, 0,1,-1)$ leads to $T(n, k)=\delta_{k 0} s^{n}$. These numbers are the inverse pairs of the generalized Eulerian numbers $\left\langle\begin{array}{l}n \\ k\end{array}\right\rangle_{(s,-s)}[3]$ given by $\boldsymbol{\mu}=(0,1, s, 1,-1,-s)$.

(See the end of Section 3.3.6.) 


\subsubsection{Families $3 \mathrm{a}$ and $3 \mathrm{~b}$}

Proposition 3.8 (S-fraction for families $3 \mathrm{a}$ and $3 \mathrm{~b})$. The ogf $f(x, t ; \boldsymbol{\mu})$ for the recurrence (1.1) with $\boldsymbol{\mu}=\left(0, \beta, 0,0, \beta^{\prime}, \gamma^{\prime}\right)$ has an $S$-type continued fraction representation in the ring $\mathbb{Z}\left[x, \beta, \beta^{\prime}, \gamma^{\prime}\right][[t]]$ with coefficients

$$
c_{2 k-1}=\left(\gamma^{\prime}+k \beta^{\prime}\right) x, \quad c_{2 k}=k\left(\beta+\beta^{\prime} x\right) .
$$

Similarly, the ogf $f(x, t ; \boldsymbol{\mu})$ for the recurrence $(1.1)$ with $\boldsymbol{\mu}=\left(\alpha,-\alpha, \gamma, \alpha^{\prime},-\alpha^{\prime}, 0\right)$ has an $S$-type continued fraction representation in the ring $\mathbb{Z}\left[x, \alpha, \gamma, \alpha^{\prime}\right][[t]]$ with coefficients

$$
c_{2 k-1}=\gamma+k \alpha, \quad c_{2 k}=k\left(\alpha+\alpha^{\prime} x\right) \text {. }
$$

Family 3a: $\mu=\left(0, \beta, 0 ; 0, \beta^{\prime}, \gamma^{\prime}\right)$

Family 3a is Neuwirth's [40] case $\alpha^{\prime}=0$ specialized to $\alpha=\gamma=0$. Its egf is [2, eq. (A8)]

$$
F(x, t)=\left[1+\frac{\beta^{\prime} x}{\beta}\left(1-e^{\beta t}\right)\right]^{-\left(\beta^{\prime}+\gamma^{\prime}\right) / \beta^{\prime}} .
$$

This is the special case of (3.15) with $w=\left(\beta^{\prime}+\gamma^{\prime}\right) x, u=\beta^{\prime} x, y=\beta+\beta^{\prime} x$, so the ogf is given by the $\mathrm{S}$-fraction with coefficients $(3.45)$.

Family 3b: $\mu=\left(\alpha,-\alpha, \gamma, \alpha^{\prime},-\alpha^{\prime}, 0\right)$

Family $3 \mathrm{~b}$ is Spivey's [52] case (S1) $\beta=-\alpha$ specialized to $\beta^{\prime}=-\alpha^{\prime}$ and $\gamma^{\prime}=0$, and its egf is $[2$, eq. (A4)]

$$
F(x, t)=\left[1+\frac{\alpha}{\alpha^{\prime} x}\left(1-e^{\alpha^{\prime} x t}\right)\right]^{-(\alpha+\gamma) / \alpha} .
$$

This is the special case of (3.15) with $w=\alpha+\gamma, u=\alpha, y=\alpha+\alpha^{\prime} x$, so the ogf is given by the S-fraction with coefficients (3.46). Of course, this result can also be obtained by applying duality to family $3 \mathrm{a}$.

\section{Particular cases}

These two families contain several interesting special cases, although some of them have already appeared in the previous two subsections:

- The Stirling subset numbers $\left\{\begin{array}{l}n \\ k\end{array}\right\}$ have $\boldsymbol{\mu}=(0,1,0,0,0,1)$ and belong to family 3a (as well as 1a).

- The ordered Stirling subset numbers (also called surjective numbers [26]) $k !\left\{\begin{array}{l}n \\ k\end{array}\right\}$ have $\boldsymbol{\mu}=(0,1,0,0,1,0)$ and belong to family $3 \mathrm{a}$. 
- Multifactorials: we now apply Corollary 2.2(b) to $\boldsymbol{\mu}=(0,0,1,0,0,0)$ with $\kappa=\nu$ and $\lambda=-\rho$. Then, the numbers

$$
T^{\prime}(n, k)=\left(\prod_{j=1}^{n}(j \nu-\rho)\right) \delta_{k 0}
$$

satisfy the GKP recurrence with $\boldsymbol{\mu}^{\prime}=(\nu,-\nu,-\rho, 0,0,0)$ which belongs to family $3 \mathrm{~b}$. By choosing $\nu$ and $\rho$ in the same way as we did at the end of Section 3.3.2, we obtain factorials, double factorials, triple factorials, etc.

\subsubsection{Families $4 \mathrm{a}$ and $4 \mathrm{~b}$}

Proposition 3.9 (S-fraction for families $4 \mathrm{a}$ and $4 \mathrm{~b})$. The ogf $f(x, t ; \boldsymbol{\mu})$ for the recurrence (1.1) with $\boldsymbol{\mu}=\left(0, \kappa \beta^{\prime}, \kappa\left(\beta^{\prime}+\gamma^{\prime}\right), 0, \beta^{\prime}, \gamma^{\prime}\right)$ has an $S$-type continued fraction representation in the ring $\mathbb{Z}\left[x ; \beta^{\prime}, \gamma^{\prime}, \kappa\right][[t]]$ with coefficients

$$
c_{2 k-1}=\left(\gamma^{\prime}+k \beta^{\prime}\right)(\kappa+x), \quad c_{2 k}=k \beta^{\prime} x .
$$

Similarly, the ogf $f(x, t ; \boldsymbol{\mu})$ for the recurrence $(1.1)$ with $\boldsymbol{\mu}=(\alpha,-\alpha, \gamma, \kappa \alpha,-\kappa \alpha, \kappa(\alpha+\gamma))$ has an $S$-type continued fraction representation in the ring $\mathbb{Z}[x ; \alpha, \gamma, \kappa][[t]]$ with coefficients

$$
c_{2 k-1}=(\gamma+k \alpha)(1+\kappa x), \quad c_{2 k}=k \alpha .
$$

Family 4a: $\mu=\left(0, \kappa \beta^{\prime}, \kappa\left(\beta^{\prime}+\gamma^{\prime}\right), 0, \beta^{\prime}, \gamma^{\prime}\right)$

Family 4a is Neuwirth's [40] case $\alpha^{\prime}=0$ specialized to $\alpha=0$ and $\beta / \beta^{\prime}=(\gamma-\beta) / \gamma^{\prime}$. Its egf is $[2$, eq. (A8)]

$$
F(x, t)=\left[1-\frac{\kappa+x}{\kappa}\left(1-e^{-\kappa \beta^{\prime} t}\right)\right]^{-\left(\beta^{\prime}+\gamma^{\prime}\right) / \beta^{\prime}} .
$$

This is the special case of (3.15) with $w=\left(\beta^{\prime}+\gamma^{\prime}\right)(\kappa+x), u=\beta^{\prime}(\kappa+x), y=\beta^{\prime} x$, so the ogf is given by the S-fraction with coefficients (3.50).

Family $4 \mathrm{~b}: \mu=(\alpha,-\alpha, \gamma, \kappa \alpha,-\kappa \alpha, \kappa(\alpha+\gamma))$

Family $4 \mathrm{~b}$ is Spivey's [52] case (S1) $\beta=-\alpha$ specialized to $\beta^{\prime}=-\alpha^{\prime}$ and $\gamma^{\prime} / \alpha^{\prime}=$ $1+\gamma / \alpha$, and its egf is [2, eq. (A4)]

$$
F(x, t)=\left[1-\frac{1+\kappa x}{\kappa x}\left(1-e^{-\kappa \alpha x t}\right)\right]^{-(\alpha+\gamma) / \alpha} .
$$

This is the special case of $(3.15)$ with $w=(\alpha+\gamma)(1+\kappa x), u=\alpha(1+\kappa x), y=\alpha$, so the ogf is given by the S-fraction with coefficients (3.51). 


\section{Particular cases}

- Family 4a with $\gamma^{\prime}=0, \beta=1$, i.e. $\boldsymbol{\mu}=(0,1,1,0,1,0)$, yields a sequence [41, A028246] closely related to the ordered Stirling subset numbers:

$$
T(n, k)=k !\left\{\begin{array}{l}
n+1 \\
k+1
\end{array}\right\} .
$$

Note that these numbers and the Eulerian numbers $\left\langle\begin{array}{l}n \\ k\end{array}\right\rangle$ with $\boldsymbol{\mu}=(0,1,1,1,-1,0)$ (cf. family $1 \mathrm{~b}$ ), are related by the Riordan involution $R$.

\subsubsection{Family 5}

This self-dual family is given by $\boldsymbol{\mu}=\left(\alpha, 0, \gamma, \alpha^{\prime}, 0, \gamma^{\prime}\right)$. It corresponds to the case of the GKP recurrence (1.1) in which the coefficients depend only on $n$, and is Spivey's [52] case (S2) $\beta=\beta^{\prime}=0$. It is the Type-IV case of Problem 1.1 as defined in Ref. [2], and we can read all the relevant information from [2, eqs. (2.15), (A20), (A21)]:

$$
\begin{aligned}
F(x, t) & =\left[1-\left(\alpha+\alpha^{\prime} x\right) t\right]^{-\left[(\alpha+\gamma)+\left(\alpha^{\prime}+\gamma^{\prime}\right) x\right] /\left(\alpha+\alpha^{\prime} x\right)} \\
P_{n}(x) & =\prod_{k=1}^{n}\left[\left(\gamma+\gamma^{\prime} x\right)+k\left(\alpha+\alpha^{\prime} x\right)\right] \\
T(n, k) & =\sum_{t=0}^{n} \sum_{s=0}^{t}\left[\begin{array}{l}
n \\
t
\end{array}\right]\left(\begin{array}{l}
t \\
s
\end{array}\right)\left(\begin{array}{l}
n-t \\
k-s
\end{array}\right)(\alpha+\gamma)^{t-s}\left(\alpha^{\prime}+\gamma^{\prime}\right)^{s} \alpha^{n-k+s-t}\left(\alpha^{\prime}\right)^{k-s}
\end{aligned}
$$

So these polynomials are just translates and rescalings of the homogenized Stirling cycle polynomials (3.24). Using (3.26) with $w=(\alpha+\gamma)+\left(\alpha^{\prime}+\gamma^{\prime}\right) x$ and $y=\left(\alpha+\alpha^{\prime} x\right)$, we obtain:

Proposition 3.10 (S-fraction for family 5$)$. The ogf $f(x, t ; \boldsymbol{\mu})$ for the recurrence (1.1) with $\boldsymbol{\mu}=\left(\alpha, 0, \gamma, \alpha^{\prime}, 0, \gamma^{\prime}\right)$ has an $S$-type continued fraction representation in the ring $\mathbb{Z}\left[x ; \alpha, \gamma, \alpha^{\prime}, \gamma^{\prime}\right][[t]]$ with coefficients

$$
c_{2 k-1}=\left(\gamma+\gamma^{\prime} x\right)+k\left(\alpha+\alpha^{\prime} x\right), \quad c_{2 k}=k\left(\alpha+\alpha^{\prime} x\right) .
$$

As previously mentioned, family 5 is self-dual. Duality (2.5) acts on family 5 by interchanging $(\alpha, \gamma) \leftrightarrow\left(\alpha^{\prime}, \gamma^{\prime}\right)$.

\section{Particular cases}

- The binomial coefficients $\left(\begin{array}{l}n \\ k\end{array}\right)$ have $\boldsymbol{\mu}=(0,0,1,0,0,1)$. In this case the ogf is a rational function $f(x, t)=1 /[1-(1+x) t]$, and the S-fraction terminates at the first level (that is, $c_{2}=0$ ).

- The Stirling cycle numbers $\left[\begin{array}{l}n \\ k\end{array}\right]$ correspond to $\boldsymbol{\mu}=(1,0,-1,0,0,1)$.

- The reversed Stirling cycle numbers $\left[\begin{array}{c}n \\ n-k\end{array}\right]$ correspond to $\boldsymbol{\mu}=(0,0,1,1,0,-1)$. 


\subsubsection{Family 6}

This self-dual family is given by $\boldsymbol{\mu}=\left(\kappa\left(\alpha^{\prime}+\beta^{\prime}\right), \kappa \beta^{\prime}, \kappa \gamma^{\prime}, \alpha^{\prime}, \beta^{\prime}, \gamma^{\prime}\right)$. It is Spivey's [52] case (S3) $\alpha / \beta=\alpha^{\prime} / \beta^{\prime}+1$ specialized to $\beta / \beta^{\prime}=\gamma / \gamma^{\prime}$. We can deduce all the relevant information from $[2$, eq. (A2)]:

$$
\begin{aligned}
F(x, t) & =\left[1-\left(\alpha^{\prime}+\beta^{\prime}\right)(\kappa+x) t\right]^{-\left(\alpha^{\prime}+\beta^{\prime}+\gamma^{\prime}\right) /\left(\alpha^{\prime}+\beta^{\prime}\right)} \\
P_{n}(x) & =(\kappa+x)^{n} \prod_{j=1}^{n}\left[\gamma^{\prime}+j\left(\alpha^{\prime}+\beta^{\prime}\right)\right] \\
T(n, k) & =\kappa^{n-k}\left(\begin{array}{l}
n \\
k
\end{array}\right) \prod_{j=1}^{n}\left[\gamma^{\prime}+j\left(\alpha^{\prime}+\beta^{\prime}\right)\right]
\end{aligned}
$$

Like family 5 , these polynomials are translates and rescalings of the homogenized Stirling cycle polynomials (3.24), but now in the parameters rather than in $x$; the $x$-dependence is a trivial power $(\kappa+x)^{n}$. Using (3.26) with $w=\left(\alpha^{\prime}+\beta^{\prime}+\gamma^{\prime}\right)(\kappa+x)$ and $y=\left(\alpha^{\prime}+\beta^{\prime}\right)(\kappa+x)$ we obtain:

Proposition 3.11 (S-fraction for family 6$)$. The ogf $f(x, t ; \boldsymbol{\mu})$ for the recurrence (1.1) with $\boldsymbol{\mu}=\left(\kappa\left(\alpha^{\prime}+\beta^{\prime}\right), \kappa \beta^{\prime}, \kappa \gamma^{\prime}, \alpha^{\prime}, \beta^{\prime}, \gamma^{\prime}\right)$. has an S-type continued fraction representation in the ring $\mathbb{Z}\left[x, \alpha^{\prime}, \beta^{\prime}, \gamma^{\prime}, \kappa\right][[t]]$ with coefficients

$$
c_{2 k-1}=\left[\gamma^{\prime}+k\left(\alpha^{\prime}+\beta^{\prime}\right)\right](\kappa+x), \quad c_{2 k}=k\left(\alpha^{\prime}+\beta^{\prime}\right)(\kappa+x)
$$

Please note that the coefficients (3.62) for family 6 depend on $\alpha^{\prime}$ and $\beta^{\prime}$ only via their sum. So family 6 , which appears to be four-dimensional, is in fact only three-dimensional. Moreover, the parameter $\kappa$ acts only by translation of $x$. In fact, family 6 is derived from family 2 a by the substitution $x \mapsto x+\kappa$. It follows that the matrix $\boldsymbol{T}(\boldsymbol{\mu})$ for family 6 arises from the one for family 2 a by multiplication on the right by the $\kappa$-binomial matrix $B_{\kappa}[$ cf. (2.13)]:

$$
\boldsymbol{T}\left(\kappa\left(\alpha^{\prime}+\beta^{\prime}\right), \kappa \beta^{\prime}, \kappa \gamma^{\prime}, \alpha^{\prime}, \beta^{\prime}, \gamma^{\prime}\right)=\boldsymbol{T}\left(\alpha,-\alpha,-\alpha, \alpha^{\prime}, \beta^{\prime}, \gamma^{\prime}\right) B_{\kappa} .
$$

Indeed, this follows immediately by comparing (3.38) with (3.61).

Remark. By applying Corollary A.15 to family 2a and using (3.63), we see that family 6 also satisfies the recurrence

$$
\begin{aligned}
T(n, k)=[ & \left.\left(\alpha+\kappa \alpha^{\prime}\right) n+\left(-\alpha+2 \kappa \beta^{\prime}\right) k-\alpha+\kappa\left(\beta^{\prime}+\gamma^{\prime}\right)\right] T(n-1, k) \\
& +\left(\alpha^{\prime} n+\beta^{\prime} k+\gamma^{\prime}\right) T(n-1, k-1) \\
& +\kappa\left(-\alpha+\kappa \beta^{\prime}\right)(k+1) T(n-1, k+1)
\end{aligned}
$$

for $n \geqslant 1$. (This is a recurrence of GKPZ form: see Section 6.3 below.) It is far from obvious (at least to us) that the solution of this recurrence is independent of $\alpha$ or depends 
on $\alpha^{\prime}, \beta^{\prime}$ only via their sum, much less that it satisfies the GKP recurrence (1.1) for family 6 . But it does.

As previously mentioned, family 6 is self-dual. The action of duality on family 6 can be seen most clearly by employing the parameter $\delta^{\prime} \stackrel{\text { def }}{=} \alpha^{\prime}+\beta^{\prime}$ in place of $\alpha^{\prime}$, thereby parametrizing family 6 by the quadruplet $\left(\delta^{\prime}, \beta^{\prime}, \gamma^{\prime}, \kappa\right)$. Then duality $(2.5)$ acts by taking $\left(\delta^{\prime}, \beta^{\prime}, \gamma^{\prime}, \kappa\right) \mapsto\left(\kappa \delta^{\prime},-\kappa \beta^{\prime}, \kappa \gamma^{\prime}, 1 / \kappa\right)$.

\section{Particular cases}

- The generalized $(s,-s)$-Eulerian numbers $\left\langle\begin{array}{l}n \\ k\end{array}\right\rangle_{(s,-s)}$ [3] correspond to $\boldsymbol{\mu}=(0,1, s$, $1,-1,-s)$. They are trivial rescalings of the binomial coefficients:

$$
\left\langle\begin{array}{l}
n \\
k
\end{array}\right\rangle_{(s,-s)}=(-1)^{k} s^{n}\left(\begin{array}{l}
n \\
k
\end{array}\right)
$$

- The numbers $T(n, k)=n !\left(\begin{array}{l}n \\ k\end{array}\right)[41, \mathrm{~A} 196347]$ can be obtained from $\boldsymbol{\mu}=(1,0,0,1,0,0)$. This can be seen by applying Corollary 2.2(c) with $\kappa=1, \lambda=0$ to the binomial coefficients.

\section{Preliminaries for T-fractions and J-fractions}

In this section we review some transformation formulae for continued fractions that will be needed for our discussion in Section 5 of T-fractions and J-fractions for the GKP recurrence. The plan of this section is as follows: First we review the formula for the contraction of S-fractions or special T-fractions to J-fractions (Section 4.1). Then we review the theory of the binomial transform and its action on S-fractions, special Tfractions, and J-fractions (Section 4.2).

\subsection{Contraction}

It is a classical result, going back to the middle of the nineteenth century, that any S-fraction (1.7) can be re-expressed as a J-fraction (1.10). This operation, which is known as contraction, is given as follows:

Proposition 4.1 (Even contraction for S-fractions). We have

$$
\frac{1}{1-\frac{c_{1} t}{1-\frac{c_{2} t}{1-\frac{c_{3} t}{1-\cdots}}}}=\frac{1}{1-c_{1} t-\frac{c_{1} c_{2} t^{2}}{1-\left(c_{2}+c_{3}\right) t-\frac{c_{3} c_{4} t^{2}}{1-\left(c_{4}+c_{5}\right) t-\frac{c_{5} c_{6} t^{2}}{1-\cdots}}}},
$$


so that the J-fraction on the right-hand side has coefficients

$$
\begin{aligned}
& e_{0}=c_{1} \\
& e_{n}=c_{2 n}+c_{2 n+1} \quad \text { for } n \geqslant 1 \\
& f_{n}=c_{2 n-1} c_{2 n}
\end{aligned}
$$

The classic algebraic proof of this result, using the convergents of the continued fraction, can be found in the book of Wall [63, p. 21]. A very simple and elegant algebraic proof can be found in [18, Lemmas 1 and 2] [17, proof of Lemma 1] [12, Lemma 4.5]. A beautiful and enlightening combinatorial proof, using Flajolet's [27] representation of S-fractions (resp. J-fractions) in terms of Dyck (resp. Motzkin) paths, can be found in the lectures of Viennot [61, pp. V-31-V-32].

There is also [49] a generalization of Proposition 4.1 to a subclass of T-fractions, namely, those with $d_{i}=0$ for all even levels $i$ :

Proposition 4.2 (Even contraction for T-fractions with $\boldsymbol{d}_{\boldsymbol{i}}=\mathbf{0}$ at all even levels $\boldsymbol{i}$ ). We have

$$
\begin{aligned}
& \frac{1}{1-d_{1} t-\frac{c_{1} t}{1-\frac{c_{2} t}{1-d_{3} t-\frac{c_{3} t}{1-\cdots}}}}= \\
& \frac{1}{1-\left(c_{1}+d_{1}\right) t-\frac{c_{1} c_{2} t^{2}}{1-\left(c_{2}+c_{3}+d_{3}\right) t-\frac{c_{3} c_{4} t^{2}}{1-\left(c_{4}+c_{5}+d_{5}\right) t-\frac{c_{5} c_{6} t^{2}}{1-\cdots}}}},
\end{aligned}
$$

so that the J-fraction on the right-hand side has coefficients

$$
\begin{aligned}
& e_{0}=c_{1}+d_{1} \\
& e_{n}=c_{2 n}+c_{2 n+1}+d_{2 n+1} \quad \text { for } n \geqslant 1 \\
& f_{n}=c_{2 n-1} c_{2 n}
\end{aligned}
$$

Proposition 4.2 can be proven [49] either algebraically by manipulating the continued fraction, or combinatorially by using the representation of T-fractions (resp. J-fractions) in terms of Schröder (resp. Motzkin) paths.

\subsection{Binomial transform}

Let $\boldsymbol{a}=\left(a_{n}\right)_{n \geqslant 0}$ be a sequence with values in a commutative ring (with identity element) $R$, and let $\xi$ be an element of $R$ (or an indeterminate). Then the $\xi$-binomial 
transform of $\boldsymbol{a}$ is the sequence $\boldsymbol{b}=\left(b_{n}\right)_{n \geqslant 0}$ defined by

$$
b_{n}=\sum_{k=0}^{n}\left(\begin{array}{l}
n \\
k
\end{array}\right) a_{k} \xi^{n-k},
$$

or in other words $\boldsymbol{b}=B_{\xi} \boldsymbol{a}$ where $B_{\xi}$ is the (unit-lower-triangular) $\xi$-binomial matrix [cf., $(2.13)]$

$$
\left(B_{\xi}\right)_{n k}=\left(\begin{array}{l}
n \\
k
\end{array}\right) \xi^{n-k}
$$

If the ring $R$ contains the rationals, then we can form the exponential generating functions

$$
A(t) \stackrel{\text { def }}{=} \sum_{n=0}^{\infty} a_{n} \frac{t^{n}}{n !}, \quad B(t) \stackrel{\text { def }}{=} \sum_{n=0}^{\infty} b_{n} \frac{t^{n}}{n !}
$$

(considered as formal power series in the indeterminate $t$ ), and an easy computation shows that they are related by

$$
B(t)=e^{\xi t} A(t) .
$$

Therefore, an egf exhibiting a prefactor $e^{\xi t}$ is the necessary and sufficient signal of a $\xi$-binomial transform.

On the other hand, if we look at the ordinary generating functions

$$
a(t) \stackrel{\text { def }}{=} \sum_{n=0}^{\infty} a_{n} t^{n}, \quad b(t) \stackrel{\text { def }}{=} \sum_{n=0}^{\infty} b_{n} t^{n}
$$

(again considered as formal power series in the indeterminate $t$ ), Euler [21] showed that ${ }^{10}$

$$
b(t)=\frac{1}{1-\xi t} a\left(\frac{t}{1-\xi t}\right) .
$$

Furthermore, it turns out that whenever $a(t)$ can be expressed as an S-fraction (resp. J-fraction), then $b(t)$ can be expressed as a T-fraction (resp. J-fraction), as we shall now show.

Let us start with the case of an S-fraction. Let $\boldsymbol{c}=\left(c_{i}\right)_{i \geqslant 1}$ be indeterminates, and define the Stieltjes-Rogers polynomials $S_{n}(\boldsymbol{c}) \in \mathbb{Z}[\boldsymbol{c}]$ by

$$
\sum_{n=0}^{\infty} S_{n}\left(c_{1}, \ldots, c_{n}\right) t^{n}=\frac{1}{1-\frac{c_{1} t}{1-\frac{c_{2} t}{1-\cdots}}}
$$

\footnotetext{
${ }^{10}$ The identity (4.10) is a special case of the fundamental theorem of Riordan arrays (FTRA) [5, pp. 137144].
} 
Similarly, let $\boldsymbol{c}=\left(c_{i}\right)_{i \geqslant 1}$ and $\boldsymbol{d}=\left(d_{i}\right)_{i \geqslant 1}$ be indeterminates, and define the Thron-Rogers polynomials $T_{n}(\boldsymbol{c}, \boldsymbol{d}) \in \mathbb{Z}[\boldsymbol{c}, \boldsymbol{d}]$ by

$$
\sum_{n=0}^{\infty} T_{n}\left(c_{1}, \ldots, c_{n}, d_{1}, \ldots, d_{n}\right) t^{n}=\frac{1}{1-d_{1} t-\frac{c_{1} t}{1-d_{2} t-\frac{c_{2} t}{1-\cdots}}} .
$$

Barry [4, Proposition 3] proved the following:

Proposition 4.3 ( $\boldsymbol{\xi}$-binomial transform of S-fraction as T-fraction). We have

$$
\sum_{k=0}^{n}\left(\begin{array}{l}
n \\
k
\end{array}\right) S_{k}(\boldsymbol{c}) \xi^{n-k}=T_{n}\left(\boldsymbol{c}, \xi \mathbf{1}_{\text {odd }}\right)
$$

as an identity in $\mathbb{Z}[\boldsymbol{c}, \xi]$, where $\mathbf{1}_{\text {odd }}=(1,0,1,0, \ldots)$.

So the $\xi$-binomial transform of an S-fraction with coefficients $\boldsymbol{c}=\left(c_{i}\right)_{i \geqslant 1}$ is a T-fraction with the same coefficients $\boldsymbol{c}$ and in which $d_{i}=\xi$ (resp. 0) for odd (resp. even) $i$.

In fact, this result can be generalized [49] from S-fractions to a subclass of T-fractions, namely, those in which $d_{i}=0$ at all even levels $i$ :

Proposition 4.4 ( $\boldsymbol{\xi}$-binomial transform of a subclass of T-fractions). We have

$$
\sum_{k=0}^{n}\left(\begin{array}{l}
n \\
k
\end{array}\right) T_{k}\left(\boldsymbol{c}, \boldsymbol{d}_{\text {odd }}\right) \xi^{n-k}=T_{n}\left(\boldsymbol{c}, \boldsymbol{d}_{\text {odd }}+\xi \mathbf{1}_{\text {odd }}\right)
$$

as an identity in $\mathbb{Z}\left[\boldsymbol{c}, \boldsymbol{d}_{\mathrm{odd}}, \xi\right]$, where $\boldsymbol{d}_{\mathrm{odd}}=\left(d_{1}, 0, d_{3}, 0, \ldots\right)$ and $\mathbf{1}_{\mathrm{odd}}=(1,0,1,0, \ldots)$.

Finally, let us consider the case in which $a(t)$ can be expressed as a J-fraction. Let $\boldsymbol{e}=\left(e_{i}\right)_{i \geqslant 0}$ and $\boldsymbol{f}=\left(f_{i}\right)_{i \geqslant 1}$ be indeterminates, and define the Jacobi-Rogers polynomials $J_{n}(\boldsymbol{e}, \boldsymbol{f}) \in \mathbb{Z}[\boldsymbol{e}, \boldsymbol{f}]$ by

$$
\sum_{n=0}^{\infty} J_{n}(\boldsymbol{e}, \boldsymbol{f}) t^{n}=\frac{1}{1-e_{0} t-\frac{f_{1} t^{2}}{1-e_{1} t-\frac{f_{2} t^{2}}{1-\cdots}}} .
$$

We then have:

Proposition 4.5 ( $\boldsymbol{\xi}$-binomial transform of J-fraction). We have

$$
\sum_{k=0}^{n}\left(\begin{array}{l}
n \\
k
\end{array}\right) J_{k}(\boldsymbol{e}, \boldsymbol{f}) \xi^{n-k}=J_{n}(\boldsymbol{e}+\xi \mathbf{1}, \boldsymbol{f})
$$

as an identity in $\mathbb{Z}[\boldsymbol{e}, \boldsymbol{f}, \xi]$, where $\mathbf{1}=(1,1,1,1, \ldots)$. 
In other words, the $\xi$-binomial transform simply adds a constant $\xi$ to all the coefficients $e_{i}$. This result can be found in Aigner [1, eq. (6.15)] and Barry [4, Proposition 4], but it may well go back to the late nineteenth century.

Let us remark that all three Propositions can be proven either algebraically by manipulating the continued fraction using Euler's formula (4.10), or combinatorially by using the expressions for the Stieltjes-Rogers, Jacobi-Rogers and Thron-Rogers polynomials in terms of Dyck, Motzkin and Schröder paths, respectively. See [49] for details.

By combining the contraction formula (Proposition 4.1) with Proposition 4.5, we can alternatively write the $\xi$-binomial transform of an S-fraction as a J-fraction:

Corollary 4.6 ( $\boldsymbol{\xi}$-binomial transform of S-fraction as J-fraction). We have

$$
\sum_{k=0}^{n}\left(\begin{array}{l}
n \\
k
\end{array}\right) S_{k}(\boldsymbol{c}) \xi^{n-k}=J_{n}(\boldsymbol{e}, \boldsymbol{f})
$$

where

$$
\begin{aligned}
& e_{0}=c_{1}+\xi \\
& e_{n}=c_{2 n}+c_{2 n+1}+\xi \quad \text { for } n \geqslant 1 \\
& f_{n}=c_{2 n-1} c_{2 n}
\end{aligned}
$$

So the $\xi$-binomial transform of an S-fraction can be written either as a T-fraction (Proposition 4.3) or as a J-fraction (Corollary 4.6). Of course, the T-fraction and the J-fraction are equivalent by virtue of Proposition 4.2; otherwise put, the $\xi$-binomial transform commutes with contraction (as it must).

Similarly, by combining the contraction formula for special T-fractions (Proposition 4.2) with Proposition 4.5, we can write the $\xi$-binomial transform of a special T-fraction as a J-fraction:

Corollary 4.7 ( $\boldsymbol{\xi}$-binomial transform of special T-fraction as J-fraction). We have

$$
\sum_{k=0}^{n}\left(\begin{array}{l}
n \\
k
\end{array}\right) T_{n}\left(\boldsymbol{c}, \boldsymbol{d}_{\text {odd }}\right) \xi^{n-k}=J_{n}(\boldsymbol{e}, \boldsymbol{f})
$$

where $\boldsymbol{d}_{\mathrm{odd}}=\left(d_{1}, 0, d_{3}, 0, \ldots\right)$ and

$$
\begin{aligned}
& e_{0}=c_{1}+d_{1}+\xi \\
& e_{n}=c_{2 n}+c_{2 n+1}+d_{2 n+1}+\xi \quad \text { for } n \geqslant 1 \\
& f_{n}=c_{2 n-1} c_{2 n}
\end{aligned}
$$

Once again, the $\xi$-binomial transform commutes with contraction. 


\section{Some GKP recurrences with a T-fraction or J-fraction repre- sentation}

All of the S-fractions found in Propositions 3.6-3.11 trivially give rise to T-fractions by setting all $d_{i}=0$ and to J-fractions by contraction (Proposition 4.1). In this section we will show some less trivial cases of the GKP recurrence in which the ogf has a representation as a T-fraction or a J-fraction. However, unlike what we did for S-fractions, we shall not attempt a complete determination. One obstacle to a complete analysis is that Tfractions are highly underdetermined: corresponding to the $n$ inputs $a_{1}, \ldots, a_{n}$ there are $2 n$ parameters $c_{1}, \ldots, c_{n}$ and $d_{1}, \ldots, d_{n}$. For this reason, a systematic search for Tfractions, such as we did Section 3 for the S-fractions, does not seem at present to be feasible. A systematic search seems more feasible for the J-fractions, since they, like the S-fractions, are fully determined (modulo degenerate cases): the $2 n$ inputs $a_{1}, \ldots, a_{2 n}$ give rise to $2 n$ parameters $e_{0}, \ldots, e_{n-1}$ and $f_{1}, \ldots, f_{n}$. But this search is more involved than what we have done for S-fractions, and we have not yet carried it to completion. So the examples given in this section are surely only the tip of the iceberg.

We begin by presenting some examples of GKP recurrences whose ogf can be expressed as a T-fraction or J-fraction arising from a binomial transform (Section 5.1). We then present some examples whose ogf can be expressed as a T-fraction not arising from a binomial transform (Section 5.2) or a J-fraction not arising from a binomial transform (Section 5.3).

\subsection{Examples of GKP recurrences with T-fraction or J-fraction arising from the binomial transform}

We will now use the $\xi$-binomial transform to find some cases of the GKP recurrence (1.1) in which the ogf has a representation as a T-fraction or J-fraction. More specifically, the row-generating polynomials $P_{n}(x)$ of our families $7 \mathrm{a}$ and $7 \mathrm{~b}$ will be the $\xi$-binomial transforms, for suitable $\xi$, of the row-generating polynomials for families $3 \mathrm{a}$ and $3 \mathrm{~b}$ : that is, we will have

$$
P_{n}\left(x ; \boldsymbol{\mu}^{\prime}\right)=\sum_{k=0}^{n}\left(\begin{array}{l}
n \\
k
\end{array}\right) P_{k}(x ; \boldsymbol{\mu}) \xi^{n-k}
$$

for suitable $\boldsymbol{\mu}, \boldsymbol{\mu}^{\prime}$ and $\xi$. The T-fractions for families $7 \mathrm{a}$ and $7 \mathrm{~b}$ will then be deduced from the S-fractions for families $3 \mathrm{a}$ and $3 \mathrm{~b}$ (Proposition 3.8) by using Proposition 4.3, and the J-fractions will be found in a similar way by using Corollary 4.6.

Let us observe that, under duality, the coefficients $\boldsymbol{c}$ and $\boldsymbol{d}$ in the T-fraction behave in the same way as the coefficient $\boldsymbol{c}$ of the S-fraction: namely, $c_{i}(x) \rightarrow x c_{i}(1 / x)$ and $d_{i}(x) \rightarrow x d_{i}(1 / x)$. Therefore, if $f(x, t ; \boldsymbol{\mu})$ has a T-fraction representation with coefficients that are affine in $x$, then $f(x, t ; D \boldsymbol{\mu})$ has also a T-fraction representation with coefficients that are also affine in $x$ : namely, $a+b x \rightarrow b+a x$.

\subsubsection{Families $7 \mathrm{a}$ and $7 \mathrm{~b}$}

Families $7 \mathrm{a}$ and $7 \mathrm{~b}$ are defined as follows: 
F7a. $\boldsymbol{\mu}=\left(0, \beta, \gamma, 0, \beta^{\prime}, \gamma^{\prime}\right)$

F7b. $\boldsymbol{\mu}=\left(\alpha,-\alpha, \gamma, \alpha^{\prime},-\alpha^{\prime}, \gamma^{\prime}\right)$

Please note that family $7 \mathrm{a}$ is a generalization of family $3 \mathrm{a}$ (which corresponds to $\gamma=0$ ), while family $7 \mathrm{~b}$ is a generalization of family $3 \mathrm{~b}$ (which corresponds to $\gamma^{\prime}=0$ ). Furthermore, family $7 \mathrm{a}$ is also a generalization of family $4 \mathrm{a}$ (which corresponds to $\gamma / \beta=1+\gamma^{\prime} / \beta^{\prime}$ ), while family $7 \mathrm{~b}$ is a generalization of family $4 \mathrm{~b}$ (which corresponds to $\gamma^{\prime} / \alpha^{\prime}=1+\gamma / \alpha$ ). Family 7 a corresponds to the case of the GKP recurrence (1.1) in which the coefficients depend only on $k$, while family $7 \mathrm{~b}$ is the dual case in which the coefficients depend only on $n-k$.

The key fact is the following:

Proposition 5.1 (Families $7 \mathrm{a}$ and $7 \mathrm{~b}$ as binomial transforms). The row-generating polynomials of family 7 a are the $\gamma$-binomial transform of those of family $3 a$ :

$$
P_{n}\left(x ; 0, \beta, \gamma, 0, \beta^{\prime}, \gamma^{\prime}\right)=\sum_{k=0}^{n}\left(\begin{array}{l}
n \\
k
\end{array}\right) P_{n}\left(x ; 0, \beta, 0,0, \beta^{\prime}, \gamma^{\prime}\right) \gamma^{n-k} .
$$

Similarly, the row-generating polynomials of family $7 \mathrm{~b}$ are the $\left(\gamma^{\prime} x\right)$-binomial transform of those of family $3 b$ :

$$
P_{n}\left(x ; \alpha,-\alpha, \gamma, \alpha^{\prime},-\alpha^{\prime}, \gamma^{\prime}\right)=\sum_{k=0}^{n}\left(\begin{array}{l}
n \\
k
\end{array}\right) P_{n}\left(x ; \alpha,-\alpha, \gamma, \alpha^{\prime},-\alpha^{\prime}, 0\right)\left(\gamma^{\prime} x\right)^{n-k} .
$$

Proof. Family 7a is Neuwirth's [40, Theorem 18] case $\alpha^{\prime}=0$ specialized to $\alpha=0$. Its egf is given by $[2$, eq. (A8)]:

$$
F(x, t)=e^{\gamma t}\left[1+\frac{\beta^{\prime} x}{\beta}\left(1-e^{\beta t}\right)\right]^{-\left(\beta^{\prime}+\gamma^{\prime}\right) / \beta^{\prime}} .
$$

Comparing this with (3.47), we see that family $7 \mathrm{a}$ is the $\gamma$-binomial transform of family $3 \mathrm{a}$. (We will give an alternate proof of this result in Appendix A: see the Remark after Corollary A.6.)

Similarly, family 7b is Spivey's case (S1) $\beta=-\alpha$ specialized to $\beta^{\prime}=-\alpha^{\prime}$ [52]. Its egf is given by $[2$, eq. (A4)]:

$$
F(x, t)=e^{\gamma^{\prime} x t}\left[1+\frac{\alpha}{\alpha^{\prime} x}\left(1-e^{\alpha^{\prime} x t}\right)\right]^{-(\alpha+\gamma) / \alpha} .
$$

Comparing this with (3.48), we see that family $7 \mathrm{~b}$ is the $\left(\gamma^{\prime} x\right)$-binomial transform of family $3 \mathrm{~b}$.

Remark. Since $B_{\xi_{1}} B_{\xi_{2}}=B_{\xi_{1}+\xi_{2}}$, it follows that the $\xi$-binomial transform of family 7a is simply family $7 \mathrm{a}$ with $\gamma \rightarrow \gamma+\xi$, and the $(\xi x)$-binomial transform of family $7 \mathrm{~b}$ is family $7 \mathrm{~b}$ with $\gamma^{\prime} \rightarrow \gamma^{\prime}+\xi$.

Combining this with Propositions 3.8 and 4.3, we obtain: 
Corollary 5.2 (T-fraction for families $7 \mathrm{a}$ and $7 \mathrm{~b})$. The ogf $f(x, t ; \boldsymbol{\mu})$ for the recurrence (1.1) with $\boldsymbol{\mu}=\left(0, \beta, \gamma, 0, \beta^{\prime}, \gamma^{\prime}\right)$ has a T-type continued fraction representation in the ring $\mathbb{Z}\left[x ; \beta, \gamma, \beta^{\prime}, \gamma^{\prime}\right][[t]]$ with coefficients

$$
\begin{aligned}
c_{2 k-1} & =\left(\gamma^{\prime}+k \beta^{\prime}\right) x, & c_{2 k} & =k\left(\beta+\beta^{\prime} x\right), \\
d_{2 k-1} & =\gamma, & d_{2 k} & =0
\end{aligned}
$$

Moreover, an analogous $T$-type representation exists in $\mathbb{Z}\left[x ; \alpha, \gamma, \alpha^{\prime}, \gamma^{\prime}\right][[t]]$ for the dual parameter $\boldsymbol{\mu}=\left(\alpha,-\alpha, \gamma, \alpha^{\prime},-\alpha^{\prime}, \gamma^{\prime}\right)$ with coefficients

$$
\begin{aligned}
c_{2 k-1} & =\gamma+k \alpha, & & c_{2 k}=k\left(\alpha+\alpha^{\prime} x\right), \\
d_{2 k-1} & =\gamma^{\prime} x, & d_{2 k} & =0
\end{aligned}
$$

Similarly, combining Proposition 5.1 with Proposition 3.8 and Corollary 4.6, we obtain:

Corollary 5.3 (J-fraction for families $7 \mathrm{a}$ and $7 \mathrm{~b})$. The ogf $f(x, t ; \boldsymbol{\mu})$ for the recurrence (1.1) with $\boldsymbol{\mu}=\left(0, \beta, \gamma, 0, \beta^{\prime}, \gamma^{\prime}\right)$ has a J-type continued fraction representation in the ring $\mathbb{Z}\left[x ; \beta, \gamma, \beta^{\prime}, \gamma^{\prime}\right][[t]]$ with coefficients

$$
\begin{aligned}
& e_{n}=\left[\gamma+\left(\beta^{\prime}+\gamma^{\prime}\right) x\right]+n\left(\beta+2 \beta^{\prime} x\right) \\
& f_{n}=n\left(\gamma^{\prime}+n \beta^{\prime}\right) x\left(\beta+\beta^{\prime} x\right)
\end{aligned}
$$

Moreover, an analogous J-type representation exists in $\mathbb{Z}\left[x ; \alpha, \gamma, \alpha^{\prime}, \gamma^{\prime}\right][[t]]$ for the dual parameter $\boldsymbol{\mu}=\left(\alpha,-\alpha, \gamma, \alpha^{\prime},-\alpha^{\prime}, \gamma^{\prime}\right)$ with coefficients

$$
\begin{aligned}
e_{n} & =\left(\alpha+\gamma+\gamma^{\prime} x\right)+n\left(2 \alpha+\alpha^{\prime} x\right) \\
f_{n} & =n(\gamma+n \alpha)\left(\alpha+\alpha^{\prime} x\right)
\end{aligned}
$$

Remarks. 1. The dual of $\boldsymbol{\mu}=\left(0, \beta, \gamma, 0, \beta^{\prime}, \gamma^{\prime}\right)$ is $D \boldsymbol{\mu}=\left(\beta^{\prime},-\beta^{\prime}, \gamma^{\prime} ; \beta,-\beta, \gamma\right)$, so family $7 \mathrm{~b}$ is obtained from family $7 \mathrm{a}$ by applying duality followed by the map $\left(\beta^{\prime}, \gamma^{\prime}, \beta, \gamma\right) \mapsto$ $\left(\alpha, \gamma, \alpha^{\prime}, \gamma^{\prime}\right)$. The T-fraction for family $7 \mathrm{~b}$ can then be deduced by duality from the one for family $7 \mathrm{a}$, by using the observations made at the beginning of this section.

2. The special case $\boldsymbol{\mu}=(0,0,1 ; 0,1,0)$ of family 7 a leads to the injective numbers $\operatorname{Inj}(n, k)=n ! /(n-k) ![26][41, \mathrm{~A} 008279]$. The dual array $T(n, k)=n ! / k ![41, \mathrm{~A} 094587]$ is the special case $\boldsymbol{\mu}=(1,-1,0 ; 0,0,1)$ of family $7 \mathrm{~b}$.

3. As noted earlier, families $2 \mathrm{a}$ and $2 \mathrm{~b}$ are equivalent, at the level of the matrices $\boldsymbol{T}(\boldsymbol{\mu})$, to special cases of families $3 \mathrm{a}$ and $3 \mathrm{~b}$, respectively. Therefore, the $\gamma$-binomial transform of family $2 \mathrm{a}$ is a special case of family $7 \mathrm{a}$, and the $\left(\gamma^{\prime} x\right)$-binomial transform of family $2 \mathrm{~b}$ is a special case of family $7 \mathrm{~b}$. 


\subsection{Examples of GKP recurrences with T-fraction not arising from the bi- nomial transform}

\subsubsection{Families 8a and 8b: Generalized Ward polynomials and their dual}

In a very recent paper [19], Elvey Price and Sokal have given [19, Theorem 1.2] a T-fraction for the ordinary generating function of some polynomials $W_{n}(x, u, z, w)$ that generalize the Ward polynomials [41, A134991/A181996/A269939]: the coefficients of this T-fraction are

$$
c_{i}=x+(i-1) u, \quad d_{i}=z+(i-1) w .
$$

Moreover, in the special case $u=x$, these polynomials satisfy a linear recurrence of GKP form [19, Corollary B.2]: setting $W_{n}(x, x, z, w)=\sum_{k=0}^{n} W_{n, k}(z, w) x^{k}$, the triangular array $\left(W_{n, k}(z, w)\right)_{0 \leqslant k \leqslant n}$ satisfies

$$
W_{n, k}=(w k+z) W_{n-1, k}+(n+k-1) W_{n-1, k-1} \quad \text { for } n \geqslant 1
$$

with initial condition $W_{0, k}=\delta_{k 0}$. So the ogf of the GKP recurrence with $\boldsymbol{\mu}=(0, \beta, \gamma, 1,1$, $-1)$ has a T-fraction with coefficients $c_{i}=i x, d_{i}=\gamma+(i-1) \beta$. And by applying the scaling $S_{1, \lambda}$ [cf. (2.2)] to this T-fraction, we deduce that the ogf of the GKP recurrence with $\boldsymbol{\mu}=$ $\left(0, \beta, \gamma, \alpha^{\prime}, \alpha^{\prime},-\alpha^{\prime}\right)$ has a T-fraction with coefficients $c_{i}=i \alpha^{\prime} x, d_{i}=\gamma+(i-1) \beta$. Finally, by applying duality to this result, we deduce that the ogf of the GKP recurrence with $\boldsymbol{\mu}=\left(2 \widehat{\alpha},-\widehat{\alpha},-\widehat{\alpha}, \alpha^{\prime},-\alpha^{\prime}, \gamma^{\prime}\right)$ has a T-fraction with coefficients $c_{i}=i \widehat{\alpha}, d_{i}=\gamma^{\prime}+(i-1) \alpha^{\prime}$. (We use the change of parameters $\alpha \stackrel{\text { def }}{=} 2 \widehat{\alpha}$ to avoid fractions.)

We summarize this by defining family 8 a (the rescaled generalized Ward polynomials at $u=x$ ) and family $8 \mathrm{~b}$ (their dual):

F8a. $\boldsymbol{\mu}=\left(0, \beta, \gamma, \alpha^{\prime}, \alpha^{\prime},-\alpha^{\prime}\right)$

F8b. $\boldsymbol{\mu}=\left(2 \widehat{\alpha},-\widehat{\alpha},-\widehat{\alpha}, \alpha^{\prime},-\alpha^{\prime}, \gamma^{\prime}\right)$

We then have:

Proposition 5.4 (T-fraction for families $8 \mathrm{a}$ and $8 \mathrm{~b})$. The ogf $f(x, t ; \boldsymbol{\mu})$ for the recurrence (1.1) with $\boldsymbol{\mu}=\left(0, \beta, \gamma, \alpha^{\prime}, \alpha^{\prime},-\alpha^{\prime}\right)$ has a T-type continued fraction representation in the ring $\mathbb{Z}\left[x ; \beta, \gamma, \alpha^{\prime}\right][[t]]$ with coefficients

$$
c_{i}=i \alpha^{\prime} x, \quad d_{i}=\gamma+(i-1) \beta .
$$

Moreover, an analogous $T$-type representation exists in $\mathbb{Z}\left[x ; \widehat{\alpha}, \alpha^{\prime}, \gamma^{\prime}\right][[t]]$ for the dual parameter $\boldsymbol{\mu}=\left(2 \widehat{\alpha},-\widehat{\alpha},-\widehat{\alpha}, \alpha^{\prime},-\alpha^{\prime}, \gamma^{\prime}\right)$ with coefficients

$$
c_{i}=i \widehat{\alpha}, \quad d_{i}=\left[\gamma^{\prime}+(i-1) \alpha^{\prime}\right] x .
$$

Let us remark that the proof of this result in [19] is rather indirect. The polynomials $W_{n}(x, u, z, w)$ are defined combinatorially, as generating polynomials for "superaugmented perfect matchings" of $[2 n]$ with suitable weights. The T-fraction (5.10) and the recurrence (5.11) are then proven by combinatorial arguments (of very different forms). It is an interesting open problem to prove Proposition 5.4 by direct arguments leading from the recurrence to the T-fraction (or vice versa). 


\subsubsection{Families 9a and 9b: Conjectured T- and J-fractions}

We have found empirically a T-fraction for the family

F9a. $\boldsymbol{\mu}=\left(0, \beta,(\kappa+1) \beta,-\widehat{\alpha}^{\prime}, 2 \widehat{\alpha}^{\prime}, \kappa \widehat{\alpha}^{\prime}\right)$

and its dual

F9b. $\boldsymbol{\mu}=\left(\alpha,-2 \alpha, \kappa \alpha, \alpha^{\prime},-\alpha^{\prime},(\kappa+1) \alpha^{\prime}\right)$

(In family 9a we have introduced the change of parameters $\alpha^{\prime} \stackrel{\text { def }}{=}-\widehat{\alpha}^{\prime}$ in order to have plus signs in the T-fraction.)

Conjecture 5.5 (T-fraction for families $9 \mathrm{a}$ and $9 \mathrm{~b})$. The ogf $f(x, t ; \boldsymbol{\mu})$ for the recurrence (1.1) with $\boldsymbol{\mu}=\left(0, \beta,(\kappa+1) \beta,-\widehat{\alpha}^{\prime}, 2 \widehat{\alpha}^{\prime}, \kappa \widehat{\alpha}^{\prime}\right)$ has a T-type continued fraction representation in the ring $\mathbb{Z}\left[x ; \beta, \widehat{\alpha}^{\prime}, \kappa\right][[t]]$ with coefficients

$$
\begin{aligned}
& c_{2 k-1}=(\kappa+k) \widehat{\alpha}^{\prime} x, \quad c_{2 k}=k \widehat{\alpha}^{\prime} x, \\
& d_{2 k-1}=(\kappa+2 k-1) \beta, \quad d_{2 k}=0
\end{aligned}
$$

Moreover, an analogous T-type representation exists in $\mathbb{Z}\left[x ; \alpha, \alpha^{\prime}, \kappa\right][[t]]$ for the dual parameter $\boldsymbol{\mu}=\left(\alpha,-2 \alpha, \kappa \alpha, \alpha^{\prime},-\alpha^{\prime},(\kappa+1) \alpha^{\prime}\right)$ with coefficients

$$
\begin{array}{ll}
c_{2 k-1}=(\kappa+k) \alpha, & c_{2 k}=k \alpha, \\
d_{2 k-1}=(\kappa+2 k-1) \alpha^{\prime} x, & d_{2 k}=0
\end{array}
$$

We have verified Conjecture 5.5 for $0 \leqslant n \leqslant 20$.

It will be observed that the T-fractions of Conjecture 5.5 have $d_{i}=0$ at all even levels $i$. Therefore, Proposition 4.2 implies that Conjecture 5.5 is equivalent to:

Conjecture 5.6 (J-fraction for families $9 \mathrm{a}$ and $9 \mathrm{~b})$. The ogf $f(x, t ; \boldsymbol{\mu})$ for the recurrence (1.1) with $\boldsymbol{\mu}=\left(0, \beta,(\kappa+1) \beta, \alpha^{\prime},-2 \alpha^{\prime},-\kappa \alpha^{\prime}\right)$ has a J-type continued fraction representation in the ring $\mathbb{Z}\left[x ; \beta, \widehat{\alpha}^{\prime}, \kappa\right][[t]]$ with coefficients

$$
\begin{aligned}
& e_{n}=(\kappa+2 n+1)\left(\beta+\widehat{\alpha}^{\prime} x\right) \\
& f_{n}=n(\kappa+n)\left(\widehat{\alpha}^{\prime} x\right)^{2}
\end{aligned}
$$

Moreover, an analogous J-type representation exists in $\mathbb{Z}\left[x ; \alpha, \alpha^{\prime}, \kappa\right][[t]]$ for the dual parameter $\boldsymbol{\mu}=\left(\alpha,-2 \alpha, \kappa \alpha, \alpha^{\prime},-\alpha^{\prime},(\kappa+1) \alpha^{\prime}\right)$ with coefficients

$$
\begin{aligned}
& e_{n}=(\kappa+2 n+1)\left(\alpha+\alpha^{\prime} x\right) \\
& f_{n}=n(\kappa+n) \alpha^{2}
\end{aligned}
$$

Remark. Since the recurrence for family 9a has a term $(-n+2 k+\kappa) \widehat{\alpha}^{\prime} T(n-1, k-1)$ on the right-hand side, and the recurrence for family $9 \mathrm{~b}$ has a term $(n-2 k) \alpha T(n-1, k)-$ neither of which is nonnegative for all $0 \leqslant k \leqslant n$ - it is far from obvious that the resulting coefficients $T(n, k)$ will be polynomials with nonnegative coefficients in $x$ and $\beta, \kappa, \widehat{\alpha}^{\prime}$ (or $x$ and $\left.\alpha, \kappa, \alpha^{\prime}\right)$. But this property is an immediate consequence of the T-fractions or J-fractions. Indeed, the T-fractions imply the much stronger property of coefficientwise Hankel-total positivity (see Section 6.2 below). It would be good to understand, directly from the recurrence, why one gets polynomials with nonnegative coefficients. 


\subsection{Examples of GKP recurrences with J-fraction not arising from the bino- mial transform}

\subsubsection{Family 1c}

Family 1c is defined as follows:

F1c. $\boldsymbol{\mu}=\left(0, \beta, \gamma, \alpha^{\prime},-\alpha^{\prime}, \gamma^{\prime}\right)$

It is a simultaneous generalization of family $1 \mathrm{a}$ (which corresponds to $\gamma=0$ ) and family $1 \mathrm{~b}$ (which corresponds to $\gamma^{\prime}=0$ ). Family 1c is self-dual: duality acts by interchanging $(\beta, \gamma) \leftrightarrow\left(\alpha^{\prime}, \gamma^{\prime}\right)$.

Family 1c is Spivey's [52] case (S3) $\alpha / \beta=\alpha^{\prime} / \beta^{\prime}+1$ specialized to $\alpha=0$. Its egf is $[2$, eq. $(\mathrm{A} 2)]$

$$
F(x, t)=e^{(\gamma / \beta)\left(\beta-\alpha^{\prime} x\right) t}\left(\frac{\beta-\alpha^{\prime} x e^{\left(\beta-\alpha^{\prime} x\right) t}}{\beta-\alpha^{\prime} x}\right)^{-\gamma / \beta-\gamma^{\prime} / \alpha^{\prime}}
$$

For family 1c we have the following J-fraction:

Proposition 5.7 (J-fraction for family 1c). The ogf $f(x, t ; \boldsymbol{\mu})$ for the recurrence (1.1) with $\boldsymbol{\mu}=\left(0, \beta, \gamma, \alpha^{\prime},-\alpha^{\prime}, \gamma^{\prime}\right)$ has a J-type continued fraction representation in the ring $\mathbb{Z}\left[x ; \beta, \gamma, \alpha^{\prime}, \gamma^{\prime}\right][[t]]$ with coefficients

$$
\begin{aligned}
& e_{n}=(\gamma+n \beta)+\left(\gamma^{\prime}+n \alpha^{\prime}\right) x \\
& f_{n}=n\left[\beta \gamma^{\prime}+\gamma \alpha^{\prime}+(n-1) \beta \alpha^{\prime}\right] x
\end{aligned}
$$

Proposition 5.7 is in fact a special case of a recent result of Zhu [68], as will be discussed in Section 6.3.

\section{Open questions}

We conclude this paper by proposing some open problems that arise naturally from our work.

\subsection{T-fractions, J-fractions, and binomial transforms}

The main result of the present paper, Theorem 3.1, is a complete list of all families of parameters $\boldsymbol{\mu} \in \mathbb{C}^{6}$ for which the ordinary generating function (1.3) of the GKP recurrence has an S-fraction expansion (1.7) with coefficients $c_{1}, c_{2}, \ldots$ that are polynomials in $x$ (rather than rational functions). In Section 5 we exhibited some additional families where the ogf (1.3) has an expansion as a T-fraction (1.9) or a J-fraction (1.10) with polynomial coefficients, but this list was not systematic. Some of these families arose from the $\xi$ binomial transform, but even this sublist was not systematic. This naturally suggests the following open problems: 
Problem 6.1 (Classification of T-fractions). Determine all parameters $\boldsymbol{\mu} \in \mathbb{C}^{6}$ for which the ordinary generating function (1.3) has a T-fraction expansion (1.9) with coefficients $c_{1}, c_{2}, \ldots$ and $d_{1}, d_{2}, \ldots$ that are polynomials in $x$.

Problem 6.2 (Classification of J-fractions). Determine all parameters $\boldsymbol{\mu} \in \mathbb{C}^{6}$ for which the ordinary generating function (1.3) has a J-fraction expansion (1.10) with coefficients $e_{0}, e_{1}, \ldots$ and $f_{1}, f_{2}, \ldots$ that are polynomials in $x$.

Problem 6.3 (Classification of $\boldsymbol{\xi}$-binomial transforms). Determine all parameter sets $\left(\boldsymbol{\mu}, \boldsymbol{\mu}^{\prime}, \xi_{0}, \xi_{1}\right) \in \mathbb{C}^{14}$ for which

$$
P_{n}\left(x ; \boldsymbol{\mu}^{\prime}\right)=\sum_{k=0}^{n}\left(\begin{array}{l}
n \\
k
\end{array}\right) P_{k}(x ; \boldsymbol{\mu})\left(\xi_{0}+\xi_{1} x\right)^{n-k} .
$$

In the special case $\xi_{1}=0$, this problem becomes: Determine all parameter sets $\left(\boldsymbol{\mu}, \boldsymbol{\mu}^{\prime}, \xi\right) \in \mathbb{C}^{13}$ for which

$$
P_{n}\left(x ; \boldsymbol{\mu}^{\prime}\right)=\sum_{k=0}^{n}\left(\begin{array}{l}
n \\
k
\end{array}\right) P_{k}(x ; \boldsymbol{\mu}) \xi^{n-k}
$$

or equivalently

$$
\boldsymbol{T}\left(\boldsymbol{\mu}^{\prime}\right)=B_{\xi} \boldsymbol{T}(\boldsymbol{\mu})
$$

where $B_{\xi}$ is the $\xi$-binomial matrix (4.6).

Remark. The special case $\xi_{0}=0$ can also be written by a formula similar to (6.3). Indeed, (6.1) reads

$$
\sum_{j=0}^{n} T\left(n, j ; \boldsymbol{\mu}^{\prime}\right) x^{j}=\sum_{k=0}^{n}\left(\begin{array}{l}
n \\
k
\end{array}\right) \sum_{j=0}^{k} T(k, j ; \boldsymbol{\mu}) x^{j}(\xi x)^{n-k} .
$$

Extracting the coefficient of $x^{\ell}$ and then substituting $\ell=n-k+m$, we obtain

$$
T\left(n, n-m ; \boldsymbol{\mu}^{\prime}\right)=\sum_{k=0}^{n}\left(\begin{array}{l}
n \\
k
\end{array}\right) \xi^{n-k} T(k, k-m ; \boldsymbol{\mu})
$$

or in other words

$$
T\left(n, m ; D \boldsymbol{\mu}^{\prime}\right)=\sum_{k=0}^{n}\left(\begin{array}{l}
n \\
k
\end{array}\right) \xi^{n-k} T(k, m ; D \boldsymbol{\mu}),
$$

i.e.

$$
\boldsymbol{T}\left(D \boldsymbol{\mu}^{\prime}\right)=B_{\xi} \boldsymbol{T}(D \boldsymbol{\mu}),
$$

which is is just (6.3) applied to the duals $D \boldsymbol{\mu}^{\prime}$ and $D \boldsymbol{\mu}$. So the two special cases $\xi_{0}=0$ and $\xi_{1}=0$ are related by duality, and we obtain an essentially new problem only when both $\xi_{0}$ and $\xi_{1}$ are nonzero.

A related problem, in which the $\xi$-binomial matrix acts on the right, is: 
Problem 6.4 (Classification of $\boldsymbol{x}$-shift transforms). Determine all parameter sets $\left(\boldsymbol{\mu}, \boldsymbol{\mu}^{\prime}\right.$, $\xi) \in \mathbb{C}^{13}$ for which

$$
P_{n}\left(x ; \boldsymbol{\mu}^{\prime}\right)=P_{n}(x+\xi ; \boldsymbol{\mu})
$$

or equivalently

$$
\boldsymbol{T}\left(\boldsymbol{\mu}^{\prime}\right)=\boldsymbol{T}(\boldsymbol{\mu}) B_{\xi} .
$$

In Section 2.1 we mentioned that a brute-force computation using $n=0,1,2,3$ showed that the only solutions to the equations $P_{n}\left(x ; \boldsymbol{\mu}^{\prime}\right)=P_{n}(x+\xi ; \boldsymbol{\mu})$ valid for generic parameters $\boldsymbol{\mu}$ are the identity map $\left(\xi=0, \boldsymbol{\mu}^{\prime}=\boldsymbol{\mu}\right)$ and the Zhu involution $\left(\xi=-\beta / \beta^{\prime}\right.$ with $\boldsymbol{\mu}^{\prime}$ given by (2.8)). However, there almost certainly do exist solutions that are valid for lower-dimensional sets of $\boldsymbol{\mu}$; the goal of Problem 6.4 is to find them all.

Finally, Problems 6.3 and 6.4 can be amalgamated as follows:

Problem 6.5 (Classification of two-sided $\boldsymbol{\xi}$-binomial transforms). Determine all parameter sets $\left(\boldsymbol{\mu}, \boldsymbol{\mu}^{\prime}, \xi_{0}, \xi_{1}, \xi_{2}\right) \in \mathbb{C}^{15}$ for which

$$
P_{n}\left(x ; \boldsymbol{\mu}^{\prime}\right)=\sum_{k=0}^{n}\left(\begin{array}{l}
n \\
k
\end{array}\right) P_{k}\left(x+\xi_{2} ; \boldsymbol{\mu}\right)\left(\xi_{0}+\xi_{1} x\right)^{n-k} .
$$

\subsection{Coefficientwise Hankel-total positivity}

We begin by recalling that a finite or infinite matrix of real numbers is called totally positive $(\mathrm{TP})$ if all its minors are nonnegative, and totally positive of order $r\left(\mathrm{TP}_{r}\right)$ if all its minors of size $\leqslant r$ are nonnegative. Background information on totally positive matrices can be found in $[25,31,35,43]$; they have application to many fields of pure and applied mathematics. In particular, it is known [32, Théorème 9] [43, section 4.6] that an infinite Hankel matrix $\left(a_{i+j}\right)_{i, j \geqslant 0}$ of real numbers is totally positive if and only if the underlying sequence $\left(a_{n}\right)_{n \geqslant 0}$ is a Stieltjes moment sequence, i.e. the moments of a positive measure on $[0, \infty)$.

Let us now consider sequences and matrices, not of real numbers, but of polynomials (with integer or real coefficients) in one or more indeterminates $\mathbf{x}$ : in applications they will often be generating polynomials that enumerate some combinatorial objects with respect to one or more statistics. We equip the polynomial ring $\mathbb{R}[\mathbf{x}]$ with the coefficientwise partial order: that is, we say that $P$ is nonnegative (and write $P \succeq 0$ ) in case $P$ is a polynomial with nonnegative coefficients. We then say that a matrix with entries in $\mathbb{R}[\mathbf{x}]$ is coefficientwise totally positive if all its minors are polynomials with nonnegative coefficients; and analogously for coefficientwise total positivity of order $r$. We say that a sequence $\boldsymbol{a}=\left(a_{n}\right)_{n \geqslant 0}$ with entries in $\mathbb{R}[\mathbf{x}]$ is coefficientwise Hankel-totally positive if its associated infinite Hankel matrix is coefficientwise totally positive; and likewise for the version of order $r$. Coefficientwise Hankel-total positivity of a sequence of polynomials $\left(P_{n}(\mathbf{x})\right)_{n \geqslant 0}$ obviously implies the pointwise Hankel-total positivity (i.e. the Stieltjes moment property) for all $\mathbf{x} \geqslant \mathbf{0}$, but it is vastly stronger.

The key fact connecting S-fractions to coefficientwise Hankel-total positivity is the following result $[48,49]$, which is an immediate consequence of old ideas of Viennot [61, pp. IV-13-IV-15]: 
Theorem 6.6 (Total positivity of S-fractions). Let $\boldsymbol{c}=\left(c_{i}\right)_{i \geqslant 1}$ be indeterminates, and define the Stieltjes-Rogers polynomials $S_{n}(\boldsymbol{c}) \in \mathbb{Z}[\boldsymbol{c}]$ by

$$
\sum_{n=0}^{\infty} S_{n}\left(c_{1}, \ldots, c_{n}\right) t^{n}=\frac{1}{1-\frac{c_{1} t}{1-\frac{c_{2} t}{1-\cdots}}}
$$

Then the sequence $\boldsymbol{S}=\left(S_{n}(\boldsymbol{c})\right)_{n \geqslant 0}$ is coefficientwise Hankel-totally positive in the indeterminates $\boldsymbol{c}$.

In particular, if we specialize the $c_{i}$ to be polynomials with nonnegative real coefficients in some indeterminates $\mathbf{x}$, then the specialized sequence $\boldsymbol{S}=\left(S_{n}(\boldsymbol{c})\right)_{n \geqslant 0}$ is coefficientwise Hankel-totally positive in the indeterminates $\mathbf{x}$.

Moreover, this result generalizes to T-fractions [48, 49]:

Theorem 6.7 (Total positivity of T-fractions). Let $\boldsymbol{c}=\left(c_{i}\right)_{i \geqslant 1}$ and $\boldsymbol{d}=\left(d_{i}\right)_{i \geqslant 1}$ be indeterminates, and define the Thron-Rogers polynomials $T_{n}(\boldsymbol{c}, \boldsymbol{d}) \in \mathbb{Z}[\boldsymbol{c}, \boldsymbol{d}]$ by

$$
\sum_{n=0}^{\infty} T_{n}\left(c_{1}, \ldots, c_{n}, d_{1}, \ldots, d_{n}\right) t^{n}=\frac{1}{1-d_{1} t-\frac{c_{1} t}{1-d_{2} t-\frac{c_{2} t}{1-\cdots}}} .
$$

Then the sequence $\boldsymbol{T}=\left(T_{n}(\boldsymbol{c}, \boldsymbol{d})\right)_{n \geqslant 0}$ is coefficientwise Hankel-totally positive in the indeterminates $\boldsymbol{c}$ and $\boldsymbol{d}$.

In particular, if we specialize the $c_{i}$ and $d_{i}$ to be polynomials with nonnegative real coefficients in some indeterminates $\mathbf{x}$, then the specialized sequence $\boldsymbol{T}=\left(T_{n}(\boldsymbol{c}, \boldsymbol{d})\right)_{n \geqslant 0}$ is coefficientwise Hankel-totally positive in the indeterminates $\mathbf{x}$.

There is also a result for J-fractions [48,49], but it is more delicate; Hankel-total positivity does not hold coefficientwise in the parameters $\boldsymbol{e}=\left(e_{i}\right)_{i \geqslant 0}$ and $\boldsymbol{f}=\left(f_{i}\right)_{i \geqslant 1}$, but only when those parameters satisfy suitable inequalities. The paper [49] describing this general theory for S-, T- and J-fractions is not yet publicly available, but the foregoing results (as well as some much stronger ones) can be found (with proofs) in [42, section 9].

Now, simple inspection of the results summarized in Propositions 3.6-3.11 shows that all the coefficients $c_{i}$ are polynomials with nonnegative integer coeffcients in the variable $x$ and the parameters $\alpha, \beta, \gamma, \alpha^{\prime}, \beta^{\prime}, \gamma^{\prime}, \kappa$ (or a subset of them). Therefore, Theorem 6.6 implies that the corresponding sequence $\boldsymbol{P}=\left(P_{n}(x ; \boldsymbol{\mu})\right)_{n \geqslant 0}$ of row-generating polynomials is coefficientwise Hankel-totally positive, jointly in all these indeterminates. Similarly, in Corollary 5.2 and Proposition 5.4, all the coefficients $c_{i}$ and $d_{i}$ are polynomials with nonnegative integer coeffcients in $x$ and the parameters, so Theorem 6.7 implies coefficientwise Hankel-total positivity. Our results in this paper therefore imply: 
Corollary 6.8. In all the families 1a-6 of Theorem 3.1, as well as families 7a and $7 b$ of Corollary 5.2 and families $8 a$ and 86 of Proposition 5.4, the sequence $\boldsymbol{P}=\left(P_{n}(x ; \boldsymbol{\mu})\right)_{n \geqslant 0}$ of row-generating polynomials is coefficientwise Hankel-totally positive, jointly in all the indeterminates $x$ and $\alpha, \beta, \gamma, \alpha^{\prime}, \beta^{\prime}, \gamma^{\prime}, \kappa$.

But vastly more appears to be true: it seems that coefficientwise Hankel-total positivity is a general property of the GKP recurrence, not just of the special families 1a-8b studied here. Namely, we conjecture:

Conjecture 6.9 (Coefficientwise Hankel-total positivity of the GKP recurrence). The sequence $\boldsymbol{P}=\left(P_{n}(x ; \boldsymbol{\mu})\right)_{n \geqslant 0}$ of row-generating polynomials of the GKP recurrence is coefficientwise Hankel-totally positive, jointly in all seven indeterminates $x$ and $\alpha, \beta, \gamma, \alpha^{\prime}, \beta^{\prime}, \gamma^{\prime}$.

This conjecture was made a few years ago by one of us [50] and was confirmed at that time up to the $8 \times 8$ Hankel matrix $\left(P_{i+j}(x ; \boldsymbol{\mu})\right)_{0 \leqslant i, j \leqslant 7} \cdot{ }^{11}$

In fact, a slightly stronger conjecture appears to be true: in place of the usual GKP recurrence

$$
T(n, k)=(\alpha n+\beta k+\gamma) T(n-1, k)+\left(\alpha^{\prime} n+\beta^{\prime} k+\gamma^{\prime}\right) T(n-1, k-1),
$$

we can write instead

$$
T(n, k)=[\widetilde{\alpha}(n-1)+\widetilde{\beta} k+\widetilde{\gamma}] T(n-1, k)+\left[\widetilde{\alpha}^{\prime}(n-1)+\widetilde{\beta}^{\prime}(k-1)+\widetilde{\gamma}^{\prime}\right] T(n-1, k-1),
$$

which is equivalent to (6.13) with

$$
\alpha=\widetilde{\alpha}, \quad \beta=\widetilde{\beta}, \quad \gamma=\widetilde{\gamma}-\widetilde{\alpha}, \quad \alpha^{\prime}=\widetilde{\alpha}^{\prime}, \quad \beta^{\prime}=\widetilde{\beta}^{\prime}, \quad \gamma^{\prime}=\widetilde{\gamma}^{\prime}-\widetilde{\alpha}^{\prime}-\widetilde{\beta}^{\prime} .
$$

Even in this new parametrization, we apparently still get coefficientwise Hankel-total positivity:

Conjecture 6.10 (Coefficientwise Hankel-total positivity of the GKP recurrence, strong version). The sequence $\boldsymbol{P}=\left(P_{n}(x ; \widetilde{\boldsymbol{\mu}})\right)_{n \geqslant 0}$ of row-generating polynomials of the recurrence (6.14) is coefficientwise Hankel-totally positive, jointly in all seven indeterminates $x$ and $\widetilde{\alpha}, \widetilde{\beta}, \widetilde{\gamma}, \widetilde{\alpha}^{\prime}, \widetilde{\beta}^{\prime}, \widetilde{\gamma}^{\prime}$

It was in fact this stronger conjecture that was confirmed [50] up to the $8 \times 8$ Hankel matrix. We have now also confirmed the coefficientwise Hankel-total positivity of orders 2 and 3 up to the $21 \times 21$ and $9 \times 9$ Hankel matrices, respectively. ${ }^{12}$

\footnotetext{
${ }^{11}$ This computation was performed in MATHEMATICA; it required approximately $1.2 \times 10^{7}$ seconds CPU time and $587 \mathrm{~GB}$ memory, on a system using Intel Xeon E7-8837 processors running at 2.67 GHz.

${ }^{12}$ For order 2, this computation was done by checking the strong log-convexity (6.19), coefficientwise in all seven indeterminates, up to $n=38$. This is equivalent [49] to the coefficientwise total positivity of order 2 of the $21 \times 21$ Hankel matrix. This computation took $1.19 \times 10^{7}$ seconds CPU time and 19 GB memory.

For order 3, this computation was done by the direct method: it took 656116 seconds CPU time and 157 GB memory.
} 
Please observe that (6.14) is exactly what we need to ensure that the matrix elements $T(n, k)$ are polynomials with nonnegative coefficients in the parameters $\widetilde{\alpha}, \widetilde{\beta}, \widetilde{\gamma}, \widetilde{\alpha}^{\prime}, \widetilde{\beta}^{\prime}, \widetilde{\gamma}^{\prime}$, since we have written $n-1$ (resp. $k-1$ ) precisely in those terms where we know that $n-1$ (resp. $k-1$ ) must be nonnegative if the corresponding term is to make a nonzero contribution. In other words, it is immediate from (6.14) that the sequence $\boldsymbol{P}=\left(P_{n}(x ; \tilde{\boldsymbol{\mu}})\right)_{n \geqslant 0}$ is coefficientwise Hankel-totally positive of order 1 in $x$ and $\widetilde{\alpha}, \widetilde{\beta}, \widetilde{\gamma}, \widetilde{\alpha}^{\prime}, \widetilde{\beta}^{\prime}, \widetilde{\gamma}^{\prime}$. But the coefficientwise Hankel-total positivity of higher order is decidedly nontrivial!

Some very weak versions of Conjectures 6.9 and 6.10 have been proven. Liu and Wang [37, Theorem 4.1 and Remark 4.2] showed that if $\alpha, \beta, \gamma, \alpha^{\prime}, \beta^{\prime}, \gamma^{\prime}$ are real numbers satisfying

$$
\begin{aligned}
& \alpha \geqslant 0, \quad \alpha+\beta \geqslant 0, \quad \alpha+\gamma \geqslant 0 \\
& \alpha^{\prime} \geqslant 0, \quad \alpha^{\prime}+\beta^{\prime} \geqslant 0, \quad \alpha^{\prime}+\beta^{\prime}+\gamma^{\prime} \geqslant 0 \\
& \beta \alpha^{\prime}-\alpha \beta^{\prime} \geqslant 0 \\
& \beta\left(\alpha^{\prime}+\beta^{\prime}\right)-\alpha \beta^{\prime} \geqslant 0 \\
& \beta\left(\alpha^{\prime}+\beta^{\prime}+\gamma^{\prime}\right)-(\alpha+\gamma) \beta^{\prime} \geqslant 0
\end{aligned}
$$

then the sequence $\boldsymbol{P}=\left(P_{n}(x ; \tilde{\boldsymbol{\mu}})\right)_{n \geqslant 0}$ is coefficientwise log-convex in $x$, i.e.

$$
P_{n}(x) P_{n+2}(x)-P_{n+1}(x)^{2} \succeq_{x} 0
$$

for all $n \geqslant 0$. This is equivalent to the coefficientwise nonnegativity (in $x$ ) of all the contiguous $2 \times 2$ minors of the Hankel matrix $\left(P_{i+j}(x ; \boldsymbol{\mu})\right)_{i, j \geqslant 0}$; it is weaker than the full coefficientwise Hankel-total positivity of order 2.

Chen, Wang and Yang [11, Theorem 2.4] showed that under the slightly different hypotheses

$$
\begin{aligned}
& \alpha \geqslant 0, \quad \beta \geqslant 0, \quad \alpha+\beta+\gamma \geqslant 0 \\
& \alpha^{\prime} \geqslant 0, \quad \beta^{\prime} \geqslant 0, \quad \alpha^{\prime}+\beta^{\prime}+\gamma^{\prime} \geqslant 0
\end{aligned}
$$

— which neither imply nor are implied by $(6.16)$ - the sequence $\boldsymbol{P}=\left(P_{n}(x ; \tilde{\boldsymbol{\mu}})\right)_{n \geqslant 0}$ is coefficientwise strongly log-convex in $x$, i.e.

$$
P_{m}(x) P_{n+2}(x)-P_{m+1}(x) P_{n+1}(x) \succeq_{x} 0
$$

for all $n \geqslant m \geqslant 0$. It can be shown [49] that the coefficientwise strong log-convexity (in $x$ ) is equivalent to the coefficientwise (in $x$ ) Hankel-total positivity of order 2.

But these results are very far from proving even the coefficientwise Hankel-total positivity of order 2 - much less the coefficientwise Hankel-total positivity of all orders in the seven indeterminates jointly. 


\subsection{A generalization: The Graham-Knuth-Patashnik-Zhu recurrence}

Inspired by a very recent paper of Zhu [68], we would like to propose the following generalization of the GKP recurrence, which we shall call the Graham-Knuth-PatashnikZhu (GKPZ) recurrence:

$$
\begin{array}{r}
T(n, k)=(\alpha n+\beta k+\gamma) T(n-1, k)+\left(\alpha^{\prime} n+\beta^{\prime} k+\gamma^{\prime}\right) T(n-1, k-1) \\
+\sigma(n-k+1) T(n-1, k-2)+\tau(k+1) T(n-1, k+1)
\end{array}
$$

for $n \geqslant 1$ and $k \in \mathbb{Z}$, with initial condition $T(0, k)=\delta_{k 0}$ and parameters $\boldsymbol{\mu}=(\alpha, \beta, \gamma$, $\left.\alpha^{\prime}, \beta^{\prime}, \gamma^{\prime}, \sigma, \tau\right)$. Please note that because the coefficient $n-k+1$ in the $T(n-1, k-2)$ term vanishes when $k=n+1$, it follows by induction on $n$ that $T(n, k)=0$ when $k>n$. Similarly, because the coefficient $k+1$ in the $T(n-1, k+1)$ term vanishes when $k=-1$, it follows that $T(n, k)=0$ when $k<0$. So the matrix $\boldsymbol{T}(\boldsymbol{\mu})=(T(n, k ; \boldsymbol{\mu}))_{n \geqslant 0, k \in \mathbb{Z}}$ remains lower-triangular even in the presence of the two new terms.

A simple computation shows that the dual array $T^{*}(n, k) \stackrel{\text { def }}{=} T(n, n-k)$ satisfies a GKPZ recurrence with parameters

$$
D \boldsymbol{\mu} \stackrel{\text { def }}{=}\left(\alpha^{\prime}+\beta^{\prime},-\beta^{\prime}, \gamma^{\prime}, \alpha+\beta,-\beta, \gamma, \tau, \sigma\right) .
$$

This is simply the GKP duality (2.5) together with an interchange of $\sigma$ and $\tau$.

For the special case $[68 \text {, eq. }(1.3)]^{13}$

$$
\boldsymbol{\mu}=\left(0, \beta, \gamma, \alpha^{\prime},-\alpha^{\prime}+\kappa \beta, \gamma^{\prime}, \kappa \alpha^{\prime}, 0\right),
$$

Zhu [68] proved a Dobiński-type formula for the row-generating polynomials $P_{n}(x)$ [68, Theorem 2.2(i)], an explicit formula for the exponential generating function [68, Theorem 2.2(ii)], a J-fraction for the ordinary generating function [68, Theorem 2.7(i)], and coefficientwise Hankel-total positivity (in $x$ ) for various further-specialized cases of the parameters $\boldsymbol{\mu}[68$, Theorem 2.7(iii)]. The egf and J-fraction can be stated as follows:

Proposition 6.11 (Zhu [68]). For the GKPZ recurrence (6.20) with parameters $\boldsymbol{\mu}=$ $\left(0, \beta, \gamma, \alpha^{\prime},-\alpha^{\prime}+\kappa \beta, \gamma^{\prime}, \kappa \alpha^{\prime}, 0\right)$ :

(a) The egf $F(x, t ; \boldsymbol{\mu})$ is of the form

$$
F(x, t)=e^{a t}\left[1-b\left(e^{c t}-1\right)\right]^{-\Delta}
$$

where either

$$
\begin{aligned}
a & =\frac{\gamma}{\beta}\left(\beta-\alpha^{\prime} x\right) \\
b & =\left(\alpha^{\prime}+\kappa \beta\right) x \beta-\alpha^{\prime} x \\
c & =\beta-\alpha^{\prime} x \\
\Delta & =\frac{\gamma}{\beta}+\frac{\gamma^{\prime}+\kappa(\beta-\gamma)}{\alpha^{\prime}+\kappa \beta}
\end{aligned}
$$

\footnotetext{
${ }^{13}$ The connection with Zhu's [68, eq. (1.3)] variables $\lambda, d, a_{1}, a_{2}, b_{1}, b_{2}$ is $\kappa=d / \lambda$ and then $a_{1}=\beta / \lambda$, $a_{2}=\gamma / \lambda, b_{1}=\alpha^{\prime}+\kappa \beta, b_{2}=\gamma^{\prime}+\kappa(\beta-\gamma)$.
} 
or alternatively

$$
\begin{aligned}
a & =-\frac{\gamma^{\prime}+\kappa(\beta-\gamma)}{\alpha^{\prime}+\kappa \beta}\left(\beta-\alpha^{\prime} x\right) \\
b & =-\frac{\beta(1+\kappa x)}{\beta-\alpha^{\prime} x} \\
c & =-\left(\beta-\alpha^{\prime} x\right) \\
\Delta & =\frac{\gamma}{\beta}+\frac{\gamma^{\prime}+\kappa(\beta-\gamma)}{\alpha^{\prime}+\kappa \beta}
\end{aligned}
$$

(b) The ogf $f(x, t ; \boldsymbol{\mu})$ has a J-type continued fraction representation in the ring $\mathbb{Z}[x, \beta, \gamma$, $\left.\alpha^{\prime}, \kappa\right][[t]]$ with coefficients

$$
\begin{aligned}
& e_{n}=(\gamma+n \beta)(1+\kappa x)+\left[\gamma^{\prime}+\kappa(\beta-\gamma)+n\left(\alpha^{\prime}+\kappa \beta\right)\right] x \\
& f_{n}=n\left[\beta \gamma^{\prime}+\gamma \alpha^{\prime}+\kappa \beta^{2}+(n-1) \beta\left(\alpha^{\prime}+\kappa \beta\right)\right] x(1+\kappa x)
\end{aligned}
$$

There is of course also a dual result to Proposition 6.11, whose statement we leave to the reader.

We remark that Zhu [68] proves the Dobiński-type formula for $P_{n}(x)$ [68, Theorem 2.2(i)] by induction on $n$; from this he deduces the formula (6.23)-(6.25) for the egf [68, Theorem 2.2(ii)] by a straightforward computation. Finally, he deduces the Jfraction (6.26) [68, Theorem 2.7(i)] from the egf by the Stieltjes-Rogers addition-formula method [63, pp. 203-207]. ${ }^{14}$

In two special cases, the GKPZ parameters (6.22) have $\sigma=\tau=0$ and thus reduce to a GKP recurrence:

1) When $\kappa=0$, the GKPZ parameters (6.22) reduce to our family 1c (Section 5.3.1), and the J-fraction (6.26) reduces to (5.19).

2) When $\alpha^{\prime}=0$, the GKPZ parameters (6.22) reduce to our family 7a (Section 5.1.1), and the J-fraction (6.26) reduces to (5.8).

It is a very interesting open problem to generalize our work in the present paper, and the proposed work in the preceding two subsections, to the GKPZ recurrence.

\footnotetext{
${ }^{14}$ Let us remark that the argument from this egf to the J-fraction for the corresponding ogf was essentially already known to Stieltjes. More precisely, as noted already in Section 3.2, Stieltjes [56, section 81] observed that the S-fraction (3.10) with $v=y$ and $u=1$ is the formal Laplace transform of the exponential generating function (3.30); or in other words, the ogf corresponding to the egf (6.23) specialized to $a=0$ has an S-fraction with coefficients $c_{2 k-1}=b c(\Delta+k-1), c_{2 k}=k(b+1) c$. This leads by contraction (Proposition 4.1) to a J-fraction with coefficients $e_{n}=b c \Delta+n c(2 b+1), f_{n}=$ $n b(b+1)(\Delta+n-1) c^{2}$. Then one restores $a$ by applying an $a$-binomial transform (Proposition 4.5), leading to a J-fraction with $e_{n}=a+b c \Delta+n c(2 b+1), f_{n}=n b(b+1)(\Delta+n-1) c^{2}$. Inserting either (6.24) or (6.25) then yields (6.26).
} 


\section{Acknowledgements}

We acknowledge useful discussions with Eduardo J.S. Villaseñor and J. Fernando Barbero. We also thank Bao-Xuan Zhu for making available a copy of his unpublished manuscript [67], and Frank Johnson for very helpful information concerning the discrete group $\mathcal{G}$. One of us (J.S.) is grateful for the hospitality of University College London, where part of this work was done. This research was supported in part by the Spanish MINECO grant FIS2014-57387-C3-3-P; by the FEDER/Ministerio de Ciencia, Innovación y Universidades-Agencia Estatal de Investigación grant FIS2017-84440-C2-2-P; by the Madrid Government (Comunidad de Madrid-Spain) under the Multiannual Agreement with UC3M in the line of Excellence of University Professors (EPUC3M23) and in the context of the V-PRICIT (Regional Plan for Scientific Research and Technological Innovation); and by U.K. Engineering and Physical Sciences Research Council grant $\mathrm{EP} / \mathrm{N} 025636 / 1$.

\section{A Matrix product of two GKP arrays}

In this appendix we provide more details concerning the matrix product $\boldsymbol{T}(\boldsymbol{\mu}) \boldsymbol{T}(\widehat{\boldsymbol{\mu}})$ of two GKP arrays (as well as some more general arrays), with special attention to the case in which one of the two arrays is the $\xi$-binomial matrix $B_{\xi}$ defined in (4.6). Some of these results can be found in the very interesting (but apparently little-known) thesis of Théorêt [57], whose approach we largely follow in Section A.1. A few of our results are also in Spivey [52], but it seems to us that our formulations and proofs are simpler.

\section{A.1 General results}

It is convenient to start from the more general "binomial-like" recurrence introduced in Section 2.3. We have [57, p. 11, proof of Proposition 1.1.2] [59, p. 199]:

Proposition A.1 (Matrix product of two "binomial-like" arrays). Let $\boldsymbol{A}=(A(n, k))_{0 \leqslant k \leqslant n}$ and $\boldsymbol{B}=(B(n, k))_{0 \leqslant k \leqslant n}$ be triangular arrays defined by the recurrences

$$
\begin{aligned}
& A(n, k)=a_{n, k} A(n-1, k)+a_{n, k}^{\prime} A(n-1, k-1) \\
& B(n, k)=b_{n, k} B(n-1, k)+b_{n, k}^{\prime} B(n-1, k-1)
\end{aligned}
$$

for $n \geqslant 1$, with initial conditions $A(0, k)=B(0, k)=\delta_{k 0}$. Then

$$
\begin{aligned}
\sum_{j=0}^{n} A(n, j) B(j, k)= & \sum_{j=0}^{n-1}\left(a_{n, j}+a_{n, j+1}^{\prime} b_{j+1, k}\right) A(n-1, j) B(j, k) \\
& +\sum_{j=0}^{n-1} a_{n, j+1}^{\prime} b_{j+1, k}^{\prime} A(n-1, j) B(j, k-1)+\delta_{n 0} \delta_{k 0} .
\end{aligned}
$$


Proof. The formula obviously holds for $n=0$. For $n \geqslant 1$ we have

$$
\begin{aligned}
& \sum_{j=0}^{n} A(n, j) B(j, k) \\
& =\sum_{j=0}^{n-1} a_{n, j} A(n-1, j) B(j, k) \\
& \quad+\sum_{j=1}^{n} a_{n, j}^{\prime} A(n-1, j-1)\left[b_{j, k} B(j-1, k)+b_{j, k}^{\prime} B(j-1, k-1)\right] \\
& =\sum_{j=0}^{n-1} a_{n, j} A(n-1, j) B(j, k) \\
& \quad+\sum_{j=0}^{n-1} a_{n, j+1}^{\prime} A(n-1, j)\left[b_{j+1, k} B(j, k)+b_{j+1, k}^{\prime} B(j, k-1)\right] .
\end{aligned}
$$

Grouping terms gives (A.2).

Proposition A.1 does not in general give a recurrence for the matrix product $\boldsymbol{C}=\boldsymbol{A} \boldsymbol{B}$, because the coefficients on the right-hand side of (A.2) depend in general on $j$ (not just on $n$ and $k$ ). However, this dependence is eliminated whenever $a_{n, k}$ and $a_{n, k}^{\prime}$ depend only on $n$ and also $b_{n, k}$ and $b_{n, k}^{\prime}$ depend only on $k$. In this case we have [57, p. 11, Exemple 1] [7, proof of Theorem 4.8] [40, Theorem 10]:

Corollary A.2. Let $\boldsymbol{A}=(A(n, k))_{0 \leqslant k \leqslant n}$ and $\boldsymbol{B}=(B(n, k))_{0 \leqslant k \leqslant n}$ be triangular arrays defined by the recurrences

$$
\begin{aligned}
& A(n, k)=a_{n} A(n-1, k)+a_{n}^{\prime} A(n-1, k-1) \\
& B(n, k)=b_{k} B(n-1, k)+b_{k}^{\prime} B(n-1, k-1)
\end{aligned}
$$

for $n \geqslant 1$, with initial conditions $A(0, k)=B(0, k)=\delta_{k 0}$. Then the matrix product $\boldsymbol{C}=\boldsymbol{A} \boldsymbol{B}$ satisfies the recurrence

$$
C(n, k)=\left(a_{n}+a_{n}^{\prime} b_{k}\right) C(n-1, k)+a_{n}^{\prime} b_{k}^{\prime} C(n-1, k-1)
$$

for $n \geqslant 1$, with initial condition $C(0, k)=\delta_{k 0}$.

In fact, Théorêt [57, p. 13, Corollaire 1.1.3] exhibits a more general situation in which the coefficients on the right-hand side of (A.2) are independent of $j$ :

Corollary A.3. Let $\boldsymbol{A}=(A(n, k))_{0 \leqslant k \leqslant n}$ and $\boldsymbol{B}=(B(n, k))_{0 \leqslant k \leqslant n}$ be triangular arrays defined by the recurrences

$$
\begin{aligned}
A(n, k) & =\left(\alpha_{n}+\beta_{n} \gamma_{k}\right) A(n-1, k)+\beta_{n} \delta_{k} A(n-1, k-1) \\
B(n, k) & =\frac{\phi_{k}-\gamma_{n-1}}{\delta_{n}} B(n-1, k)+\frac{\psi_{k}}{\delta_{n}} B(n-1, k-1)
\end{aligned}
$$


for $n \geqslant 1$, with initial conditions $A(0, k)=B(0, k)=\delta_{k 0}$. Then the matrix product $\boldsymbol{C}=\boldsymbol{A} \boldsymbol{B}$ satisfies the recurrence

$$
C(n, k)=\left(\alpha_{n}+\beta_{n} \phi_{k}\right) C(n-1, k)+\beta_{n} \psi_{k} C(n-1, k-1)
$$

for $n \geqslant 1$, with initial condition $C(0, k)=\delta_{k 0}$.

Proof. Apply Proposition A.1.

\section{Remarks.}

1. Note that the coefficients $\gamma$ and $\delta$ occur with both $n$ (or $n-1)$ and $k$ as subscripts, while $\alpha$ and $\beta$ occur only with $n$, and $\phi$ and $\psi$ only with $k$. Note also that $\boldsymbol{\delta}$ is trivially eliminated from the matrix product $\boldsymbol{C}=\boldsymbol{A} \boldsymbol{B}$ as a consequence of the rescaling lemma (Lemma 2.1). The elimination of $\gamma$ is, however, less obvious.

2. Théorêt proves [57, pp. 13-14, Remarque 1] that (A.6) is the most general pair of "binomial-like" recurrences (with nonvanishing coefficients $a_{n, k}, a_{n, k}^{\prime}, b_{n, k}, b_{n, k}^{\prime}$ ) in which the coefficients on the right-hand side of (A.2) are independent of $j$. However, he also shows later [57, section 2.6] some other cases in which the matrix product of two binomial-like arrays is binomial-like, using a sufficient condition that is different from Proposition A.1.

Specializing Proposition A.1 to the GKP recurrence

$$
\begin{array}{ll}
a_{n, k}=\alpha n+\beta k+\gamma, & a_{n, k}^{\prime}=\alpha^{\prime} n+\beta^{\prime} k+\gamma^{\prime} \\
b_{n, k}=\widehat{\alpha} n+\widehat{\beta} k+\widehat{\gamma}, & b_{n, k}^{\prime}=\widehat{\alpha}^{\prime} n+\widehat{\beta}^{\prime} k+\widehat{\gamma}^{\prime}
\end{array}
$$

we obtain [52, Corollary 5]:

Proposition A.4 (Matrix product of two GKP arrays). Let $\boldsymbol{A}=(A(n, k))_{0 \leqslant k \leqslant n}$ and $\boldsymbol{B}=(B(n, k))_{0 \leqslant k \leqslant n}$ be triangular arrays defined by the recurrences

$$
\begin{aligned}
& A(n, k)=(\alpha n+\beta k+\gamma) A(n-1, k)+\left(\alpha^{\prime} n+\beta^{\prime} k+\gamma^{\prime}\right) A(n-1, k-1) \\
& B(n, k)=(\widehat{\alpha} n+\widehat{\beta} k+\widehat{\gamma}) B(n-1, k)+\left(\widehat{\alpha}^{\prime} n+\widehat{\beta}^{\prime} k+\widehat{\gamma}^{\prime}\right) B(n-1, k-1)
\end{aligned}
$$

for $n \geqslant 1$, with initial conditions $A(0, k)=B(0, k)=\delta_{k 0}$. Then

$$
\begin{aligned}
\sum_{j=0}^{n} A(n, j) & B(j, k)=\sum_{j=0}^{n-1} A(n-1, j) B(j, k) \\
\times & {\left[(\alpha n+\beta j+\gamma)+\left(\alpha^{\prime} n+\beta^{\prime}(j+1)+\gamma^{\prime}\right)(\widehat{\alpha}(j+1)+\widehat{\beta} k+\widehat{\gamma})\right] } \\
& +\sum_{j=0}^{n-1} A(n-1, j) B(j, k-1)\left(\alpha^{\prime} n+\beta^{\prime}(j+1)+\gamma^{\prime}\right)\left(\widehat{\alpha}^{\prime}(j+1)+\widehat{\beta}^{\prime} k+\widehat{\gamma}^{\prime}\right) \\
& +\delta_{n 0} \delta_{k 0} .
\end{aligned}
$$


Let us remark that Spivey [52] used the variant notation (6.14) for the GKP recurrence; restating his result [52, Corollary 5] in terms of the standard notation (1.1) and simplifying the expression slightly yields (A.10).

And specializing further to $\beta=\beta^{\prime}=\widehat{\alpha}=\widehat{\alpha}^{\prime}=0$, we obtain the GKP special case of Corollary A.2:

Corollary A.5. Let $\boldsymbol{A}=(A(n, k))_{0 \leqslant k \leqslant n}$ and $\boldsymbol{B}=(B(n, k))_{0 \leqslant k \leqslant n}$ be triangular arrays defined by the recurrences

$$
\begin{aligned}
& A(n, k)=(\alpha n+\gamma) A(n-1, k)+\left(\alpha^{\prime} n+\gamma^{\prime}\right) A(n-1, k-1) \\
& B(n, k)=(\widehat{\beta} k+\widehat{\gamma}) B(n-1, k)+\left(\widehat{\beta}^{\prime} k+\widehat{\gamma}^{\prime}\right) B(n-1, k-1)
\end{aligned}
$$

for $n \geqslant 1$, with initial conditions $A(0, k)=B(0, k)=\delta_{k 0}$. Then the matrix product $\boldsymbol{C}=\boldsymbol{A} \boldsymbol{B}$ satisfies the recurrence

$$
\begin{array}{r}
C(n, k)=\left[(\alpha n+\gamma)+\left(\alpha^{\prime} n+\gamma^{\prime}\right)(\widehat{\beta} k+\widehat{\gamma})\right] C(n-1, k) \\
+\left(\alpha^{\prime} n+\gamma^{\prime}\right)\left(\widehat{\beta}^{\prime} k+\widehat{\gamma}^{\prime}\right) C(n-1, k-1)
\end{array}
$$

for $n \geqslant 1$, with initial condition $C(0, k)=\delta_{k 0}$.

Of course, the binomial-like recurrence (A.12) need not in general be of GKP form, due to the possibility of coefficients involving the product $n k$. But these terms vanish if, in addition, either $\alpha^{\prime}=0$ or $\widehat{\beta}=\widehat{\beta}^{\prime}=0$ :

Corollary A.6. Let $\boldsymbol{A}=(A(n, k))_{0 \leqslant k \leqslant n}$ and $\boldsymbol{B}=(B(n, k))_{0 \leqslant k \leqslant n}$ be triangular arrays defined by the recurrences

$$
\begin{aligned}
& A(n, k)=(\alpha n+\gamma) A(n-1, k)+\left(\alpha^{\prime} n+\gamma^{\prime}\right) A(n-1, k-1) \\
& B(n, k)=(\widehat{\beta} k+\widehat{\gamma}) B(n-1, k)+\left(\widehat{\beta}^{\prime} k+\widehat{\gamma}^{\prime}\right) B(n-1, k-1)
\end{aligned}
$$

for $n \geqslant 1$, with initial conditions $A(0, k)=B(0, k)=\delta_{k 0}$. Assume further that either $\alpha^{\prime}=0$ or $\widehat{\beta}=\widehat{\beta}^{\prime}=0$. Then the matrix product $\boldsymbol{C}=\boldsymbol{A B}$ satisfies the GKP recurrence

$$
\begin{array}{r}
C(n, k)=\left[\left(\alpha+\alpha^{\prime} \widehat{\gamma}\right) n+\gamma^{\prime} \widehat{\beta} k+\left(\gamma+\gamma^{\prime} \widehat{\gamma}\right)\right] C(n-1, k) \\
+\left(\alpha^{\prime} \widehat{\gamma}^{\prime} n+\gamma^{\prime} \widehat{\beta}^{\prime} k+\gamma^{\prime} \widehat{\gamma}^{\prime}\right) C(n-1, k-1)
\end{array}
$$

for $n \geqslant 1$, with initial condition $C(0, k)=\delta_{k 0}$.

Remark. In Corollary A.6, the matrix $\boldsymbol{B}$ is precisely the matrix $\boldsymbol{T}$ of family 7 a (with hats inserted on the parameters); and if we further specialize to $\left(\alpha, \gamma, \alpha^{\prime}, \gamma^{\prime}\right)=(0, \xi, 0,1)$, then $\boldsymbol{A}$ is the $\xi$-binomial matrix $B_{\xi}$. In this case the matrix product $B_{\xi} \boldsymbol{T}$ satisfies a GKP recurrence (A.14) of the same form (A.13b) but with $\widehat{\gamma} \rightarrow \widehat{\gamma}+\xi$. This gives the promised direct proof that family $7 \mathrm{a}$ is the $\gamma$-binomial transform of family $3 \mathrm{a}$ (Proposition 5.1).

Alternatively, we can obtain GKP recurrences from Corollary A.3 by setting

$$
\alpha_{n}=\alpha n+\gamma, \quad \beta_{n}=\gamma^{\prime}, \quad \gamma_{k}=\left(\beta / \gamma^{\prime}\right) k, \quad \delta_{k}=1, \quad \phi_{k}=\widehat{\beta} k, \quad \psi_{k}=\widehat{\beta}^{\prime} k+\widehat{\gamma}^{\prime} .
$$

We then have: 
Corollary A.7. Let $\boldsymbol{A}=(A(n, k))_{0 \leqslant k \leqslant n}$ and $\boldsymbol{B}=(B(n, k))_{0 \leqslant k \leqslant n}$ be triangular arrays defined by the recurrences

$$
\begin{aligned}
& A(n, k)=(\alpha n+\beta k+\gamma) A(n-1, k)+\gamma^{\prime} A(n-1, k-1) \\
& B(n, k)=\left(-\frac{\beta}{\gamma^{\prime}} n+\widehat{\beta} k+\frac{\beta}{\gamma^{\prime}}\right) B(n-1, k)+\left(\widehat{\beta}^{\prime} k+\widehat{\gamma}^{\prime}\right) B(n-1, k-1)
\end{aligned}
$$

for $n \geqslant 1$, with initial conditions $A(0, k)=B(0, k)=\delta_{k 0}$. Then the matrix product $\boldsymbol{C}=\boldsymbol{A} \boldsymbol{B}$ satisfies the recurrence

$$
C(n, k)=\left(\alpha n+\gamma^{\prime} \widehat{\beta} k+\gamma\right) C(n-1, k)+\gamma^{\prime}\left(\widehat{\beta}^{\prime} k+\widehat{\gamma}^{\prime}\right) C(n-1, k-1)
$$

for $n \geqslant 1$, with initial condition $C(0, k)=\delta_{k 0}$.

Going back to Proposition A.4, another way to handle the $j$-dependent terms - rather than assuming that they are nonexistent, i.e. $\beta=\beta^{\prime}=\widehat{\alpha}=\widehat{\alpha}^{\prime}=0-$ is to transform them away by employing identities to convert the $j$-dependence to $n$ - or $k$-dependence. This works in some special cases, as a result of the following simple fact:

Lemma A.8 (Identities for nearly-binomial matrices).

(a) If the matrix $\boldsymbol{T}$ is of the form

$$
T(n, k)=\left(\begin{array}{l}
n \\
k
\end{array}\right) \gamma^{n-k} f(k)
$$

for some function $f(k)$, then for every integer $r \geqslant 0$,

$$
(n-k)^{\underline{r}} T(n, k)=\gamma^{r} n^{\underline{r}} T(n-r, k) .
$$

[Here $x^{\underline{\underline{r}}} \stackrel{\text { def }}{=} x(x-1) \cdots(x-r+1)$.] This holds in particular whenever $\boldsymbol{T}=\boldsymbol{T}(\boldsymbol{\mu})$ with $\boldsymbol{\mu}=\left(0,0, \gamma, 0, \beta^{\prime}, \gamma^{\prime}\right)$, in which case

$$
T(n, k)=\left(\begin{array}{l}
n \\
k
\end{array}\right) \gamma^{n-k} \prod_{j=1}^{k}\left(\gamma^{\prime}+j \beta^{\prime}\right) .
$$

(b) If the matrix $\boldsymbol{T}$ is of the form

$$
T(n, k)=\left(\begin{array}{l}
n \\
k
\end{array}\right)\left(\gamma^{\prime}\right)^{k} g(n-k)
$$

for some function $g(n-k)$, then for every integer $r \geqslant 0$,

$$
k^{\underline{r}} T(n, k)=\left(\gamma^{\prime}\right)^{r} n^{\underline{r}} T(n-r, k-r) .
$$

This holds in particular whenever $\boldsymbol{T}=\boldsymbol{T}(\boldsymbol{\mu})$ with $\boldsymbol{\mu}=\left(\alpha,-\alpha, \gamma, 0,0, \gamma^{\prime}\right)$, in which case

$$
T(n, k)=\left(\begin{array}{l}
n \\
k
\end{array}\right)\left(\gamma^{\prime}\right)^{k} \prod_{j=1}^{n-k}(\gamma+j \alpha) .
$$


Proof. An easy computation.

We next discuss some special cases of Proposition A.4 (or more generally, Proposition A.1) where Lemma A.8 can be used to remove the $j$-dependence; we divide these according as the special matrix (i.e. the one to which Lemma A.8 is applied) is on the left (Section A.2) or on the right (Section A.3).

\section{A.2 Special matrix on the left}

We distinguish two cases, according as we apply part (a) or (b) of Lemma A.8 to the matrix $\boldsymbol{A}$ :

Case 1: $\boldsymbol{\alpha}=\boldsymbol{\beta}=\boldsymbol{\alpha}^{\prime}=\mathbf{0}$. When $\boldsymbol{A}$ is given by a GKP recurrence with $\alpha=\beta=\alpha^{\prime}=0$, we can handle a matrix $\boldsymbol{B}$ that is more general than a GKP recurrence: namely, it can be given by a binomial-like recurrence (A.1b) in which $b_{n, k}$ and $b_{n, k}^{\prime}$ are affine in $n$ but with coefficients depending in an arbitrary way on $k$ :

$$
a_{n, k}=\gamma, \quad a_{n, k}^{\prime}=\beta^{\prime} k+\gamma^{\prime}, \quad b_{n, k}=\widehat{\alpha}_{k} n+\widehat{\gamma}_{k}, \quad b_{n, k}^{\prime}=\widehat{\alpha}_{k}^{\prime} n+\widehat{\gamma}_{k}^{\prime} .
$$

We then start from Proposition A.1 with the specializations (A.24), and isolate the terms proportional to $j$ or $j^{2}$ : the matrix $\boldsymbol{C}=\boldsymbol{A} \boldsymbol{B}$ satisfies

$$
\begin{aligned}
C(n, k)= & {\left[\left(\beta^{\prime}+\gamma^{\prime}\right)\left(\widehat{\alpha}_{k}+\widehat{\gamma}_{k}\right)+\gamma\right] C(n-1, k) } \\
& +\left(\beta^{\prime}+\gamma^{\prime}\right)\left(\widehat{\alpha}_{k}^{\prime}+\widehat{\gamma}_{k}^{\prime}\right) C(n-1, k-1) \\
& +\left[\beta^{\prime}\left(\widehat{\alpha}_{k}+\widehat{\gamma}_{k}\right)+\left(\beta^{\prime}+\gamma^{\prime}\right) \widehat{\alpha}_{k}\right] \sum_{j=1}^{n-1} j A(n-1, j) B(j, k) \\
& +\beta^{\prime} \widehat{\alpha}_{k} \sum_{j=1}^{n-1} j^{2} A(n-1, j) B(j, k) \\
& +\left[\beta^{\prime}\left(\widehat{\alpha}_{k}^{\prime}+\widehat{\gamma}_{k}^{\prime}\right)+\left(\beta^{\prime}+\gamma^{\prime}\right) \widehat{\alpha}_{k}^{\prime}\right] \sum_{j=1}^{n-1} j A(n-1, j) B(j, k-1) \\
& +\beta^{\prime} \widehat{\alpha}_{k}^{\prime} \sum_{j=1}^{n-1} j^{2} A(n-1, j) B(j, k-1)
\end{aligned}
$$

for $n \geqslant 1$. Now apply Lemma A.8(a) to the matrix $\boldsymbol{A}$ : from (A.19) with $r=1,2$ we deduce

$$
\begin{aligned}
j A(n, j) & =n A(n, j)-\gamma n A(n-1, j) \\
j^{2} A(n, j) & =n^{2} A(n, j)-\gamma n(2 n-1) A(n-1, j)+\gamma^{2} n(n-1) A(n-2, j)
\end{aligned}
$$

Inserting (A.26a,b) with $n \rightarrow n-1$ on the right-hand side of (A.25), we obtain: 
Corollary A.9. Let $\boldsymbol{A}=(A(n, k))_{0 \leqslant k \leqslant n}$ and $\boldsymbol{B}=(B(n, k))_{0 \leqslant k \leqslant n}$ be triangular arrays defined by the recurrences

$$
\begin{aligned}
& A(n, k)=\gamma A(n-1, k)+\left(\beta^{\prime} k+\gamma^{\prime}\right) A(n-1, k-1) \\
& B(n, k)=\left(\widehat{\alpha}_{k} n+\widehat{\gamma}_{k}\right) B(n-1, k)+\left(\widehat{\alpha}_{k}^{\prime} n+\widehat{\gamma}_{k}^{\prime}\right) B(n-1, k-1)
\end{aligned}
$$

for $n \geqslant 1$, with initial conditions $A(0, k)=B(0, k)=\delta_{k 0}$. Then the matrix product $\boldsymbol{C}=\boldsymbol{A} \boldsymbol{B}$ satisfies the recurrence

$$
\begin{aligned}
C(n, k)= & c_{1} C(n-1, k)+c_{2} C(n-1, k-1) \\
& +c_{3} C(n-2, k)+c_{4} C(n-2, k-1) \\
& +c_{5} C(n-3, k)+c_{6} C(n-3, k-1)
\end{aligned}
$$

for $n \geqslant 1$, with initial condition $C(0, k)=\delta_{k 0}$, where

$$
\begin{aligned}
& c_{1}=\left(\beta^{\prime} n+\gamma^{\prime}\right)\left(\widehat{\alpha}_{k} n+\widehat{\gamma}_{k}\right)+\gamma \\
& c_{2}=\left(\beta^{\prime} n+\gamma^{\prime}\right)\left(\widehat{\alpha}_{k}^{\prime} n+\widehat{\gamma}_{k}^{\prime}\right) \\
& c_{3}=-(n-1) \gamma\left[\gamma^{\prime} \widehat{\alpha}_{k}+\beta^{\prime}\left(\widehat{\gamma}_{k}+(2 n-1) \widehat{\alpha}_{k}\right)\right] \\
& c_{4}=-(n-1) \gamma\left[\gamma^{\prime} \widehat{\alpha}_{k}^{\prime}+\beta^{\prime}\left(\widehat{\gamma}_{k}^{\prime}+(2 n-1) \widehat{\alpha}_{k}^{\prime}\right)\right] \\
& c_{5}=(n-1)(n-2) \gamma^{2} \beta^{\prime} \widehat{\alpha}_{k} \\
& c_{6}=(n-1)(n-2) \gamma^{2} \beta^{\prime} \widehat{\alpha}_{k}^{\prime}
\end{aligned}
$$

This is rather complicated, but it simplifies significantly when also $\beta^{\prime}=0$, in which case $\boldsymbol{A}$ is a rescaled binomial: $A(n, k)=\left(\begin{array}{l}n \\ k\end{array}\right) \gamma^{n-k}\left(\gamma^{\prime}\right)^{k}$. Normalizing to $\gamma^{\prime}=1$ and writing $\gamma=\xi$, then $\boldsymbol{A}$ becomes the $\xi$-binomial matrix $B_{\xi}$ defined in (4.6), and Corollary A.9 becomes:

Corollary A.10. Let $\boldsymbol{B}=(B(n, k))_{0 \leqslant k \leqslant n}$ be a triangular array defined by the recurrence

$$
B(n, k)=\left(\widehat{\alpha}_{k} n+\widehat{\gamma}_{k}\right) B(n-1, k)+\left(\widehat{\alpha}_{k}^{\prime} n+\widehat{\gamma}_{k}^{\prime}\right) B(n-1, k-1)
$$

for $n \geqslant 1$, with initial condition $B(0, k)=\delta_{k 0}$. Then the matrix product $\boldsymbol{C}=B_{\xi} \boldsymbol{B}$ satisfies the recurrence

$$
\begin{aligned}
C(n, k)= & \left(\widehat{\alpha}_{k} n+\widehat{\gamma}_{k}+\xi\right) C(n-1, k)+\left(\widehat{\alpha}_{k}^{\prime} n+\widehat{\gamma}_{k}^{\prime}\right) C(n-1, k-1) \\
& -(n-1) \xi\left[\widehat{\alpha}_{k} C(n-2, k)+\widehat{\alpha}_{k}^{\prime} C(n-2, k-1)\right]
\end{aligned}
$$

for $n \geqslant 1$, with initial condition $C(0, k)=\delta_{k 0}$.

When also $\widehat{\alpha}_{k}=\widehat{\alpha}_{k}^{\prime}=0$ for all $k$, the terms $C(n-2, k)$ and $C(n-2, k-1)$ disappear, and we have a special case of Corollary A.2.

Specializing Corollary A.10 to the GKP case

$$
\widehat{\alpha}_{k}=\widehat{\alpha}, \quad \widehat{\gamma}_{k}=\widehat{\beta} k+\widehat{\gamma}, \quad \widehat{\alpha}_{k}^{\prime}=\widehat{\alpha}^{\prime}, \quad \widehat{\gamma}_{k}^{\prime}=\widehat{\beta}^{\prime} k+\widehat{\gamma}^{\prime},
$$

we get: 
Corollary A.11. Let $\boldsymbol{B}=(B(n, k))_{0 \leqslant k \leqslant n}$ be a triangular array defined by the recurrence

$$
B(n, k)=(\widehat{\alpha} n+\widehat{\beta} k+\widehat{\gamma}) B(n-1, k)+\left(\widehat{\alpha}^{\prime} n+\widehat{\beta}^{\prime} k+\widehat{\gamma}^{\prime}\right) B(n-1, k-1)
$$

for $n \geqslant 1$, with initial condition $B(0, k)=\delta_{k 0}$. Then the matrix product $\boldsymbol{C}=B_{\xi} \boldsymbol{B}$ satisfies the recurrence

$$
\begin{aligned}
C(n, k)= & (\widehat{\alpha} n+\widehat{\beta} k+\widehat{\gamma}+\xi) C(n-1, k)+\left(\widehat{\alpha}^{\prime} n+\widehat{\beta}^{\prime} k+\widehat{\gamma}^{\prime}\right) C(n-1, k-1) \\
& -(n-1) \xi\left[\widehat{\alpha} C(n-2, k)+\widehat{\alpha}^{\prime} C(n-2, k-1)\right]
\end{aligned}
$$

for $n \geqslant 1$, with initial condition $C(0, k)=\delta_{k 0}$.

When also $\widehat{\alpha}=\widehat{\alpha}^{\prime}=0$, the terms $C(n-2, k)$ and $C(n-2, k-1)$ disappear, and we are back in the situation discussed in the Remark after Corollary A.6.

The form (A.34) of the output matrix in Corollary A.10 suggests that one consider, as input, a triangular array $\boldsymbol{B}=(B(n, k))_{0 \leqslant k \leqslant n}$ defined by a similar recurrence. Then we can obtain, using essentially the same proof as for Corollary A.10, the following more general result:

Corollary A.12. Let $\boldsymbol{B}=(B(n, k))_{0 \leqslant k \leqslant n}$ be a triangular array defined by the recurrence

$$
\begin{aligned}
B(n, k)=\left(\widehat{\alpha}_{k} n+\widehat{\gamma}_{k}\right) B(n-1, k)+\left(\widehat{\alpha}_{k}^{\prime} n+\widehat{\gamma}_{k}^{\prime}\right) B(n-1, k-1) \\
+(n-1) \widehat{\delta}_{k} B(n-2, k)+(n-1) \widehat{\delta}_{k}^{\prime} B(n-2, k-1)
\end{aligned}
$$

for $n \geqslant 1$, with initial condition $B(0, k)=\delta_{k 0}$. Then the matrix product $\boldsymbol{C}=B_{\xi} \boldsymbol{B}$ satisfies the recurrence

$$
\begin{aligned}
C(n, k) & =\left(\widehat{\alpha}_{k} n+\widehat{\gamma}_{k}+\xi\right) C(n-1, k)+\left(\widehat{\alpha}_{k}^{\prime} n+\widehat{\gamma}_{k}^{\prime}\right) C(n-1, k-1) \\
& +(n-1)\left(\widehat{\delta}_{k}-\xi \widehat{\alpha}_{k}\right) C(n-2, k)+(n-1)\left(\widehat{\delta}_{k}^{\prime}-\xi \widehat{\alpha}_{k}^{\prime}\right) C(n-2, k-1)
\end{aligned}
$$

for $n \geqslant 1$, with initial condition $C(0, k)=\delta_{k 0}$.

Proof. The matrix $\boldsymbol{C}=B_{\xi} \boldsymbol{B}$ satisfies

$$
\begin{aligned}
C(n, k) & =\sum_{j=0}^{n} B_{\xi}(n, j) B(j, k) \\
& =\sum_{j=0}^{n}\left[\xi B_{\xi}(n-1, j)+B_{\xi}(n-1, j-1)\right] B(j, k) \\
& =\xi C(n-1, k)+\sum_{j=1}^{n} B_{\xi}(n-1, j-1) B(j, k) .
\end{aligned}
$$


Now insert the recurrence (A.35) for $B(j, k)$. The first two terms on the right-hand side produce the terms given in Corollary A.10. So we only need to deal with the last two terms on the right-hand side. The contribution of the first one is

$$
\begin{aligned}
\widehat{\delta}_{k} \sum_{j=1}^{n}(j-1) & B_{\xi}(n-1, j-1) B(j-2, k) \\
& =\widehat{\delta}_{k} \sum_{j=0}^{n-1} j B_{\xi}(n-1, j) B(j-1, k) \\
& =\widehat{\delta}_{k}(n-1) C(n-2, k) \quad[\text { using (A.22) with } r=1] .
\end{aligned}
$$

Similarly, the contribution of the second term is $\widehat{\delta}_{k}^{\prime}(n-1) C(n-2, k-1)$. These two contributions plus those given by Corollary A.10 give the claimed formula (A.36).

Case 2: $\boldsymbol{\beta}=-\boldsymbol{\alpha}, \boldsymbol{\alpha}^{\prime}=\boldsymbol{\beta}^{\prime}=\mathbf{0}$. As in Case 1, we can handle a matrix $\boldsymbol{B}$ given by a binomial-like recurrence (A.1b) in which $b_{n, k}$ and $b_{n, k}^{\prime}$ are affine in $n$ but with coefficients depending in an arbitrary way on $k$ :

$$
a_{n, k}=\alpha(n-k)+\gamma, \quad a_{n, k}^{\prime}=\gamma^{\prime}, \quad b_{n, k}=\widehat{\alpha}_{k} n+\widehat{\gamma}_{k}, \quad b_{n, k}^{\prime}=\widehat{\alpha}_{k}^{\prime} n+\widehat{\gamma}_{k}^{\prime} .
$$

We then insert the specializations (A.39) into Proposition A.1 and isolate the terms proportional to $j$ (note that the terms proportional to $j^{2}$ vanish in this case): the matrix $\boldsymbol{C}=\boldsymbol{A} \boldsymbol{B}$ satisfies

$$
\begin{aligned}
C(n, k)= & {\left[\alpha n+\gamma+\gamma^{\prime}\left(\widehat{\alpha}_{k}+\widehat{\gamma}_{k}\right)\right] C(n-1, k)+\gamma^{\prime}\left(\widehat{\alpha}_{k}^{\prime}+\widehat{\gamma}_{k}^{\prime}\right) C(n-1, k-1) } \\
& +\left(\gamma^{\prime} \widehat{\alpha}_{k}-\alpha\right) \sum_{j=1}^{n-1} j A(n-1, j) B(j, k)+\gamma^{\prime} \widehat{\alpha}_{k}^{\prime} \sum_{j=1}^{n-1} j A(n-1, j) B(j, k-1)
\end{aligned}
$$

for $n \geqslant 1$. Now apply Lemma A.8(b) with $r=1$ to the matrix $\boldsymbol{A}$ :

$$
j A(n-1, j)=\gamma^{\prime}(n-1) A(n-2, j-1) .
$$

And apply the recurrence (A.9b) to $B(j, k)$ and $B(j, k-1)$ in order to convert $j$ to $j-1$ (so that we can again get a matrix product $\boldsymbol{A B}$ ). The last two terms of (A.40) become

$$
\begin{aligned}
& \gamma^{\prime}(n-1)\left(\gamma^{\prime} \widehat{\alpha}_{k}-\alpha\right) \sum_{j=1}^{n-1} A(n-2, j-1) \times \\
& \quad\left[\left(\widehat{\alpha}_{k} j+\widehat{\gamma}_{k}\right) B(j-1, k)+\left(\widehat{\alpha}_{k}^{\prime} j+\widehat{\gamma}_{k}^{\prime}\right) B(j-1, k-1)\right] . \\
& +\left(\gamma^{\prime}\right)^{2}(n-1) \widehat{\alpha}_{k}^{\prime} \sum_{j=1}^{n-1} A(n-2, j-1) \times \\
& \quad\left[\left(\widehat{\alpha}_{k-1} j+\widehat{\gamma}_{k-1}\right) B(j-1, k-1)+\left(\widehat{\alpha}_{k-1}^{\prime} j+\widehat{\gamma}_{k-1}^{\prime}\right) B(j-1, k-2)\right] .
\end{aligned}
$$


The appearance here of the terms $\widehat{\alpha}_{i} j$ and $\widehat{\alpha}_{i}^{\prime} j$ (for $i=k, k-1$ ) would lead in general to an infinite regress of $j$-dependence, but this $j$-dependence disappears if $\widehat{\alpha}_{k}^{\prime}=0$ for all $k$ and moreover either (a) $\gamma^{\prime} \widehat{\alpha}_{k}=0$ for all $k$ or (b) $\gamma^{\prime} \widehat{\alpha}_{k}=\alpha$ for all $k$. We then have:

Corollary A.13. Let $\boldsymbol{A}=(A(n, k))_{0 \leqslant k \leqslant n}$ and $\boldsymbol{B}=(B(n, k))_{0 \leqslant k \leqslant n}$ be triangular arrays defined by the recurrences

$$
\begin{aligned}
& A(n, k)=(\alpha(n-k)+\gamma) A(n-1, k)+\gamma^{\prime} A(n-1, k-1) \\
& B(n, k)=\left(\widehat{\alpha}_{k} n+\widehat{\gamma}_{k}\right) B(n-1, k)+\widehat{\gamma}_{k}^{\prime} B(n-1, k-1)
\end{aligned}
$$

for $n \geqslant 1$, with initial conditions $A(0, k)=B(0, k)=\delta_{k 0}$. Suppose further that either $\gamma^{\prime} \widehat{\alpha}_{k}=0$ for all $k$ or $\gamma^{\prime} \widehat{\alpha}_{k}=\alpha$ for all $k$. Then the matrix product $\boldsymbol{C}=\boldsymbol{A} \boldsymbol{B}$ satisfies the recurrence

$$
\begin{aligned}
C(n, k)= & c_{1}^{\prime} C(n-1, k)+c_{2}^{\prime} C(n-1, k-1) \\
& +c_{3}^{\prime} C(n-2, k)+c_{4}^{\prime} C(n-2, k-1)
\end{aligned}
$$

for $n \geqslant 1$, with initial condition $C(0, k)=\delta_{k 0}$, where

$$
\begin{aligned}
c_{1}^{\prime} & =\alpha n+\gamma+\gamma^{\prime}\left(\widehat{\alpha}_{k}+\widehat{\gamma}_{k}\right) \\
c_{2}^{\prime} & =\gamma^{\prime} \widehat{\gamma}_{k}^{\prime} \\
c_{3}^{\prime} & =(n-1) \gamma^{\prime}\left(\gamma^{\prime} \widehat{\alpha}_{k}-\alpha\right) \widehat{\gamma}_{k} \\
c_{4}^{\prime} & =(n-1) \gamma^{\prime}\left(\gamma^{\prime} \widehat{\alpha}_{k}-\alpha\right) \widehat{\gamma}_{k}^{\prime}
\end{aligned}
$$

In particular, when $\gamma^{\prime} \widehat{\alpha}_{k}=\alpha$ for all $k$, we have $c_{3}^{\prime}=c_{4}^{\prime}=0$ and hence a binomial-like recurrence for $\boldsymbol{C}$.

The further specialization of Corollary A.13 to the GKP case (A.32) is easily written out. In particular, when $\widehat{\alpha}^{\prime}=0$ and $\gamma^{\prime} \widehat{\alpha}=\alpha$, then the recurrence for $\boldsymbol{C}$ is of GKP form.

Remarks. 1. The "suppose further" hypotheses of Corollary A.13 are satisfied when $\gamma^{\prime}=0$, but this is a trivial case: the matrix $\boldsymbol{A}$ vanishes outside the zeroth column, so that $\boldsymbol{A B}=\boldsymbol{A}$.

2. Suppose that $\widehat{\alpha}_{k}$ has a constant value $\widehat{\alpha}$ (independent of $k$ ) but that we suppress the hypothesis on $\gamma^{\prime} \widehat{\alpha}$. Then the difference between the left-hand and right-hand sides of (A.44) is, for each pair $n, k$, a polynomial in the parameters that has $\gamma^{\prime} \widehat{\alpha}\left(\gamma^{\prime} \widehat{\alpha}-\alpha\right)$ as an overall factor.

The intersection of cases 1 and 2: $\boldsymbol{\alpha}=\boldsymbol{\beta}=\boldsymbol{\alpha}^{\prime}=\boldsymbol{\beta}^{\prime}=\widehat{\boldsymbol{\alpha}}_{\boldsymbol{k}}=\widehat{\boldsymbol{\alpha}}_{\boldsymbol{k}}^{\prime}=\mathbf{0}$. Let us now compare Corollary A.9 specialized to $\beta^{\prime}=\widehat{\alpha}_{k}=\widehat{\alpha}_{k}^{\prime}=0$ with Corollary A.13 specialized 
to $\alpha=\widehat{\alpha}_{k}=0$. We obtain a recurrence of GKP type with

$$
\begin{aligned}
c_{1}=c_{1}^{\prime} & =\gamma^{\prime} \widehat{\gamma}_{k}+\gamma \\
c_{2}=c_{2}^{\prime} & =\gamma^{\prime} \widehat{\gamma}_{k}^{\prime} \\
c_{3}=c_{3}^{\prime} & =0 \\
c_{4}=c_{4}^{\prime} & =0 \\
c_{5} & =0 \\
c_{6} & =0
\end{aligned}
$$

\section{A.3 Special matrix on the right}

Once again we distinguish two cases, according as we apply part (a) or (b) of Lemma A.8 to the matrix $\boldsymbol{B}$ :

Case 3: $\widehat{\boldsymbol{\alpha}}=\widehat{\boldsymbol{\beta}}=\widehat{\boldsymbol{\alpha}}^{\prime}=\mathbf{0}$. We start from Proposition A.4 specialized to $\widehat{\alpha}=\widehat{\beta}=\widehat{\alpha}^{\prime}=$ 0 , and isolate the terms proportional to $j$ (note that the terms proportional to $j^{2}$ vanish in this case): the matrix $\boldsymbol{C}=\boldsymbol{A} \boldsymbol{B}$ satisfies

$$
\begin{aligned}
C(n, k)= & {\left[(\alpha n+\gamma)+\widehat{\gamma}\left(\alpha^{\prime} n+\beta^{\prime}+\gamma^{\prime}\right)\right] C(n-1, k) } \\
& +\left(\alpha^{\prime} n+\beta^{\prime}+\gamma^{\prime}\right)\left(\widehat{\beta}^{\prime} k+\widehat{\gamma}^{\prime}\right) C(n-1, k-1) \\
& +\left(\beta+\beta^{\prime} \widehat{\gamma}\right) \sum_{j=1}^{n-1} j A(n-1, j) B(j, k) \\
& +\beta^{\prime}\left(\widehat{\beta}^{\prime} k+\widehat{\gamma}^{\prime}\right) \sum_{j=1}^{n-1} j A(n-1, j) B(j, k-1)
\end{aligned}
$$

for $n \geqslant 1$. The next step would be to apply Lemma A.8(a) with $r=1$ to the matrix $\boldsymbol{B}$ :

$$
j B(j, k-1)=(k-1) B(j, k-1)+\widehat{\gamma} j B(j-1, k-1) .
$$

But this leads to an infinite regress of $j$-dependence (except in the degenerate case $\widehat{\gamma}=0$, which leads to a matrix $\boldsymbol{B}$ that vanishes outside the zeroth column). So we do not know how to obtain a recurrence for $\boldsymbol{C}=\boldsymbol{A} \boldsymbol{B}$ in this case.

Case $4: \widehat{\boldsymbol{\beta}}=-\widehat{\boldsymbol{\alpha}}, \widehat{\boldsymbol{\alpha}}^{\prime}=\widehat{\boldsymbol{\beta}}^{\prime}=\mathbf{0}$. Similarly to what was done in Cases 1 and 2 - but now with the roles of $\boldsymbol{A}$ and $\boldsymbol{B}$, and also $n$ and $k$, reversed - we can handle a matrix $\boldsymbol{A}$ given by a binomial-like recurrence (A.1a) in which $a_{n, k}$ and $a_{n, k}^{\prime}$ are affine in $k$ but with coefficients depending in an arbitrary way on $n$ :

$$
a_{n, k}=\beta_{n} k+\gamma_{n}, \quad a_{n, k}^{\prime}=\beta_{n}^{\prime} k+\gamma_{n}^{\prime}, \quad b_{n, k}=\widehat{\alpha}(n-k)+\widehat{\gamma}, \quad b_{n, k}^{\prime}=\widehat{\gamma}^{\prime} .
$$


We insert the specializations (A.49) into Proposition A.1 and isolate the terms proportional to $j$ or $j^{2}$ : the matrix $\boldsymbol{C}=\boldsymbol{A} \boldsymbol{B}$ satisfies

$$
\begin{aligned}
C(n, k)= & {\left[\gamma_{n}+\left(\beta_{n}^{\prime}+\gamma_{n}^{\prime}\right)(-\widehat{\alpha} k+\widehat{\alpha}+\widehat{\gamma})\right] C(n-1, k) } \\
& +\widehat{\gamma}^{\prime}\left(\beta_{n}^{\prime}+\gamma_{n}^{\prime}\right) C(n-1, k-1) \\
& +\left[\beta_{n}+\beta_{n}^{\prime}(-\widehat{\alpha} k+\widehat{\alpha}+\widehat{\gamma})+\widehat{\alpha}\left(\beta_{n}^{\prime}+\gamma_{n}^{\prime}\right)\right] \sum_{j=1}^{n-1} j A(n-1, j) B(j, k) \\
& +\beta_{n}^{\prime} \widehat{\alpha} \sum_{j=1}^{n-1} j^{2} A(n-1, j) B(j, k) \\
& +\beta_{n}^{\prime} \widehat{\gamma}^{\prime} \sum_{j=1}^{n-1} j A(n-1, j) B(j, k-1)
\end{aligned}
$$

for $n \geqslant 1$. Now apply Lemma A.8(b) in reverse to the matrix $\boldsymbol{B}$ : from (A.22) with $r=1,2$ we deduce

$$
j B(j, k)=\frac{1}{\widehat{\gamma}^{\prime}}(k+1) B(j+1, k+1)-B(j, k)
$$

and a similar but more complicated formula for $j^{2} B(j, k)$. Applying the recurrence (A.9b) to $B(j+1, k+1)$ in order to convert $j+1$ to $j$, we can rewrite (A.51) as

$$
j B(j, k)=k B(j, k)+\frac{1}{\widehat{\gamma}^{\prime}}(k+1)[\widehat{\alpha}(j-k)+\widehat{\gamma}] B(j, k+1) .
$$

But this leads to an infinite regress of $j$-dependence unless we assume $\widehat{\alpha}=0$, so we do this henceforth; this also kills the $j^{2}$ term in (A.50). Then $\boldsymbol{B}$ is a rescaled binomial, $B(n, k)=\left(\begin{array}{l}n \\ k\end{array}\right) \widehat{\gamma}^{n-k}\left(\widehat{\gamma}^{\prime}\right)^{k}$, and we have

$$
j B(j, k)=k B(j, k)+\frac{\widehat{\gamma}}{\widehat{\gamma}^{\prime}}(k+1) B(j, k+1) .
$$

Now insert (A.53) into (A.50). Normalizing to $\widehat{\gamma}^{\prime}=1$ and writing $\widehat{\gamma}=\xi$, then $\boldsymbol{B}$ becomes the $\xi$-binomial matrix $B_{\xi}$ defined in (4.6), and we obtain:

Corollary A.14. Let $\boldsymbol{A}=(A(n, k))_{0 \leqslant k \leqslant n}$ be a triangular array defined by the recurrence

$$
A(n, k)=\left(\beta_{n} k+\gamma_{n}\right) A(n-1, k)+\left(\beta_{n}^{\prime} k+\gamma_{n}^{\prime}\right) A(n-1, k-1)
$$

for $n \geqslant 1$, with initial condition $A(0, k)=\delta_{k 0}$. Then the matrix product $\boldsymbol{C}=\boldsymbol{A} B_{\xi}$ satisfies the recurrence

$$
\begin{gathered}
C(n, k)=\left[\left(\beta_{n}+2 \xi \beta_{n}^{\prime}\right) k+\gamma_{n}+\xi\left(\beta_{n}^{\prime}+\gamma_{n}^{\prime}\right)\right] C(n-1, k) \\
+\left(\beta_{n}^{\prime} k+\gamma_{n}^{\prime}\right) C(n-1, k-1) \\
+\xi\left(\beta_{n}+\xi \beta_{n}^{\prime}\right)(k+1) C(n-1, k+1)
\end{gathered}
$$

for $n \geqslant 1$, with initial condition $C(0, k)=\delta_{k 0}$. 
Specializing Corollary A.14 to the GKP case

$$
\beta_{n}=\beta, \quad \gamma_{n}=\alpha n+\gamma, \quad \beta_{n}^{\prime}=\beta^{\prime}, \quad \gamma_{n}^{\prime}=\alpha^{\prime} n+\gamma^{\prime},
$$

we get:

Corollary A.15. Let $\boldsymbol{A}=(A(n, k))_{0 \leqslant k \leqslant n}$ be a triangular array defined by the GKP recurrence

$$
A(n, k)=(\alpha n+\beta k+\gamma) A(n-1, k)+\left(\alpha^{\prime} n+\beta^{\prime} k+\gamma^{\prime}\right) A(n-1, k-1)
$$

for $n \geqslant 1$, with initial condition $A(0, k)=\delta_{k 0}$. Then the matrix product $\boldsymbol{C}=\boldsymbol{A} B_{\xi}$ satisfies the recurrence

$$
\begin{aligned}
C(n, k)=[ & \left.\left(\alpha+\xi \alpha^{\prime}\right) n+\left(\beta+2 \xi \beta^{\prime}\right) k+\gamma+\xi\left(\beta^{\prime}+\gamma^{\prime}\right)\right] C(n-1, k) \\
& +\left(\alpha^{\prime} n+\beta^{\prime} k+\gamma^{\prime}\right) C(n-1, k-1) \\
& +\xi\left(\beta+\xi \beta^{\prime}\right)(k+1) C(n-1, k+1)
\end{aligned}
$$

for $n \geqslant 1$, with initial condition $C(0, k)=\delta_{k 0}$.

The $\xi=1$ special case of this result was obtained by Spivey [52, Theorem 10]. In the special case $\xi=1$ and $\alpha^{\prime}=\beta^{\prime}=0$, a combinatorial proof was given by Mansour and Shattuck [39, Theorem 2.3].

Let us observe that the recurrence (A.58) is a special case of the Graham-KnuthPatashnik-Zhu recurrence (6.20). It reduces to a GKP recurrence in two cases: the trivial case $\xi=0$, and the case $\xi=-\beta / \beta^{\prime}$ corresponding to the Zhu involution (2.8). This suggests (but does not prove) that the matrix product $\boldsymbol{C}=\boldsymbol{T}(\boldsymbol{\mu}) B_{\xi}$ satisfies a GKP recurrence for generic parameters $\boldsymbol{\mu}$ only if $\xi=0$ or $\xi=-\beta / \beta^{\prime}$. We have verified, by a brute-force computation using $n=0,1,2,3$, that this is indeed the case: the only solutions to the equations $\boldsymbol{T}\left(\boldsymbol{\mu}^{\prime}\right)=\boldsymbol{T}(\boldsymbol{\mu}) B_{\xi}$ valid for generic parameters $\boldsymbol{\mu}$ are the identity map $\xi=0$ and the Zhu involution $\xi=-\beta / \beta^{\prime}$. Of course, there are additional solutions valid on subvarieties in $\boldsymbol{\mu}$-space; the goal of Problem 6.4 is to find them all.

The observation that (A.58) is a special case of the GKPZ recurrence suggests that we can go farther, and generalize Corollary A.15 by starting from a matrix $\boldsymbol{A}$ satisfying the GKPZ recurrence - or even more strongly, generalize Corollary A.14 by starting from a matrix $\boldsymbol{A}$ that satisfies an amalgamation of (A.54) and the GKPZ recurrence. This idea leads to the following:

Corollary A.16. Let $\boldsymbol{A}=(A(n, k))_{0 \leqslant k \leqslant n}$ be a triangular array defined by the recurrence

$$
\begin{aligned}
A(n, k) & =\left(\beta_{n} k+\gamma_{n}\right) A(n-1, k)+\left(\beta_{n}^{\prime} k+\gamma_{n}^{\prime}\right) A(n-1, k-1) \\
& +\sigma_{n}(n-k+1) A(n-1, k-2)+\tau_{n}(k+1) A(n-1, k+1)
\end{aligned}
$$


for $n \geqslant 1$, with initial condition $A(0, k)=\delta_{k 0}$. Then the matrix product $\boldsymbol{C}=\boldsymbol{A} B_{\xi}$ satisfies the recurrence

$$
\begin{aligned}
C(n, k)= & {\left[\left(\beta_{n}+2 \xi \beta_{n}^{\prime}-3 \xi^{2} \sigma_{n}\right) k+\gamma_{n}+\xi\left(\beta_{n}^{\prime}+\gamma_{n}^{\prime}\right)+\xi^{2} \sigma_{n}(n-1)\right] C(n-1, k) } \\
& +\left[\left(\beta_{n}^{\prime}-3 \xi \sigma_{n}\right) k+\gamma_{n}^{\prime}+\xi \sigma_{n}(2 n+1)\right] C(n-1, k-1) \\
& +\sigma_{n}(n-k+1) C(n-1, k-2) \\
& +\left(\tau_{n}+\xi \beta_{n}+\xi^{2} \beta_{n}^{\prime}-\xi^{3} \sigma_{n}\right)(k+1) C(n-1, k+1)
\end{aligned}
$$

for $n \geqslant 1$, with initial condition $C(0, k)=\delta_{k 0}$.

Proof. The matrix $\boldsymbol{C}=\boldsymbol{A} B_{\xi}$ satisfies

$$
\begin{aligned}
& C(n, k)=\sum_{j=0}^{n}\left(\beta_{n} j+\gamma_{n}\right) A(n-1, j) B_{\xi}(j, k)+\sum_{j=0}^{n}\left(\beta_{n}^{\prime} j+\gamma_{n}^{\prime}\right) A(n-1, j-1) B_{\xi}(j, k) \\
& +\sigma_{n} \sum_{j=0}^{n}(n-j+1) A(n-1, j-2) B_{\xi}(j, k)+\tau_{n} \sum_{j=0}^{n}(j+1) A(n-1, j+1) B_{\xi}(j, k)
\end{aligned}
$$

for $n \geqslant 1$. The first term on the right-hand side of (A.61) can be handled by using the identity

$$
j B_{\xi}(j, k)=k B_{\xi}(j, k)+\xi(k+1) B_{\xi}(j, k+1) .
$$

This yields

$$
\left(\beta_{n} k+\gamma_{n}\right) C(n-1, k)+\xi \beta_{n}(k+1) C(n-1, k+1) .
$$

The second term on the right-hand side of (A.61) can be dealt with by using the identities

$$
\begin{aligned}
B_{\xi}(j, k) & =B_{\xi}(j-1, k-1)+\xi B_{\xi}(j-1, k) \\
j B_{\xi}(j, k) & =k B_{\xi}(j-1, k-1)+(2 k+1) \xi B_{\xi}(j-1, k)+(k+1) \xi^{2} B_{\xi}(j-1, k+1)
\end{aligned}
$$

This yields

$$
\begin{aligned}
\xi\left(2 \beta_{n}^{\prime} k+\beta_{n}^{\prime}+\gamma_{n}^{\prime}\right) C(n-1, k)+\left(\beta_{n}^{\prime} k+\gamma_{n}^{\prime}\right) C & (n-1, k-1) \\
& +\xi^{2}(k+1) \beta_{n}^{\prime} C(n-1, k+1) .
\end{aligned}
$$

In the third term on the right-hand side of (A.61), we use the identities

$$
\begin{aligned}
B_{\xi}(j, k)= & B_{\xi}(j-2, k-2)+2 \xi B_{\xi}(j-2, k-1)+\xi^{2} B_{\xi}(j-2, k) \\
j B_{\xi}(j, k)= & k B_{\xi}(j-2, k-2)+(3 k+1) \xi B_{\xi}(j-2, k-1) \\
& \quad+(3 k+2) \xi^{2} B_{\xi}(j-2, k)+(k+1) \xi^{3} B_{\xi}(j-2, k+1)
\end{aligned}
$$


This yields

$$
\begin{aligned}
\sigma_{n}\left[\xi^{2}(n-3 k-1)\right. & C(n-1, k)+\xi(2 n-3 k+1) C(n-1, k-1) \\
& \left.+(n-k+1) C(n-1, k-2)-\xi^{3}(k+1) C(n-1, k+1)\right] .
\end{aligned}
$$

And finally, the fourth term on the right-hand side of (A.61) is handled by using the absorption/extraction identity $(j+1) B_{\xi}(j, k)=(k+1) B_{\xi}(j+1, k+1)$, yielding

$$
\tau_{n}(k+1) C(n-1, k+1) .
$$

Putting together the partial results (A.63)/(A.65)/(A.67)/(A.68), we arrive at the claimed result (A.60).

Specializing Corollary A.16 to the GKPZ case

$$
\beta_{n}=\beta, \quad \gamma_{n}=\alpha n+\gamma, \quad \beta_{n}^{\prime}=\beta^{\prime}, \quad \gamma_{n}^{\prime}=\alpha^{\prime} n+\gamma^{\prime}, \quad \sigma_{n}=\sigma, \quad \tau_{n}=\tau,
$$

we get:

Corollary A.17. Let $\boldsymbol{A}=(A(n, k))_{0 \leqslant k \leqslant n}$ be a triangular array defined by the GKPZ recurrence

$$
\begin{array}{r}
A(n, k)=(\alpha n+\beta k+\gamma) A(n-1, k)+\left(\alpha^{\prime} n+\beta^{\prime} k+\gamma^{\prime}\right) A(n-1, k-1) \\
+\sigma(n-k+1) A(n-1, k-2)+\tau(k+1) A(n-1, k+1)
\end{array}
$$

for $n \geqslant 1$, with initial condition $A(0, k)=\delta_{k 0}$. Then the matrix product $\boldsymbol{C}=\boldsymbol{A} B_{\xi}$ satisfies the GKPZ recurrence

$$
\begin{aligned}
C(n, k)= & {\left[\left(\alpha+\xi^{2} \sigma\right) n+\left(\beta+2 \xi \beta^{\prime}-3 \xi^{2} \sigma\right) k+\gamma+\xi\left(\beta^{\prime}+\gamma^{\prime}\right)-\xi^{2} \sigma\right] C(n-1, k) } \\
& +\left[\left(\alpha^{\prime}+2 \xi \sigma\right) n+\left(\beta^{\prime}-3 \xi \sigma\right) k+\gamma^{\prime}+\xi \sigma\right] C(n-1, k-1) \\
& +\sigma(n-k+1) C(n-1, k-2) \\
& +\left(\tau_{n}+\xi \beta+\xi^{2} \beta^{\prime}-\xi^{3} \sigma\right)(k+1) C(n-1, k+1)
\end{aligned}
$$

for $n \geqslant 1$, with initial condition $C(0, k)=\delta_{k 0}$.

\section{B Inverse pairs of lower-triangular arrays}

Let $A=\left(a_{n k}\right)_{n \geqslant k \geqslant 0}$ be a lower-triangular array with entries in a commutative ring $R$, and define as usual the row-generating polynomials

$$
A_{n}(x)=\sum_{k=0}^{n} a_{n k} x^{k} \in R[x]
$$

and the reversed row-generating polynomials

$$
\bar{A}_{n}(x)=x^{n} A_{n}(1 / x)=\sum_{k=0}^{n} a_{n k} x^{n-k} \in R[x] .
$$


Lemma B.1 (Inverse pairs of lower-triangular arrays). Let $A=\left(a_{n k}\right)_{n \geqslant k \geqslant 0}$ and $B=$ $\left(b_{n k}\right)_{n \geqslant k \geqslant 0}$ be lower-triangular arrays with entries in a commutative ring $R$, and let $A_{n}(x)$, $\bar{A}_{n}(x)$ and $B_{n}(x), \bar{B}_{n}(x)$ be their row-generating polynomials. Let $\alpha \in R$. Then the following are equivalent:

(a) $A_{n}(x)=(1+\alpha x)^{n} B_{n}\left(\frac{x}{1+\alpha x}\right)$.

(b) $B_{n}(x)=(1-\alpha x)^{n} A_{n}\left(\frac{x}{1-\alpha x}\right)$.

(c) $\bar{A}_{n}(x)=\bar{B}_{n}(x+\alpha)$.

(d) $\bar{B}_{n}(x)=\bar{A}_{n}(x-\alpha)$.

(e) $a_{n k}=\sum_{j=0}^{k} \alpha^{k-j}\left(\begin{array}{l}n-j \\ k-j\end{array}\right) b_{n j}$.

(f) $b_{n k}=\sum_{j=0}^{k}(-\alpha)^{k-j}\left(\begin{array}{l}n-j \\ k-j\end{array}\right) a_{n j}$.

(g) $a_{n, n-k}=\sum_{j=0}^{k} b_{n, n-j}\left(\begin{array}{l}j \\ k\end{array}\right) \alpha^{j-k}$.

(h) $b_{n, n-k}=\sum_{j=0}^{k} a_{n, n-j}\left(\begin{array}{l}j \\ k\end{array}\right)(-\alpha)^{j-k}$.

Proof. The equivalence of (a)-(d) is an easy manipulation of generating functions; extracting the coefficient of $x^{k}$ yields (e)-(h).

Pairs $(A, B)$ satisfying the conditions of Lemma B.1 arise frequently in combinatorial applications, most often with $\alpha=1$.

Let us remark that $(1+\alpha x)^{n} B_{n}\left(\frac{x}{1+\alpha x}\right)$ is here simply an abbreviation for $\sum_{k=0}^{n} b_{n k} x^{k}(1+\alpha x)^{n-k}$. So there is no need to work in a field of rational functions; everything can be done in the ring $R[x]$ of polynomials, and moreover the ring $R$ is not required to be an integral domain.

Let us also observe that, in Lemma B.1, the labels $n$ "go for the ride": the statements for different $n$ are completely unrelated. So we can just rename $A_{n}, B_{n}$ as $f, g$. Furthermore, in statements (a,b,e,f), $f$ and $g$ need not be polynomials; they can be general formal power series. And the power $n$ in $(1 \pm \alpha x)^{n}$ need not be a positive integer (provided that the ring $R$ contains the rationals); it can be an indeterminate, call it $p$. So what $(\mathrm{a}) \Longleftrightarrow$ (b) really asserts is the easily verified equivalence

$$
f(x)=(1+\alpha x)^{p} g\left(\frac{x}{1+\alpha x}\right) \quad \Longleftrightarrow \quad g(x)=(1-\alpha x)^{p} f\left(\frac{x}{1-\alpha x}\right),
$$


in which the coefficient of each power of $x$ is a polynomial (with coefficients in $R \supseteq \mathbb{Q}$ ) in the indeterminates $p$ and $\alpha$. Similarly, (e) $\Longleftrightarrow(\mathrm{f})$ is the binomial identity

$$
\sum_{j=0}^{k} \alpha^{k-j}\left(\begin{array}{l}
p-j \\
k-j
\end{array}\right)(-\alpha)^{j-\ell}\left(\begin{array}{l}
p-\ell \\
j-\ell
\end{array}\right)=\delta_{k \ell}
$$

in which both sides are polynomials (with coefficients in $\mathbb{Q}$ ) in the indeterminates $p$ and $\alpha$. The identity (B.4) can be found in Riordan [44, p. 49, Table 2.1, item 3]. ${ }^{15}$ It can also be proven without using generating functions: First rewrite the Chu-Vandermonde identity $\sum_{i=0}^{m}\left(\begin{array}{c}-(x+1) \\ i\end{array}\right)\left(\begin{array}{c}x+y+1 \\ m-i\end{array}\right)=\left(\begin{array}{c}y \\ m\end{array}\right)$ as $\sum_{i=0}^{m}(-1)^{i}\left(\begin{array}{c}x+i \\ i\end{array}\right)\left(\begin{array}{c}x+y+1 \\ m-i\end{array}\right)=\left(\begin{array}{c}y \\ m\end{array}\right)$. Specializing to $y=m-1$ we have $\sum_{i=0}^{m}(-1)^{i}\left(\begin{array}{c}x+i \\ i\end{array}\right)\left(\begin{array}{c}x+m \\ m-i\end{array}\right)=\left(\begin{array}{c}m-1 \\ m\end{array}\right)=\delta_{m 0} \cdot{ }^{16}$ Substitute $m=k-\ell$ and $x=p-k$, and then rewrite the summation in terms of $j=k-i$ : this yields (B.4).

\section{References}

[1] M. Aigner, Catalan and other numbers: a recurrent theme, in: Algebraic Combinatorics and Computer Science, edited by H. Crapo and D. Senato (Springer-Verlag Italia, Milan, 2001), pp. 347-390.

[2] J.F. Barbero G., J. Salas, and E.J.S. Villaseñor, Bivariate generating functions for a class of linear recurrences: General structure, J. Combin. Theory A 125, 146-165 (2014), arXiv:1307.2010 [math.CO].

[3] J.F. Barbero G., J. Salas, and E.J.S. Villaseñor. Generalized Stirling permutations and forests: Higher-order Eulerian and Ward numbers, Electron. J. Combin. 22, \#P3.37 (2015), arXiv: 1307.5624 [math.CO].

[4] P. Barry, Continued fractions and transformations of integer sequences, J. Integer Seq. 12, article 09.7.6 (2009).

[5] P. Barry, Riordan Arrays: A Primer (Logic Press, County Kildare, Ireland, 2016).

[6] P. Barry, The $1 / k$-Eulerian polynomials as moments, via exponential Riordan arrays, preprint (March 2018), arXiv:1803.10308 [math.CO].

[7] F. Brenti, Combinatorics and total positivity, J. Combin. Theory A 71, 175-218 (1995).

\footnotetext{
${ }^{15}$ However, his $\left(\begin{array}{c}p-k \\ p-n\end{array}\right)$ should be rewritten as $\left(\begin{array}{c}p-k \\ n-k\end{array}\right)$ in order to bring out more clearly that $p$ can be an indeterminate.

${ }^{16}$ We have not been able to find either of these two latter identities in any of the standard tables of binomial identities, such as Gould's [33, Table 3]. But an essentially equivalent identity can be found in Riordan [44, p. 8, eq. (5)], and all such identities can in any case be considered as simple rephrasings and/or specializations of $\mathrm{Chu}-$ Vandermonde.
} 
[8] F. Brenti, A class of $q$-symmetric functions arising from plethysm, J. Combin. Theory A 91, 137-170 (2000).

[9] L. Carlitz, Eulerian numbers and polynomials of higher order, Duke Math. J. 27, 401-423 (1960).

[10] L. Carlitz and R. Scoville, Some permutation problems, J. Combin. Theory A 22, 129-145 (1977).

[11] W.Y.C. Chen, L.X.W. Wang and A.L.B. Yang, Recurrence relations for strongly q-log-convex polynomials, Canad. Math. Bull. 54, 217-229 (2011).

[12] P. Di Francesco and R. Kedem, $Q$-systems, heaps, paths and cluster positivity, Commun. Math. Phys. 293, 727-802 (2010).

[13] J.F. Dillon and J.P. Roselle, Eulerian numbers of higher order, Duke Math. J. 35, 247-256 (1968).

[14] T. Dokchitser, web page "Group Names", https://groupnames.org/ = https:// people.maths.bris.ac.uk/ matyd/

[15] D. Dumont, Pics de cycle et dérivées partielles, Séminaire Lotharingien de Combinatoire 13, article B13a (1986).

[16] D. Dumont, A continued fraction for Stirling numbers of the second kind, unpublished note (1989), cited in [66].

[17] D. Dumont, Further triangles of Seidel-Arnold type and continued fractions related to Euler and Springer numbers, Adv. Appl. Math. 16, 275-296 (1995).

[18] D. Dumont and J. Zeng, Further results on the Euler and Genocchi numbers, Aequationes Math. 47, 31-42 (1994).

[19] A. Elvey Price and A.D. Sokal, Phylogenetic trees, augmented perfect matchings, and a Thron-type continued fraction (T-fraction) for the Ward polynomials, preprint (March 2020), arXiv:2001.01468v2 [math.CO].

[20] G. Eneström, Die Schriften Eulers chronologisch nach den Jahren geordnet, in denen sie verfaßt worden sind, Jahresbericht der Deutschen Mathematiker-Vereinigung (Teubner, Leipzig, 1913).

[21] L. Euler, De transformatione serierum, Part II, Chapter 1 of Institutiones Calculi Differentialis cum eius Usu in Analysi Finitorum ac Doctrina Serierum [Foundations of Differential Calculus, with Applications to Finite Analysis and Series], 1755; reprinted in Opera Omnia, ser. 1, vol. 10. [Latin original available at http://eulerarchive.maa.org/pages/E212.html; English translation available at https://www.agtz.mathematik.uni-mainz.de/ algebraische-geometrie/van-straten/euler-kreis-mainz/]

[22] L. Euler, Methodus summandi superior ulterius promota, Part II, Chapter 7 of Institutiones Calculi Differentialis cum eius Usu in Analysi Finitorum ac Doctrina Serierum [Foundations of Differential Calculus, with Applications to Finite Analysis and Series], 1755; reprinted in Opera Omnia, ser. 1, vol. 14, pp. 124137. [Latin original available at http://eulerarchive.maa.org/pages/E055.html; 
English translation available at https://www.agtz.mathematik.uni-mainz.de/ algebraische-geometrie/van-straten/euler-kreis-mainz/]

[23] L. Euler, De seriebus divergentibus, Novi Commentarii Academiae Scientiarum Petropolitanae 5, 205-237 (1760). [Latin original and English and German translations available at http://eulerarchive.maa.org/pages/E247.html]

[24] L. Euler, De transformatione seriei divergentis $1-m x+m(m+n) x^{2}-$ $m(m+n)(m+2 n) x^{3}+$ etc. in fractionem continuam, Nova Acta Academiae Scientarum Imperialis Petropolitanae 2, 36-45 (1788); reprinted in Opera Omnia, ser. 1, vol. 16, pp. 34-46. [Latin original and English and German translations available at http://eulerarchive.maa.org/pages/E616.html]

[25] S.M. Fallat and C.R. Johnson, Totally Nonnegative Matrices (Princeton University Press, Princeton NJ, 2011).

[26] A.E. Fekete, A propos "Two notes on notation", Amer. Math. Monthly 101, 771-778 (1994).

[27] P. Flajolet, Combinatorial aspects of continued fractions, Discrete Math. 32, 125-161 (1980).

[28] P. Flajolet, On congruences and continued fractions for some classical combinatorial quantities, Discrete Math. 41, 145-153 (1982).

[29] D. Foata and M.-P. Schützenberger, Théorie Géométrique des Polynômes Eulériens, Lecture Notes in Mathematics \#138 (Springer-Verlag, Berlin-New York, 1970). Available on-line at http://www.emis.de/journals/SLC/books/foaschuetz.html

[30] G. Frobenius, Über die Bernoullischen Zahlen und die Eulerschen Polynome, Sitzungsberichte der Königlich Preussischen Akademie der Wissenschaften [Berlin] (1910), 809-847.

[31] F.R. Gantmacher and M.G. Krein, Oscillation Matrices and Kernels and Small Vibrations of Mechanical Systems (AMS Chelsea Publishing, Providence RI, 2002). Based on the second Russian edition, 1950.

[32] F. Gantmakher and M. Krein, Sur les matrices complètement non négatives et oscillatoires, Compositio Math. 4, 445-476 (1937).

[33] H.W. Gould, Combinatorial Identities (Henry W. Gould, Morgantown, W.Va., 1972).

[34] R.L. Graham, D.E. Knuth and O. Patashnik, Concrete Mathematics: A Foundation for Computer Science, 2nd ed. (Addison-Wesley, Reading, MA, 1994).

[35] S. Karlin, Total Positivity (Stanford University Press, Stanford CA, 1968).

[36] G. Ksavrelof and J. Zeng, Two involutions for signed excedance numbers, Séminaire Lotharingien de Combinatoire 49, article B49e (2003).

[37] L.L. Liu and Y. Wang, On the log-convexity of combinatorial sequences, Adv. Appl. Math. 39, 453-476 (2007).

[38] S.-M. Ma, Counting permutations by numbers of excedances, fixed points and cycles, Bull. Austral. Math Soc. 85, 415-421 (2012). 
[39] T. Mansour and M. Shattuck, A combinatorial approach to a general two-term recurrence, Discrete Appl. Math. 161, 2084-2094 (2013).

[40] E. Neuwirth, Recursively defined combinatorial functions: extending Galton's boards, Discrete Math. 132, 33-51 (2001).

[41] The On-Line Encyclopedia of Integer Sequences, published electronically at http: //oeis.org

[42] M. Pétréolle, A.D. Sokal and B.-X. Zhu, Lattice paths and branched continued fractions: An infinite sequence of generalizations of the Stieltjes-Rogers and ThronRogers polynomials, with coefficientwise Hankel-total positivity, preprint (July 2018), arXiv:1807.03271 [math.CO], to appear in Memoirs of the American Mathematical Society.

[43] A. Pinkus, Totally Positive Matrices (Cambridge University Press, Cambridge, 2010).

[44] J. Riordan, Combinatorial Identities (Wiley, New York, 1968).

[45] E. Roblet and X.G. Viennot, Théorie combinatoire des T-fractions et approximants de Padé en deux points, Discrete Math. 153, 271-288 (1996).

[46] J. Salas and A.D. Sokal, unpublished (2018).

[47] C.D. Savage and G. Viswanathan, The 1/k-Eulerian polynomials, Electron. J. Combin. 19, no. 1, \#P9 (2012).

[48] A.D. Sokal, Coefficientwise total positivity (via continued fractions) for some Hankel matrices of combinatorial polynomials, talk at the Séminaire de Combinatoire Philippe Flajolet, Institut Henri Poincaré, Paris, 5 June 2014; transparencies available at http://semflajolet.math.cnrs.fr/index.php/Main/2013-2014

[49] A.D. Sokal, Coefficientwise total positivity (via continued fractions) for some Hankel matrices of combinatorial polynomials, in preparation.

[50] A.D. Sokal, unpublished (2014).

[51] A.D. Sokal and J. Zeng, Some multivariate master polynomials for permutations, set partitions, and perfect matchings, and their continued fractions, preprint (March 2020), arXiv:2003.08192 [math.CO].

[52] M.Z. Spivey, On solutions to a general combinatorial recurrence, J. Integer Seq. 14, article 11.9 .7 (2011).

[53] M.Z. Spivey, The binomial recurrence, Math. Mag. 89, 192-195 (2016).

[54] M.Z. Spivey, Recurrent combinatorial sums and binomial-type theorems, J. Integer Seq. 23, article 20.5.5 (2020).

[55] R.P. Stanley, Enumerative Combinatorics, vol. 1 (Wadsworth \& Brooks/Cole, Monterey, California, 1986). Reprinted by Cambridge University Press, 1999.

[56] T.J. Stieltjes, Recherches sur les fractions continues, Ann. Fac. Sci. Toulouse 8, J1J122 (1894) and 9, A1-A47 (1895). [Reprinted, together with an English translation, in T.J. Stieltjes, Euvres Complètes/Collected Papers (Springer-Verlag, Berlin, 1993), vol. II, pp. 401-566 and 609-745.] 
[57] P. Théorêt, Hyperbinomiales: Doubles suites satisfaisant à des équations aux différences partielles de dimension et d'ordre deux de la forme $H(n, k)=$ $p(n, k) H(n-1, k)+q(n, k) H(n-1, k-1)$, Thèse de doctorat, Université du Québec à Montréal (1994).

[58] P. Théorêt, Fonctions génératrices pour une classe d'équations aux différences partielles, Ann. Sci. Math. Québec 19, 91-105 (1995).

[59] P. Théorêt, Relations matricielles pour hyperbinomiales, Ann. Sci. Math. Québec 19, 197-212 (1995).

[60] W.J. Thron, Some properties of continued fractions $1+d_{0} z+K\left(z /\left(1+d_{n} z\right)\right)$, Bull. Amer. Math. Soc. 54, 206-218 (1948).

[61] G. Viennot, Une théorie combinatoire des polynômes orthogonaux généraux, Notes de conférences données à l'Université du Québec à Montréal, septembre-octobre 1983. Available on-line at http://www.xavierviennot.org/xavier/polynomes_ orthogonaux.html

[62] G. Viennot, A combinatorial theory for general orthogonal polynomials with extensions and applications, in Polynômes Orthogonaux et Applications (Lecture Notes in Mathematics \#1171), edited by C. Brezinski et al. (Springer-Verlag, Berlin, 1985), pp. 139-157.

[63] H.S. Wall, Analytic Theory of Continued Fractions (Van Nostrand, New York, 1948).

[64] H.S. Wilf, The method of characteristics, and 'problem 89' of Graham, Knuth and Patashnik, preprint 2004, arXiv:math/0406620.

[65] J. Zeng, Énumérations de permutations et J-fractions continues, European J. Combin. 14, 373-382 (1993).

[66] J. Zeng, The $q$-Stirling numbers, continued fractions and the $q$-Charlier and $q$ Laguerre polynomials, J. Comput. Appl. Math. 57, 413-424 (1995).

[67] B.-X. Zhu, unpublished manuscript (2018).

[68] B.-X. Zhu, A generalized Eulerian triangle from staircase tableaux and tree-like tableaux, J. Combin. Theory A 172, 105206 (2020). 